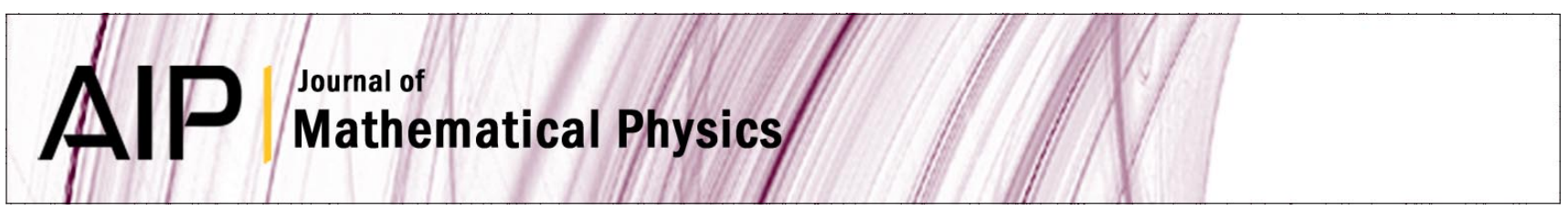

\title{
Harmonic sums and polylogarithms generated by cyclotomic polynomials
}

Jakob Ablinger, Johannes Blümlein, and Carsten Schneider

Citation: J. Math. Phys. 52, 102301 (2011); doi: 10.1063/1.3629472

View online: http://dx.doi.org/10.1063/1.3629472

View Table of Contents: http://jmp.aip.org/resource/1/JMAPAQ/v52/i10

Published by the American Institute of Physics.

\section{Related Articles}

The Casimir spectrum revisited

J. Math. Phys. 52, 072103 (2011)

Renormalized interaction of relativistic bosons with delta function potentials

J. Math. Phys. 51, 082305 (2010)

Callan-Symanzik equation and asymptotic freedom in the Marr-Shimamoto model

J. Math. Phys. 51, 052107 (2010)

Quasiperiodic motions in dynamical systems: Review of a renormalization group approach

J. Math. Phys. 51, 015207 (2010)

Nonperturbative one-loop effective action for electrodynamics in curved space-time

J. Math. Phys. 50, 102301 (2009)

\section{Additional information on J. Math. Phys.}

Journal Homepage: http://jmp.aip.org/

Journal Information: http://jmp.aip.org/about/about_the_journal

Top downloads: http://jmp.aip.org/features/most_downloaded

Information for Authors: http://jmp.aip.org/authors

\section{ADVERTISEMENT}

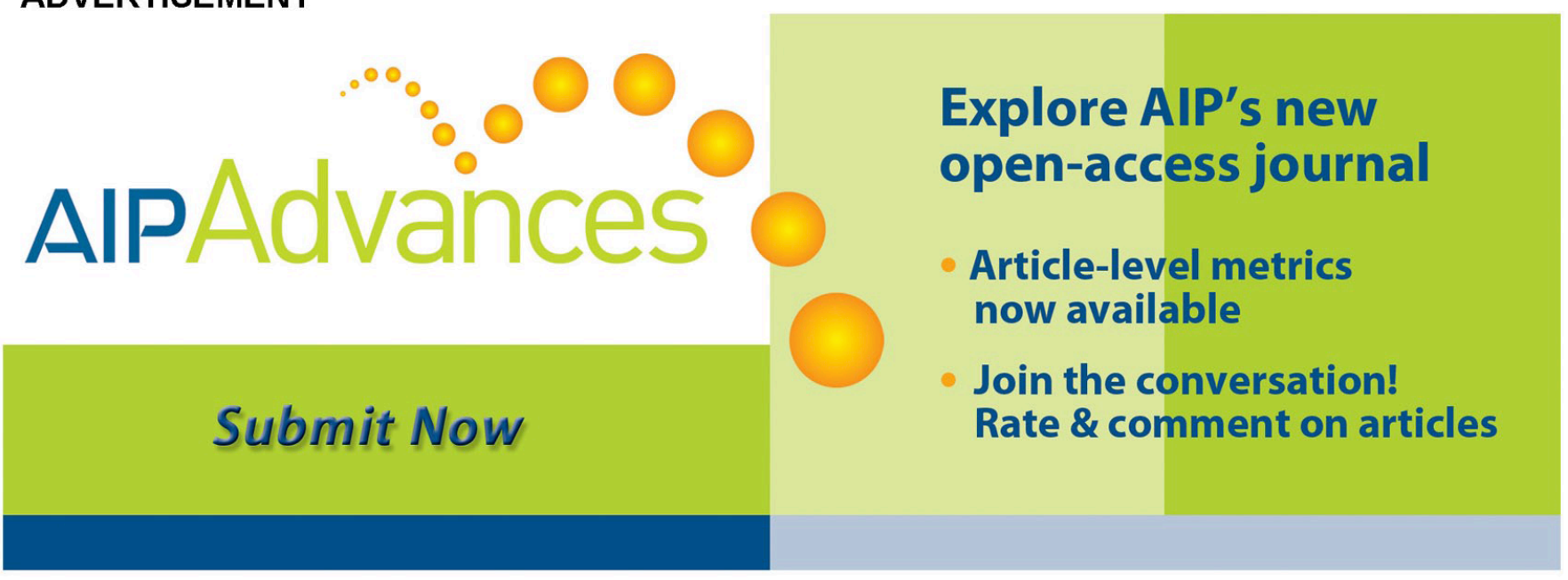




\title{
Harmonic sums and polylogarithms generated by cyclotomic polynomials
}

\author{
Jakob Ablinger, ${ }^{1}$ Johannes Blümlein, ${ }^{2, a)}$ and Carsten Schneider ${ }^{1}$ \\ ${ }^{1}$ Research Institute for Symbolic Computation (RISC), Johannes Kepler University, \\ Altenbergerstraße 69, A-4040, Linz, Austria \\ ${ }^{2}$ Deutsches Elektronen-Synchrotron, DESY, Platanenallee 6, D-15738 Zeuthen, Germany
}

(Received 30 May 2011; accepted 3 August 2011; published online 3 October 2011)

\begin{abstract}
The computation of Feynman integrals in massive higher order perturbative calculations in renormalizable quantum field theories requires extensions of multiply nested harmonic sums, which can be generated as real representations by Mellin transforms of Poincaré-iterated integrals, including denominators of higher cyclotomic polynomials. We derive the cyclotomic harmonic polylogarithms and harmonic sums and study their algebraic and structural relations. The analytic continuation of cyclotomic harmonic sums to complex values of $N$ is performed using analytic representations. We also consider special values of the cyclotomic harmonic polylogarithms at argument $x=1$, respectively, for the cyclotomic harmonic sums at $N \rightarrow \infty$, which are related to colored multiple zeta values, deriving various of their relations, based on the stuffle and shuffle algebras and three multiple argument relations. We also consider infinite generalized nested harmonic sums at roots of unity which are related to the infinite cyclotomic harmonic sums. Basis representations are derived for weight $\mathbf{w}=1,2$ sums up to cyclotomy $I=20$. This paper is dedicated to Martinus Veltman on the occasion of his 80th birthday. (C) 2011 American Institute of Physics. [doi:10.1063/1.3629472]
\end{abstract}

\section{INTRODUCTION}

The analytic calculation of Feynman integrals requires the complete understanding of the associated mathematical structures at a given loop level. By the pioneering work in Refs. 1 and 2 this has been thoroughly achieved for one loop integral occurring in renormalizable quantum field theories. The corresponding complete framework in case of higher order calculations is, however, not yet available. Which mathematical functions are of relevance there is revealed stepwise in specific higher order calculations. Here, higher transcendental functions, such as the generalized hypergeometric functions and their generalizations, ${ }^{3}$ play a central role. Their series expansion in the dimensional parameter ${ }^{1,4}$ leads to nested infinite sums over products of digamma functions, ${ }^{5}$ cf. Ref. 6 . The nested harmonic sums ${ }^{7,8}$ are defined by

$$
S_{b, \vec{a}}(N)=\sum_{k=1}^{N} \frac{(\operatorname{sign}(b))^{k}}{k^{|b|}} S_{\vec{a}}(k), \quad S_{\emptyset}(N)=1, \quad b, a_{i} \in \mathbb{Z} \backslash\{0\} .
$$

They form a quasi-shuffle algebra. ${ }^{9}$ Their values for $N \rightarrow \infty$ are the multiple zeta values $\zeta_{\vec{a}}$, respectively, Euler-Zagier values ${ }^{10}$ defined by

$$
\zeta_{b, \vec{a}}=\lim _{N \rightarrow \infty} S_{b, \vec{a}}(N), \quad b \neq 1,
$$

see Ref. 11. In case of massless problems to 3-loop order the results for single scale quantities in Mellin space can be written by polynomial expressions in terms of $S_{\vec{a}}(N)$ and $\zeta_{\vec{a}}$ with coefficients

a) Author to whom correspondence should be addressed. Electronic mail: Johannes.Bluemlein@ desy.de. 
being from the rational function field $\mathbb{Q}(N)$, cf., e.g., Ref. 12. In this context, we consider the Mellin transform

$$
\mathbf{M}[f(x)](N)=\int_{0}^{1} d x x^{N} f(x) .
$$

In most of the applications below we assume $N \in \mathbb{N}_{+}=\mathbb{N} \backslash\{0\}$. In computations at even higher orders in the coupling constant and through finite mass effects, however, generalizations of the nested harmonic sums contribute, at least in intermediary results. One extension concerns the socalled generalized harmonic sums ${ }^{13,14}$ given by

$$
S_{b, \vec{a}}(\zeta, \vec{\xi} ; N)=\sum_{k=1}^{N} \frac{\zeta^{k}}{k^{b}} S_{\vec{\xi}}(\vec{r} ; k)
$$

with $b, a_{i} \in \mathbb{N}_{+} ; \zeta, \xi_{i} \in \mathbb{R}^{*}=\mathbb{R} \backslash\{0\}$. Known examples are related to the second index set $\xi_{i}$ $\in\{1,-1,1 / 2,-1 / 2,2,-2\}$, cf. Refs. 12,15 , and 16 .

In case of single scale, problems with two massive lines and $m_{1}=m_{2}$ at 3-loop order summation are also required over terms

$$
\frac{( \pm 1)^{k}}{(2 k+1)^{n}}
$$

which is a special case of

$$
\frac{( \pm 1)^{k}}{(l \cdot k+m)^{n}}
$$

with $l, m, n \in \mathbb{N}_{+}$. Note that we have deliberately chosen a real representation here, which is of practical importance in case of fast polynomial operations needed for solving nested summation problems. ${ }^{17,87}$ Sums containing fractional terms $m / l$ were considered in the context of colored harmonic sums in Refs. 20-22. It is expected that sums of this kind and their iterations will occur in a wide class of massive calculations in higher order loop calculations in quantum electrodynamics, quantum chromodynamics, and other renormalizable quantum field theories, in particular, studying single distributions, but also for more variable differential distributions. Usually, objects of this kind emerge first at intermediary steps and, at even higher orders, they occur in the final results. Therefore, these quantities have to be understood and methods have to be provided to perform these sums. This does not apply to integer values of the Mellin variable $N$ only, but also to the analytic continuation of these quantities to $N \in \mathbb{C}$, since the experimental applications require the Mellin inversion into momentum-fraction space.

We show that the single sums (1.5) and their nested iterations can be obtained from linear combinations of Mellin transforms of harmonic polylogarithms over an alphabet of letters containing $x^{l} / \Phi_{k}(x), 0<l<\operatorname{deg}\left(\Phi_{k}(x)\right)$, where $\Phi_{k}(x)$ denotes the $k$ th cyclotomic polynomial. ${ }^{23}$ One may form words by Poincaré-iterated integrals ${ }^{25}$ over this alphabet, which leads to the cyclotomic harmonic polylogarithms $\mathfrak{H}$, forming a shuffle algebra. This class extends the harmonic polylogarithms. ${ }^{26}$ The Mellin transform of elements of $\mathfrak{H}$ has support $x \in[0,1]$, but requires an extension in the Mellin variable $N \in \mathbb{N}$,

$$
N \rightarrow k \cdot N,
$$

where $k$ denotes the index of $\Phi_{k}(x)$. This assumption allows to associate nested harmonic sums of the cyclotomic type by this Mellin transform. Special values are obtained by either the cyclotomic harmonic polylogarithms $H_{\vec{a}}(x)$ at $x=1$ or the associated nested harmonic sums for $N \rightarrow \infty$. They extend the multiple zeta values and Euler-Zagier values, cf. Sec. V In the present paper, we investigate relations and representations of these three classes of quantities. Special emphasis has been put on the class of nested sums (1.4), where all derived algorithms have been incorporated within the computer algebra package HARMONICSUMS. ${ }^{27}$

The paper is organized as follows. In Sec. II we establish the connection between the cyclotomic harmonic sums and the cyclotomic harmonic polylogarithms through the Mellin transform, 
at modified argument $k N$. The basic properties of the cyclotomic harmonic polylogarithms are investigated in Sec. III. The cyclotomic harmonic polylogarithms obey a shuffle algebra. The nested sums at finite values of $N$ are studied in Sec. IV, including their algebraic and structural relations, generalizing Refs. 28-31. Here are also three multiple argument relations of interest. The analytic continuation of the new harmonic sums to complex values of $N$ is presented based on recursion and asymptotic representations, similar to the case of the nested harmonic sum. ${ }^{29-32}$ The representation of the cyclotomic harmonic sums requires to know their values at $N \rightarrow \infty$, which are equivalently given by the cyclotomic harmonic polylogarithms at $x=1$. The set of special numbers spanning the Euler-Zagier and multiple zeta values ${ }^{11}$ is extended. We study the cases of weight $\mathrm{W}=1,2$ up to cyclotomy $\mathrm{I}=20$ and derive the corresponding relations based on the stuffle and shuffle algebra and three multiple argument relations. Furthermore, we investigate the relations of the cyclotomic harmonic sums for $N \rightarrow \infty$ extending for words resulting from the alphabet $( \pm 1)^{k} / k,( \pm 1)^{k} /(2 k+1)$, cf. Sec. V. For the cyclotomic harmonic polylogarithms, harmonic sums, and their values at $N \rightarrow \infty$, basis representations are derived. In Sec. VI we study the relations of the infinite nested harmonic sums with numerators at the $l$ th root of unity, $I \leq 20$ for weight $\mathbf{w}=1,2$. Here, also the distribution relation, cf. Ref. 19, is considered beyond the relations mentioned before. We also consider a relation valid for finite generalized harmonic sums with root numerator weights. Section VII contains the conclusions. Some technical details are given in the Appendices.

\section{BASIC FORMALISM}

We consider cyclotomic harmonic sums defined by

$$
S_{\left\{a_{1}, b_{1}, c_{1}\right\}, \ldots,\left\{a_{l}, b_{l}, c_{l}\right\}}\left(s_{1}, \ldots, s_{l} ; N\right)=\sum_{k_{1}=1}^{N} \frac{s_{1}^{k}}{\left(a_{1} k_{1}+b_{1}\right)^{c_{1}}} S_{\left\{a_{2}, b_{2}, c_{2}\right\} ; \ldots ;\left\{a_{l}, b_{l}, c_{l}\right\}}\left(s_{2}, \ldots, s_{l} ; k_{1}\right), \quad S_{\emptyset}=1,
$$

where $a_{i}, c_{i} \in \mathbb{N}_{+}, b_{i} \in \mathbb{N}, s_{i}= \pm 1, a_{i}>b_{i}$; the weight of this sum is defined by $c_{1}+\cdots+c_{l}$ and $\left\{a_{i}, b_{i}, c_{i}\right\}$ denote lists, not sets. One may generalize this case further allowing $s_{i} \in \mathbb{R}^{*} .{ }^{14}$ Of special interest will be the infinite cyclotomic harmonic polylogarithms defined by

$$
\sigma_{\left\{a_{1}, b_{1}, c_{1}\right\}, \ldots,\left\{a_{l}, b_{l}, c_{l}\right\}}\left(s_{1}, \ldots, s_{l}\right)=\lim _{N \rightarrow \infty} S_{\left\{a_{1}, b_{1}, c_{1}\right\}, \ldots,\left\{a_{l}, b_{l}, c_{l}\right\}}\left(s_{1}, \ldots, s_{l} ; N\right)
$$

which diverge if $c_{1}=1, s_{1}=1$. Sometimes, we will use the notation

$$
\begin{gathered}
S_{\left\{a_{1}, b_{1}, c_{1}\right\}, \ldots,\left\{a_{l}, b_{l}, c_{l}\right\}}\left(s_{1}, \ldots, s_{l} ; N\right)=S_{\left\{a_{1}, b_{1}, s_{1} c_{1}\right\}, \ldots,\left\{a_{l}, b_{l}, s_{l} c_{l}\right\}}(N), \\
\sigma_{\left\{a_{1}, b_{1}, c_{1}\right\}, \ldots,\left\{a_{l}, b_{l}, c_{l}\right\}}\left(s_{1}, \ldots, s_{l}\right)=\sigma_{\left\{a_{1}, b_{1}, s_{1} c_{1}\right\}, \ldots,\left\{a_{l}, b_{l}, s_{l} c_{l}\right\}}
\end{gathered}
$$

as a shortcut below.

For further considerations, we rely on the following procedure which transforms the sums to integrals. The denominators have the following integral representation:

$$
\begin{gathered}
\frac{( \pm 1)^{k}}{a k+b}=\int_{0}^{1} d x x^{a k+b-1}( \pm 1)^{k} \\
\frac{( \pm 1)^{k}}{(a k+b)^{c}}=\int_{0}^{1} \frac{d x_{1}}{x_{1}} \int_{0}^{x_{1}} \frac{d x_{2}}{x_{2}} \ldots \int_{0}^{x_{c-2}} \frac{d x_{c-1}}{x_{c-1}} \int_{0}^{x_{c-1}} d x_{c} x_{c}^{a k+b-1}( \pm 1)^{k},
\end{gathered}
$$

and the sum over $k$ yields

$$
\sum_{k=1}^{l}( \pm 1)^{k} x^{a k+b-1}=x^{a+b-1} \frac{\left( \pm x^{a}\right)^{l+1}-1}{\left( \pm x^{a}\right)-1} .
$$


This representation is applied to the innermost sum $\left(a=a_{l}, b=b_{l}, c=c_{l}\right)$. One now may perform the next sum in the same way, provided $a_{l-1} \mid a_{l}$. If this is not the case, one transforms the integration variables in (2.4) and (2.5), such that the next denominator can be generated, etc. In this way, the sums (2.1) can be represented in terms of linear combinations of Poincaré-iterated integrals. Evidently, the representation of the cyclotomic harmonic sum (2.1) in terms of a (properly regularized) Mellin transform will be related to the Mellin variable $k N$, with $k$ being the least common multiple of $a_{1}, \ldots, a_{l}$.

Let us illustrate the principle steps in case of the following example:

$$
S_{\{3,2,2\},\{2,1,1\}}(1,-1 ; N)=\sum_{k=1}^{N} \frac{1}{(3 k+2)^{2}} \sum_{l=1}^{k} \frac{(-1)^{l}}{(2 l+1)} .
$$

The first sum yields

$$
S_{\{3,2,2\},\{2,1,1\}}(1,-1 ; N)=\sum_{k=1}^{N} \int_{0}^{1} d x \frac{x^{2}}{x^{2}+1} \frac{\left(-x^{2}\right)^{k}+1}{(3 k+2)^{2}} .
$$

Setting $x=y^{3}$, one obtains

$$
\begin{aligned}
S_{\{3,2,2\},\{2,1,1\}}(1,-1 ; N)= & 12 \int_{0}^{1} d y \frac{y^{8}}{y^{6}+1} \sum_{k=1}^{N} \frac{\left(-y^{6}\right)^{k}-1}{(6 k+4)^{2}} \\
= & 12 \int_{0}^{1} d y \frac{y^{4}}{y^{6}+1}\left\{\int_{0}^{y} \frac{d z}{z} \int_{0}^{z} d t t^{9} \frac{\left(-t^{6}\right)^{N}-1}{t^{6}+1}\right. \\
& \left.\quad-y^{4} \int_{0}^{1} \frac{d z}{z} \int_{0}^{z} d t t^{9} \frac{t^{6 N}-1}{t^{6}-1}\right\} \\
= & 12 \int_{0}^{1} d y \frac{y^{4}}{y^{6}+1} \int_{0}^{y} \frac{d z}{z} \int_{0}^{z} d t t^{9} \frac{\left(-t^{6}\right)^{N}-1}{t^{6}+1} \\
& \left.-(4-\pi) \int_{0}^{1} \frac{d z}{z} \int_{0}^{z} d t t^{9} \frac{t^{6 N}-1}{t^{6}-1}\right\} .
\end{aligned}
$$

In general, the polynomials

$$
x^{a}-1
$$

in (2.6) decompose in a product of cyclotomic polynomials, except for $a=1$ for which the expression is $\Phi_{1}(x)$, see Sec. III. Moreover, the polynomials

$$
x^{a}+1=\frac{x^{2 a}-1}{x^{a}-1}
$$

are either cyclotomic for $a=2^{n}, n \in \mathbb{N}$ or decompose into products of cyclotomic polynomials in other cases, see Appendix A. All factors divide $\left(x^{a}\right)^{l}-1$, respectively, $\left(-x^{a}\right)^{l}-1$. We remark that Eq. (2.10) is not yet written in terms of a Mellin transform (1.2). To achieve this in an automatic fashion, the cyclotomic harmonic polylogarithms are introduced in Sec. III. Furthermore, their special values at $x=1$ contribute to which we turn in Sec. V.

\section{CYCLOTOMIC HARMONIC POLYLOGARITHMS}

To account for the newly emerging sums (2.1) in perturbative calculations in quantum field theory, we introduce Poincaré-iterated integrals over the alphabet $\mathfrak{A}$,

$$
\mathfrak{A}=\left\{\frac{1}{x}\right\} \cup\left\{\frac{x^{l}}{\Phi_{k}(x)} \mid k \in \mathbb{N}_{+}, 0 \leq l<\varphi(k)\right\}
$$


where $\Phi_{k}(x)$ denotes the $k$ th cyclotomic polynomial, ${ }^{23}$ and $\varphi(k)$ denotes Euler's totient function: ${ }^{24}$

$$
\Phi_{n}(x)=\frac{x^{n}-1}{\prod_{d \mid n, d<n} \Phi_{d}(x)}, \quad d, n \in \mathbb{N}_{+},
$$

and the first cyclotomic polynomials are given by

$$
\begin{aligned}
& \Phi_{1}(x)=x-1, \\
& \Phi_{2}(x)=x+1 \\
& \Phi_{3}(x)=x^{2}+x+1, \\
& \Phi_{4}(x)=x^{2}+1 \\
& \Phi_{5}(x)=x^{4}+x^{3}+x^{2}+x+1, \\
& \Phi_{12}(x)=x^{4}-x^{2}+1, \text { etc. } \\
& \Phi_{11}(x)=x^{10}+x^{9}+x^{8}+x^{7}+x^{6}+x^{5}+x^{4}+x^{3}+x^{2}+x+1, \\
& \Phi_{7}(x)=x^{6}+x^{5}+x^{4}+x^{3}+x^{2}+x+1, \\
& \Phi^{6}(x)=x^{4}+1
\end{aligned}
$$

The alphabet $\mathfrak{A}$ is an extension of the alphabet

$$
\mathfrak{A}_{\mathrm{H}}=\left\{\frac{1}{x}, \frac{1}{\Phi_{1}(x)}, \frac{1}{\Phi_{2}(x)}\right\},
$$

generating the usual harmonic polylogarithms. ${ }^{26,88}$ As a shorthand notation we define the letters of $\mathfrak{A}$ by

$$
\begin{aligned}
& f_{0}^{0}(x)=\frac{1}{x}, \\
& f_{k}^{l}(x)=\frac{x^{l}}{\Phi_{k}(x)}, \quad k \in \mathbb{N}_{+}, l \in \mathbb{N}, l \leq \varphi(k) .
\end{aligned}
$$


Here, the labels $k$ and $l$ form a double index, which always appears at a common position. Mellin transformations associated to $1 / \Phi_{4}(x)$ and $1 / \Phi_{6}(x)$ were discussed long ago in Ref. 36,

$$
\begin{aligned}
\frac{1}{2} \beta\left(\frac{x}{2}\right) & =\int_{0}^{1} d t \frac{t^{x-1}}{t^{2}+1}, \\
\beta\left(\frac{x}{3}\right) & =\beta(x)-\int_{0}^{1} d t t^{x-1} \frac{t-2}{t^{2}-t+1}
\end{aligned}
$$

with Stirling's $\beta$-function, ${ }^{37}$

$$
\beta(x)=\frac{1}{2}\left[\Psi\left(\frac{x+1}{2}\right)-\Psi\left(\frac{x}{2}\right)\right] .
$$

Integrals of this kind emerge also in particle physics problems in Refs. 20 and 38-40.

We form the Poincaré iterated integrals,

$$
\begin{aligned}
C_{k_{1}, \ldots, k_{m}}^{l_{1}, \ldots, l_{m}}(z) & =\frac{1}{m !} \ln (x)^{m}, & & \text { if }\left(l_{1}, \ldots, l_{m}\right)=(0, \ldots, 0),\left(k_{1}, \ldots, k_{m}\right)=(0, \ldots, 0), \\
C_{k_{m}}^{l_{m}}(z) & =\int_{0}^{z} d x f_{k_{m}}^{l_{m}}(x), & & \text { if } k_{m} \neq 0, \\
C_{k_{1}, \ldots, k_{m}}^{l_{1}, \ldots, l_{m}}(z) & =\int_{0}^{z} d x f_{k_{1}}^{l_{1}}(x) C_{k_{2}, \ldots, k_{m}}^{l_{2}, \ldots, l_{m}}(x), & & \text { if }\left(k_{1}, \ldots, k_{m}\right) \neq(0, \ldots, 0),
\end{aligned}
$$

and $C_{\vec{a}} \vec{l}(z)$ denotes cyclotomic harmonic polylogarithms of weight $\mathrm{w}=\mathrm{m}$. They form a shuffle algebra ${ }^{9,41}$ by multiplication

$$
\begin{aligned}
C_{\vec{a}_{1}}^{\vec{a}_{2}}(z) \cdot C_{\vec{b}_{1}}^{\vec{b}_{2}}(z)=C_{\vec{a}_{1}}^{\vec{a}_{2}}(z) \amalg C_{\vec{b}_{1}}^{\vec{b}_{2}}(z)= & \sum_{\left[\begin{array}{l}
\vec{c}_{2} \\
\vec{c}_{1}
\end{array}\right] \in\left[\begin{array}{l}
\vec{a}_{2} \\
\vec{a}_{1}
\end{array}\right] \uplus\left[\begin{array}{l}
\vec{b}_{2} \\
\vec{b}_{1}
\end{array}\right]} C_{\vec{c}_{1}}^{\vec{c}_{2}}(z)
\end{aligned}
$$

of $M^{\mathrm{w}}$ elements at weight $\mathrm{w}$, where $M$ denotes the number of chosen letters from $\mathfrak{A}$. The shuffle symbol $\amalg$ implies all combinations of indices $a_{i j} \in \vec{a}_{i}$ and $b_{i j} \in \vec{b}_{j}$ leaving the order in both sets unchanged and the brackets [] pair the upper and lower indices forming a unity, cf. (3.17).

The number of basis elements spanning the shuffle algebra is given by

$$
N^{\text {basic }}(\mathbf{w})=\frac{1}{\mathrm{w}} \sum_{d \mid \mathbf{w}} \mu\left(\frac{\mathrm{w}}{d}\right) M^{d}, \quad \mathrm{w} \geq 1,
$$

according to the 1 st Witt formula. ${ }^{41,42}$ Here, $\mu$ denotes the Möbius function. ${ }^{43}$ The number of basic cyclotomic harmonic polylogarithms in dependence of $\mathrm{W}$ and $M$ is given in Table $\mathrm{I}$.

Now, the cyclotomic harmonic sums (2.1) can be represented as Mellin transforms of cyclotomic harmonic polylogarithms. In the example (2.10) the iterated integrals have to be rewritten. We express

TABLE I. Number of basic cyclotomic harmonic polylogarithms in dependence of the number of letters and weight.

\begin{tabular}{lrrrrrrr}
\hline \hline \multirow{2}{*}{ Weight } & \multicolumn{7}{c}{ Letters } \\
\cline { 2 - 8 } & 2 & 3 & 4 & 5 & 6 & 7 & 8 \\
\hline 1 & 2 & 3 & 4 & 5 & 6 & 7 & 8 \\
2 & 1 & 3 & 6 & 10 & 15 & 21 & 168 \\
3 & 2 & 8 & 20 & 40 & 70 & 112 & 1008 \\
4 & 3 & 18 & 60 & 150 & 315 & 588 & 6552 \\
5 & 6 & 48 & 204 & 624 & 1554 & 3360 & 43596 \\
6 & 9 & 116 & 670 & 2580 & 7735 & 19544 & 299592 \\
7 & 18 & 312 & 2340 & 11160 & 39990 & 117648 & 2096640 \\
8 & 30 & 810 & 8160 & 48750 & 209790 & 729300 & 2006 \\
\hline \hline
\end{tabular}


respective denominators in terms of products of cyclotomic polynomials, (A2)-(A16), and perform partial fractioning in the respective integration variable, (A27)-(A38). In our concrete example, the integrand of the $y$-integral in (2.9) has the representation

$$
\frac{y^{4}}{y^{6}+1}=\frac{1}{3}\left[f_{4}^{0}(y)-f_{12}^{0}(y)+2 f_{12}^{2}(y)\right] \text {. }
$$

With integration by parts, one obtains the following Mellin transforms of argument $6 \mathrm{~N}$ of cyclotomic harmonic polylogarithms $C_{k_{1}, \ldots, k_{m}}^{l_{1}, \ldots, l_{m}}(x)$ weighted by the letters $f_{l}^{k}(x)$ of the alphabet $\mathfrak{A}$ :

$$
\begin{aligned}
& S_{\{3,2,2\},\{2,1,1\}}(1,-1 ; N)= \frac{1}{6}(4-\pi) \int_{0}^{1} d x x^{3}\left(x^{6 N}-1\right)\left[6+f_{1}^{0}(x)-f_{2}^{0}(x)-2 f_{3}^{0}(x)\right. \\
&\left.-f_{3}^{1}(x)-2 f_{6}^{0}(x)+f_{6}^{1}(x)\right] C_{0}^{0}(x) \\
&-2 \int_{0}^{1} d x x^{3}\left[(-1)^{N} x^{6 N}-1\right]\left[3-f_{4}^{0}(x)-2 f_{12}^{0}(x)+2 f_{12}^{2}(x)\right] C_{0}^{0}(x) \\
&-\frac{4}{3}\left[C_{0,4}^{0,0}(1)-C_{0,12}^{0,0}(1)+2 C_{0,12}^{0,2}(1)\right] \int_{0}^{1} d x x^{3}\left[(-1)^{N} x^{6 N}-1\right] \\
& \times\left[3-f_{4}^{0}(x)-2 f_{12}^{0}(x)+2 f_{12}^{2}(x)\right] \\
&+ \frac{4}{3} \int_{0}^{1} d x x^{3}\left[(-1)^{N} x^{6 N}-1\right]\left[C_{0,4}^{0,0}(x)-C_{0,12}^{0,0}(x)+2 C_{0,12}^{0,2}(x)\right] \\
& \times\left[3-f_{4}^{0}(x)-2 f_{12}^{0}(x)+2 f_{12}^{2}(x)\right] .
\end{aligned}
$$

The constants $C_{k_{1}, \ldots, k_{m}}^{l_{1}, \ldots, l_{m}}(1)$ are discussed in Sec. V. In particular, one obtains

$$
C_{0,4}^{0,0}=-\mathbf{C}
$$

with $\mathbf{C}$ being the Catalan number, ${ }^{44}$ and $C_{0,12}^{0,0}$ and $C_{0,12}^{0,2}$ being linear combinations of $\psi^{\prime}(1 / 12)$ and $\psi^{\prime}(5 / 12)$. The latter numbers reduce further, cf. Sec. V. Note that in all cases in which neither $f_{1}^{0}(z)$ nor $C_{1, k_{2}, \ldots, k_{m}}^{0, l_{2} \ldots, l_{m}}(z)$ are present, the $z$-independent terms in $\left[\left( \pm z^{k}\right)^{N}-1\right]$ can be integrated, since the other cyclotomic letters $f_{l}^{k}(z)$ and $C_{k, k_{2}, \ldots, k_{m}}^{l, l_{2} \ldots, l_{m}}(z)$ for $k>1$ are regular at $z=1$.

In general, linear combinations of the Mellin transforms

$$
\mathbf{M}\left[f_{c}^{d}(x) \cdot C_{\vec{a}}^{\vec{b}}(x)\right](l N)=\int_{0}^{1} d x x^{l N}\left(f_{c}^{d}(x)\right)^{u} \cdot C_{\vec{a}}^{\vec{b}}(x), \quad u \in\{0,1\}
$$

with $l$ being the least common multiple of $a_{1}, \ldots, a_{k}$ allow to represent all cyclotomic harmonic sums. Summarizing, we can write

$$
S_{\left\{a_{1}, b_{1}, c_{1}\right\}, \ldots,\left\{a_{k}, b_{k}, c_{k}\right\}}(N)=\sum_{n=1}^{s} e_{n} \int_{0}^{1} d x x^{l N}\left(f_{\alpha_{n}}^{\beta_{n}}(x)\right)^{u_{n}} C_{\vec{\gamma}_{n}}^{\overrightarrow{\delta_{n}}}(x)
$$

with $e_{n} \in \mathbb{R}$ and $u_{n} \in\{0,1\}$; here, $e_{n}$ is determined by polynomial expressions in terms of cyclotomic harmonic polylogarithms evaluated at 1 with rational coefficients. Within this transformation and in the following the letter $1 / x$ plays a special role concerning the cyclotomic harmonic sums. We can exclude the case that the first letter in (3.26) is $1 / x$, since it would just shift the Mellin index by one unit. In case of $\Phi_{1}(x)$ and related functions the +-regularization

$$
\mathbf{M}\left[\left(\frac{f(x)}{x-1}\right)_{+}\right](N)=\int_{0}^{1} d x \frac{x^{N}-1}{x-1} f(x)
$$

is applied to (3.26). Only in case that $f_{1}(x)=1 /(x-1)$ and $C_{\vec{a}}(x)$ do not vanish in the limit $x \rightarrow 1$, a + -function must occur. Iterations of cyclotomic letters $f_{k}^{l}(x), k \geq 1,0 \leq l<k$ for $k \geq 2$ have no singularities in $x \in[0,1]$. 
We remark that the sketched transformation from cyclotomic harmonic sums to their Mellin transforms in terms of cyclotomic harmonic polylogarithms can be reversed, i.e., a given expression in terms of Mellin transforms of cyclotomic harmonic polylogarithms can be expressed in terms of cyclotomic harmonic sums. The explicit algorithms have been worked out in details for the alphabet

$$
\mathfrak{A}^{\prime}:=\left\{f_{0}^{0}, f_{1}^{0}, f_{2}^{0}, f_{4}^{0}, f_{4}^{1}\right\}=\left\{\frac{1}{x}, \frac{1}{\Phi_{1}(x)}, \frac{1}{\Phi_{2}(x)}, \frac{1}{\Phi_{4}(x)}, \frac{x}{\Phi_{4}(x)}\right\} \subseteq \mathfrak{A},
$$

which allows one to express the cyclotomic harmonic sums $S_{\left\{a_{1}, b_{1}, c_{1}\right\}, \ldots,\left\{a_{l}, b_{l}, c_{l}\right\}}\left(s_{1}, \ldots, s_{l} ; N\right)$ with $a_{i} \in\{1,2\}, b_{i} \in\{0,1\}$, and $c_{i} \in \mathbb{N}_{+}$in terms of Mellin transforms of cyclotomic harmonic polylogarithms in both directions; the implementation is available within the HARMONICSUMS package. ${ }^{27}$ This transformation will be used, e.g., in Sec. IV A.

The Mellin transforms (3.26) and (3.28) obey difference equations of order $l$ in $N$, which can be used to define these functions specifying respective initial values for $l$ moments. In the following we illustrate this for the words $x^{l} / \Phi_{k}(x)$. One obtains

$$
\sum_{n=0}^{N_{k}} c_{n, k} \phi_{k}(N+n-l)=\frac{1}{N+1},
$$

where $\Phi_{k}(x)$ is given by

$$
\Phi_{k}(x)=\sum_{n=0}^{N_{k}} c_{n, k} x^{n} .
$$

Here, we define

$$
\begin{gathered}
\phi_{1}(0, N)=\int_{0}^{1} d x \frac{x^{N}-1}{x-1}, \\
\phi_{k}(l, N)=\int_{0}^{1} d x x^{N} f_{k}^{l}(x), \quad \phi_{k}(N)=\phi_{k}(0, N), \quad k \geq 2, \\
\phi_{k}(l, N)_{+}=\int_{0}^{1} d x\left[x^{N}-1\right] f_{k}^{l}(x), \quad \phi_{k}(N)_{+}=\phi_{k}(0, N)_{+} .
\end{gathered}
$$

For $k<105$ for all coefficients, $c_{n, k} \in\{-1,0,1\}$ holds. ${ }^{45}$ One derives the following first order difference equations (3.30) for Mellin transforms associated to the lowest order cyclotomic polynomials:

$$
\begin{aligned}
\phi_{1}(l, N+1)-\phi_{1}(l, N) & =\frac{1}{N+l+1}, \\
\phi_{2}(l, N+1)+\phi_{2}(l, N) & =\frac{1}{N+l+1}, \\
\phi_{3}(l, N+3)-\phi_{3}(l, N) & =-\frac{1}{(N+l+1)(N+l+2)}, \\
\phi_{4}(l, N+2)+\phi_{4}(l, N) & =\frac{1}{N+l+1}, \\
\phi_{5}(l, N+5)-\phi_{5}(l, N) & =-\frac{1}{(N+l+1)(N+l+2)}, \\
\phi_{6}(l, N+3)+\phi_{6}(l, N) & =\frac{2(N+l)+3}{(N+l+1)(N+l+2)},
\end{aligned}
$$




$$
\begin{aligned}
\phi_{7}(l, N+7)-\phi_{7}(l, N) & =-\frac{1}{(N+l+1)(N+l+2)}, \\
\phi_{8}(l, N+4)-\phi_{8}(l, N) & =\frac{1}{N+l+1}, \\
\phi_{9}(l, N+9)-\phi_{9}(l, N) & =-\frac{3}{(N+l+1)(N+l+4)}, \\
\phi_{10}(l, N+5)+\phi_{10}(l, N) & =\frac{3(N+l)+2}{(N+l+1)(N+l+2)}, \\
\phi_{11}(l, N+11)-\phi_{11}(l, N) & =\frac{1}{(N+l+1)(N+l+2)}, \\
\phi_{12}(l, N+6)+\phi_{12}(l, N) & =\frac{3(N+l)+2}{(N+l+1)(N+l+2)}, \quad \text { etc. }
\end{aligned}
$$

Together with the corresponding initial values, these recurrence relations enable one to compute efficiently the values for $N$. In particular, due to the special form of the recurrences, we get explicit representations in terms of finite sums. For example, for $\phi_{6}(l, N)$, we obtain

$$
\phi_{6}(l, 3 N)=(-1)^{N}\left(\sum_{i=1}^{N} \frac{(-1)^{i}(2(3 i+l)-3)}{(3(i+l)-2)(3(i+l)-1)}+\phi_{6}(l, 0)\right),
$$

and

$$
\begin{aligned}
& \phi_{6}(l, 3 N+1)=\phi_{6}(l+1,3 N), \\
& \phi_{6}(l, 3 N+2)=\phi_{6}(l+2,3 N) .
\end{aligned}
$$

Looking at

$$
\lim _{N \rightarrow \infty} \phi_{6}(l, N)=\lim _{N \rightarrow \infty} \int_{0}^{1} x^{N+l} \frac{1}{x^{2}-x-1} d x=\lim _{N \rightarrow \infty} \int_{0}^{1} x^{N+l}\left(1+x-x^{3}-x^{4}+\ldots\right) d x=0
$$

shows that

$$
\phi_{6}(l, 0)=-\sum_{i=1}^{\infty} \frac{(-1)^{i}[2(3 i+l)-3]}{(3 i+l-2)(3 i+l-1)} .
$$

Completely analogously, all the other functions (3.35) to (3.46) can be written in such a sum representation where the constants are the infinite versions of it multiplied with a minus sign. Note that these constants can be written as a linear combination of the infinite sums

$$
\sigma_{\{a, b, s\}}=\sum_{k=1}^{\infty} \frac{s^{k}}{a k+b}
$$

with $s \in\{-1,1\}$ and $a, b \in \mathbb{N}$ with $a \neq 0$. The relevant values for this paper will be worked out explicitly in Sec. V.

The $\phi_{k}(l, N)_{+}$functions are preferred if the recurrences (3.35) to (3.46) form telescoping equations. In this case, in particular, if $k$ is odd, the $\phi_{k}(l, r N)$ for properly chosen $r$ can be related to sums without any extra constant, e.g.,

$$
\phi_{5}(l, 5 N)_{+}=-\sum_{i=1}^{N} \frac{1}{(5 i+l-4)(5 i+l-3)} .
$$


$\phi_{1}$ and $\phi_{2}$ can be related to the single cyclotomic harmonic sums at weight $\mathrm{w}=1$ as follows:

$$
\begin{aligned}
\phi_{1}(l, N)_{+} & =S_{1}(N+l), \\
\phi_{2}(l, N) & =(-1)^{N}\left[S_{-1}(N+l)-\ln (2)+S_{-1}(l)\right] .
\end{aligned}
$$

For later considerations we use $S_{1}(N)$ and $S_{-1}(N)$ instead of $\phi_{1}(N)$ and $\phi_{2}(N)$. In Sec. IV the single cyclotomic harmonic sums of weight $\mathbf{W}=1$ are expressed in terms of the Mellin transforms $\phi_{k}(l, r N)$ for properly chosen $r$. In addition, the cyclotomic harmonic sums of higher weight and depth will be discussed.

\section{CYCLOTOMIC HARMONIC SUMS}

We consider the extension of the finite nested harmonic sums ${ }^{7,8}$ to those generated by the cyclotomic harmonic polylogarithms discussed in Sec. III. First, the single cyclotomic harmonic sums are considered and explicit representations are given. We derive their analytic continuation to complex values of $N$. Next, the algebraic, differential, and three multiple argument relations of the cyclotomic harmonic sums are discussed. These relations are used to represent these sums over suitable bases. Finally, we consider the nested sums over the alphabet $\left\{( \pm 1)^{k} / k,( \pm 1)^{k} /(2 k+1)\right\}$ to higher weight deriving explicit relations up to $w=5$.

\section{A. The single sums}

The single cyclotomic harmonic sums are given by

$$
\sum_{k=0}^{N} \frac{( \pm 1)^{k}}{(l \cdot k+m)^{n}} .
$$

Here, $N$ is either an even or an odd integer. In case one needs representations only for $N \in \mathbb{N}$, the following representations hold:

$$
\begin{aligned}
& \sum_{k=0}^{\bar{N}} \frac{(-1)^{k}}{l \cdot k+m}=\left[\sum_{k=0}^{N} \frac{1}{(2 l) \cdot k+m}-\frac{1}{(2 l) \cdot k+m+l}\right] \\
& \sum_{k=0}^{\bar{N}+1} \frac{(-1)^{k}}{l \cdot k+m}=\left[\sum_{k=0}^{N} \frac{1}{(2 l) \cdot k+m}-\frac{1}{(2 l) \cdot k+m+l}\right]-\frac{1}{(2 l) N+l+m}
\end{aligned}
$$

with $\bar{N}=2 N$. However, one is interested in relations for general values of $N$, since for nested sums more and more cases have to be distinguished. The single sums can be expressed in terms of the Mellin transforms $\phi_{k}(l, k N),(3.32)$ and (3.33). Up to $\mathrm{w}=6$, one obtains

$$
\begin{aligned}
& \sum_{k=1}^{N} \frac{1}{1+2 k}=-\frac{2 N}{2 N+1}-\frac{S_{1}(N)}{2}+S_{1}(2 N), \\
& \sum_{k=1}^{N} \frac{(-1)^{k}}{1+2 k}=(-1)^{N}\left[\frac{1}{2 N+1}-\phi_{4}(2 N)\right]+\sigma_{\{2,1,-1\}}, \\
& \sum_{k=1}^{N} \frac{1}{1+3 k}=-\frac{3 N}{3 N+1}-\frac{S_{1}(N)}{6}+\frac{1}{2} S_{1}(3 N)-\frac{1}{2} \phi_{3}(3 N)_{+},
\end{aligned}
$$




$$
\begin{aligned}
& \sum_{k=1}^{N} \frac{(-1)^{k}}{1+3 k}=\frac{1}{6} S_{-1}(N)-\frac{1}{2} S_{-1}(3 N)+(-1)^{N}\left[\frac{1}{3 N+1}-\frac{1}{2} \phi_{6}(3 N)\right]+\frac{1}{3} \sigma_{\{1,0,-1\}}+\sigma_{\{3,1,-1\}}, \\
& \sum_{k=1}^{N} \frac{1}{2+3 k}=-\frac{3 N}{2(3 N+2)}-\frac{S_{1}(N)}{6}+\frac{1}{2} S_{1}(3 N)+\frac{1}{2} \phi_{3}(3 N)_{+}, \\
& \sum_{k=1}^{N} \frac{(-1)^{k}}{2+3 k}=-\frac{1}{6} S_{-1}(N)+\frac{1}{2} S_{-1}(3 N)+(-1)^{N}\left[\frac{1}{3 N+2}-\frac{1}{2} \phi_{6}(3 N)\right] \\
& +\frac{1}{3} \sigma_{\{1,0,-1\}}+\sigma_{\{3,1,-1\}}+\frac{1}{2}, \\
& \sum_{k=1}^{N} \frac{1}{1+4 k}=-\frac{2 N}{4 N+1}-\frac{1}{4} S_{1}(2 N)+\frac{1}{2} S_{1}(4 N)-\frac{1}{2} \phi_{4}(4 N)+\frac{1}{2} \sigma_{\{2,1,-1\}}+\frac{1}{2(4 N+1)}, \\
& \sum_{k=1}^{N} \frac{(-1)^{k}}{1+4 k}=(-1)^{N}\left[\frac{1}{4 N+1}-\phi_{8}(4 N)\right]+\sigma_{\{4,1,-1\}} \\
& \sum_{k=1}^{N} \frac{1}{3+4 k}=-\frac{10 N}{3(4 N+3)}-\frac{1}{4} S_{1}(2 N)+\frac{1}{2} S_{1}(4 N)+\frac{1}{2} \phi_{4}(4 N)-\frac{1}{2} \sigma_{\{2,1,-1\}}-\frac{3}{2(4 N+3)}, \\
& \sum_{k=1}^{N} \frac{(-1)^{k}}{3+4 k}=(-1)^{N}\left[\frac{1}{4 N+3}-\phi_{8}(2,4 N)\right]+\sigma_{\{4,3,-1\}}, \\
& \sum_{k=1}^{N} \frac{1}{1+5 k}=-\frac{5 N}{5 N+1}-\frac{S_{1}(N)}{20}+\frac{1}{4} S_{1}(5 N)-\frac{3}{4} \phi_{5}(5 N)_{+}-\frac{1}{2} \phi_{5}(1,5 N)_{+}-\frac{1}{4} \phi_{5}(2,5 N)_{+}, \\
& \sum_{k=1}^{N} \frac{(-1)^{k}}{1+5 k}=(-1)^{N}\left[-\frac{3}{4} \phi_{10}(5 N)+\frac{1}{2} \phi_{10}(1,5 N)-\frac{1}{4} \phi_{10}(2,5 N)+\frac{1}{5 N+1}\right] \\
& +\frac{1}{20} S_{-1}(N)-\frac{1}{4} S_{-1}(5 N)+\frac{1}{5} \sigma_{\{1,0,-1\}}+\sigma_{\{5,1,-1\}}, \\
& \sum_{k=1}^{N} \frac{1}{2+5 k}=-\frac{5 N}{2(5 N+2)}-\frac{S_{1}(N)}{20}+\frac{1}{4} S_{1}(5 N)+\frac{1}{4} \phi_{5}(5 N)_{+}-\frac{1}{2} \phi_{5}(1,5 N)_{+}-\frac{1}{4} \phi_{5}(2,5 N)_{+}, \\
& \sum_{k=1}^{N} \frac{(-1)^{k}}{2+5 k}=(-1)^{N}\left[-\frac{1}{4} \phi_{10}(5 N)-\frac{1}{2} \phi_{10}(1,5 N)+\frac{1}{4} \phi_{10}(2,5 N)+\frac{1}{5 N+2}\right] \\
& -\frac{1}{20} S_{-1}(N)+\frac{1}{4} S_{-1}(5 N)-\frac{1}{5} \sigma_{\{1,0,-1\}}+\sigma_{\{5,2,-1\}}, \\
& \sum_{k=1}^{N} \frac{1}{3+5 k}=-\frac{5 N}{3(5 N+3)}-\frac{S_{1}(N)}{20}+\frac{1}{4} S_{1}(5 N)+\frac{1}{4} \phi_{5}(5 N)_{+}+\frac{1}{2} \phi_{5}(1,5 N)_{+}-\frac{1}{4} \phi_{5}(2,5 N)_{+},
\end{aligned}
$$




$$
\begin{aligned}
\sum_{k=1}^{N} \frac{(-1)^{k}}{3+5 k}= & (-1)^{N}\left[\frac{1}{4} \phi_{10}(5 N)-\frac{1}{2} \phi_{10}(1,5 N)-\frac{1}{4} \phi_{10}(2,5 N)+\frac{1}{5 N+3}\right] \\
& +\frac{1}{20} S_{-1}(N)-\frac{1}{4} S_{-1}(5 N)+\frac{1}{5} \sigma_{\{1,0,-1\}}+\sigma_{\{5,3,-1\}},
\end{aligned}
$$

$$
\begin{aligned}
\sum_{k=1}^{N} \frac{1}{4+5 k}= & -\frac{5 N}{4(5 N+4)}-\frac{S_{1}(N)}{20}+\frac{1}{4} S_{1}(5 N)+\frac{1}{4} \phi_{5}(5 N)_{+} \\
& +\frac{1}{2} \phi_{5}(1,5 N)_{+}+\frac{3}{4} \phi_{5}(2,5 N)_{+},
\end{aligned}
$$

$$
\begin{aligned}
\sum_{k=1}^{N} \frac{(-1)^{k}}{4+5 k}= & (-1)^{N}\left[-\frac{1}{4} \phi_{10}(5 N)+\frac{1}{2} \phi_{10}(1,5 N)-\frac{3}{4} \phi_{10}(2,5 N)+\frac{1}{5 N+4}\right] \\
& -\frac{1}{20} S_{-1}(N)+\frac{1}{4} S_{-1}(5 N)+\frac{3}{5} \sigma_{\{1,0,-1\}}+\sigma_{\{5,1,-1\}}-\sigma_{\{5,2,-1\}}+\sigma_{\{5,3,-1\}}+\frac{7}{12}
\end{aligned}
$$

$$
\sum_{k=1}^{N} \frac{1}{1+6 k}=-\frac{6 N}{6 N+1}+\frac{S_{1}(N)}{12}-\frac{1}{6} S_{1}(2 N)-\frac{1}{4} S_{1}(3 N)+\frac{1}{2} S_{1}(6 N)-\frac{1}{4} \phi_{3}(3 N)_{+}-\frac{1}{2} \phi_{3}(6 N)_{+},
$$

$$
\begin{aligned}
\sum_{k=1}^{N} \frac{(-1)^{k}}{1+6 k}= & (-1)^{N}\left[-\phi_{12}(6 N)+\frac{1}{3} \phi_{4}(2 N)+\frac{1}{6 N+1}\right]+\sigma_{\{6,1,-1\}}, \\
\sum_{k=1}^{N} \frac{1}{5+6 k}= & -\frac{6 N}{5(6 N+5)}+\frac{S_{1}(N)}{12}-\frac{1}{6} S_{1}(2 N)-\frac{1}{4} S_{1}(3 N)+\frac{1}{2} S_{1}(6 N) \\
& +\frac{1}{4} \phi_{3}(3 N)_{+}+\frac{1}{2} \phi_{3}(6 N)_{+} \\
\sum_{k=1}^{N} \frac{(-1)^{k}}{5+6 k}= & (-1)^{N}\left[\phi_{12}(6 N)-\frac{2}{3} \phi_{4}(2 N)-\phi_{4}(6 N)+\frac{1}{6 N+5}\right]+\frac{4}{3} \sigma_{\{2,1,-1\}}-\sigma_{\{6,1,-1\}}+\frac{2}{15}
\end{aligned}
$$

Taking the sum representations, such as (3.50) and (3.47) given by the recurrence relations (3.35) to (3.46) and the corresponding initial values $\phi_{k}(l, 0)$ (which are expressible by the infinite sums $\sigma_{\{a, b, \pm 1\}}$, cf. Sec. V), we used the summation package SIGMA (Ref. 17) to perform this transformation. As a consequence, the single cyclotomic harmonic sums can be expressed in terms of the sums

$$
\begin{aligned}
& S_{-1}(N), S_{-1}(3 N), S_{-1}(5 N), S_{1}(N), S_{1}(2 N), S_{1}(3 N), S_{1}(4 N), S_{1}(5 N), S_{1}(6 N), \\
& \phi_{3}(3 N)_{+}, \phi_{3}(6 N)_{+}, \phi_{4}(2 N), \phi_{4}(4 N), \phi_{4}(6 N), \phi_{5}(5 N)_{+}, \phi_{5}(1,5 N)_{+}, \\
& \phi_{5}(2,5 N)_{+}, \phi_{6}(3 N), \phi_{8}(4 N), \phi_{8}(2,4 N), \phi_{10}(5 N), \phi_{10}(1,5 N), \phi_{10}(2,5 N), \phi_{12}(6 N) .
\end{aligned}
$$

In particular, in the way how this construction is carried out it follows by the summation theory of Ref. 17 that the sequences produced by these sums form an algebraic independent basis over the ring of sequences generated by the elements from $\mathbb{R}(N)\left[(-1)^{N}\right]$. Note that we exploited the algebraic relations (5.118) to (5.129) which implies that only the following constants

$$
\sigma_{\{1,0,-1\}}, \sigma_{\{2,1,-1\}}, \sigma_{\{3,1,-1\}}, \sigma_{\{4,1,-1\}}, \sigma_{\{4,3,-1\}}, \sigma_{\{5,1,-1\}}, \sigma_{\{5,2,-1\}}, \sigma_{\{5,3,-1\}} \sigma_{\{6,1,-1\}}
$$


from $\mathbb{R}$ appear in the representation found for the single cyclotomic harmonic sums with weight $\mathrm{w}=1$.

Due to (3.33) and (3.34), the functions $\Phi_{k}(l, N), k \geq 1$ can be represented as factorial series. ${ }^{36,46}$ Therefore, they are meromorphic functions in $N$ with poles at $-n, n \in \mathbb{N}$. This also applies to $\phi_{k}(0, N)$, (3.32). The latter function grows $\propto \ln (N)$ for $N \rightarrow \infty,|\arg (N)|<\pi$. The recursion relations (3.30) allow one to shift $\phi_{k}(l, N)$ in $N \rightarrow N+1$. To represent a function $\phi_{k}(l, N)$ for $N \in \mathbb{C}$, one needs to know its asymptotic representation in addition.

It is given in analytic form in terms of series involving the Stirling numbers of the 2nd kind. ${ }^{36,37}$ The corresponding representations read

$$
\begin{aligned}
& \phi_{1}(0, N) \sim \gamma+\ln (N)+\frac{1}{2 N}-\frac{1}{12 N^{2}}+\frac{1}{120 N^{4}}-\frac{1}{252 N^{6}}+\frac{1}{240 N^{8}} \\
& -\frac{1}{132 N^{10}}+\frac{691}{32760 N^{12}}+O\left(\frac{1}{N^{13}}\right) \\
& \phi_{2}(0, N) \sim \frac{1}{2 N}-\frac{1}{4 N^{2}}+\frac{1}{8 N^{4}}-\frac{1}{4 N^{6}}+\frac{17}{16 N^{8}}-\frac{31}{4 N^{10}}+\frac{691}{8 N^{12}}+O\left(\frac{1}{N^{13}}\right), \\
& \phi_{3}(0, N) \sim \frac{1}{3 N}-\frac{2}{9 N^{3}}+\frac{2}{3 N^{5}}-\frac{14}{3 N^{7}}+\frac{1618}{27 N^{9}}-\frac{3694}{3 N^{11}}+O\left(\frac{1}{N^{13}}\right), \\
& \phi_{4}(0, N) \sim \frac{1}{2 N}-\frac{1}{2 N^{3}}+\frac{5}{2 N^{5}}-\frac{61}{2 N^{7}}+\frac{1385}{2 N^{9}}-\frac{50521}{2 N^{11}}+O\left(\frac{1}{N^{13}}\right), \\
& \phi_{5}(0, N) \sim \frac{1}{5 N}+\frac{1}{5 N^{2}}-\frac{1}{5 N^{3}}-\frac{1}{N^{4}}+\frac{31}{25 N^{5}}+\frac{67}{5 N^{6}}-\frac{109}{5 N^{7}}-\frac{361}{N^{8}}+\frac{3779}{5 N^{9}} \\
& +\frac{412751}{25 N^{10}}-\frac{214093}{5 N^{11}}-\frac{1150921}{N^{12}}+O\left(\frac{1}{N^{13}}\right) \text {, } \\
& \phi_{6}(0, N) \sim \frac{1}{N}-\frac{2}{N^{3}}+\frac{22}{N^{5}}-\frac{602}{N^{7}}+\frac{30742}{N^{9}}-\frac{2523002}{N^{11}}+O\left(\frac{1}{N^{13}}\right), \\
& \phi_{7}(0, N) \sim \frac{1}{7 N}+\frac{2}{7 N^{2}}-\frac{16}{7 N^{4}}-\frac{12}{7 N^{5}}+\frac{56}{N^{6}}+\frac{3900}{49 N^{7}}-\frac{20296}{7 N^{8}}-\frac{5796}{N^{9}} \\
& +\frac{1809992}{7 N^{10}}+\frac{4582500}{7 N^{11}}-\frac{35282968}{N^{12}}+O\left(\frac{1}{N^{13}}\right), \\
& \phi_{8}(0, N) \sim \frac{1}{2 N}+\frac{1}{2 N^{2}}-\frac{3}{2 N^{3}}-\frac{11}{2 N^{4}}+\frac{57}{2 N^{5}}+\frac{361}{2 N^{6}}-\frac{2763}{2 N^{7}}-\frac{24611}{2 N^{8}}+\frac{250737}{2 N^{9}} \\
& +\frac{2873041}{2 N^{10}}-\frac{36581523}{2 N^{11}}-\frac{512343611}{2 N^{12}}+O\left(\frac{1}{N^{13}}\right) \\
& \phi_{9}(0, N) \sim \frac{1}{3 N}+\frac{2}{3 N^{2}}-\frac{2}{3 N^{3}}-\frac{28}{3 N^{4}}+\frac{34}{3 N^{5}}+\frac{1172}{3 N^{6}}-\frac{1862}{3 N^{7}}-\frac{101428}{3 N^{8}}+\frac{207394}{3 N^{9}} \\
& +\frac{14999012}{3 N^{10}}-\frac{37996022}{3 N^{11}}-\frac{3386034628}{3 N^{12}}+O\left(\frac{1}{N^{13}}\right) \text {, }
\end{aligned}
$$




$$
\begin{aligned}
\phi_{10}(0, N) \sim & \frac{1}{N}+\frac{1}{N^{2}}-\frac{5}{N^{3}}-\frac{17}{N^{4}}+\frac{151}{N^{5}}+\frac{871}{N^{6}}-\frac{11465}{N^{7}}-\frac{92777}{N^{8}}+\frac{1626151}{N^{9}} \\
& +\frac{16922791}{N^{10}}-\frac{370714025}{N^{11}}-\frac{4715323337}{N^{12}}+O\left(\frac{1}{N^{13}}\right) \\
\phi_{11}(0, N) \sim & \frac{1}{11 N}+\frac{4}{11 N^{2}}+\frac{6}{11 N^{3}}-\frac{56}{11 N^{4}}-\frac{282}{11 N^{5}}+\frac{3064}{11 N^{6}}+\frac{26646}{11 N^{7}}-\frac{382616}{11 N^{8}} \\
& -\frac{4592442}{11 N^{9}}+\frac{7618184}{N^{10}}+\frac{13945859346}{121 N^{11}}-\frac{28200213176}{11 N^{12}}+O\left(\frac{1}{N^{13}}\right) \\
& +\frac{67637281}{N^{10}}-\frac{2237423527}{N^{11}}-\frac{27138236663}{N^{12}}+O\left(\frac{1}{N^{13}}\right) \\
\phi_{12}(0, N) \sim & \frac{1}{N}+\frac{1}{N^{2}}-\frac{7}{N^{3}}-\frac{23}{N^{4}}+\frac{305}{N^{5}}+\frac{1681}{N^{6}}-\frac{33367}{N^{7}}-\frac{257543}{N^{8}}+\frac{6815585}{N^{9}} \\
\phi_{5}(2, N) \sim & \frac{1}{5 N}-\frac{2}{5 N^{3}}+\frac{86}{25 N^{5}}-\frac{338}{5 N^{7}}+\frac{12094}{5 N^{9}}-\frac{690866}{5 N^{11}}+O\left(\frac{1}{N^{13}}\right) \\
\phi_{10}(2, N) \sim & \frac{1}{N}-\frac{6}{N^{3}}+\frac{186}{N^{5}}-\frac{14166}{N^{7}}+\frac{2009946}{N^{9}}-\frac{458225526}{N^{11}}+O\left(\frac{1}{N^{13}}\right) \\
\phi_{10}(1, N) \sim & \frac{1}{2 N}-\frac{2}{N^{3}}+\frac{40}{N^{5}}-\frac{1952}{N^{7}}+\frac{177280}{N^{9}}-\frac{25866752}{N^{11}}+O\left(\frac{1}{N^{13}}\right) \\
& \frac{16922791}{N^{3}}-\frac{17}{N^{4}}+\frac{151}{N^{5}}+\frac{871}{N^{6}}-\frac{11465}{N^{7}}-\frac{92777}{N^{8}}+\frac{1626151}{N^{9}}
\end{aligned}
$$

The numerical accuracy of the asymptotic representations at a given suitably large value of $N$ lowers with growing $k$, i.e., one has to choose larger values of $N$ correspondingly to apply the asymptotic formulae. The above expansions can easily be extended to higher inverse powers of $N$. The recursion for $\phi_{k}(l, k N)$ is given in (3.30), respectively, (3.35) to (3.46) in a more compact form. Due to these for any $N \in \mathbb{C}$ at which $\phi_{k}(l, N)$ is analytic, one may map $\phi_{k}(l, N)$ to values $|N| \gg 1, \arg (N)<\pi$ and use the asymptotic representations.

The single cyclotomic harmonic sums of higher weight obey the representations

$$
\begin{aligned}
& \sum_{k=0}^{N-1} \frac{1}{(l k+m)^{n}}=\frac{(-1)^{n-1}}{(n-1) !} \int_{0}^{1} d x \ln ^{n-1}(x) x^{m-1} \frac{x^{l N}-1}{x^{l}-1}, \quad m<l, \\
& \sum_{k=0}^{N-1} \frac{(-1)^{k}}{(l k+m)^{n}}=\frac{(-1)^{n}}{(n-1) !} \int_{0}^{1} d x \ln ^{n-1}(x) x^{m-1} \frac{\left(-x^{l}\right)^{N}-1}{x^{l}+1}, \quad m<l
\end{aligned}
$$

for $l, m, n \in \mathbb{N}_{+}$, which may be expressed in terms of cyclotomic letters again. We note that

$$
\frac{\partial^{n}}{\partial m^{n}} \int_{0}^{1} d x x^{m-1} f(x)=\int_{0}^{1} d x x^{m-1} \ln ^{n}(x) f(x) .
$$

Therefore, (4.42) and (4.43) can be expressed by the corresponding derivatives of $\phi_{k}(n, l N)$ for $N$ and corresponding constants. 


\section{B. Cyclotomic harmonic polylogarithms at $x=1$}

We consider the cyclotomic harmonic polylogarithm $C_{a, \vec{b}}^{c, \vec{d}}(x)$. Its value at $x=1$ is given by

$$
C_{a, \vec{b}}^{c, \vec{d}}(1)=\int_{0}^{1} d x f_{a}^{c}(x) C_{\vec{b}}^{\vec{d}}(x) .
$$

Let $f_{a}^{c}(x)=x^{l} / \Phi_{k}(x)$, with $l<\operatorname{deg}\left(\Phi_{k}(x)\right)$ and $n$ be the smallest integer such that $\Phi_{k}(x) \mid\left(x^{n}-1\right)$, with

$$
\frac{1}{\Phi_{k}(x)}=\frac{\prod_{j} \Phi_{j}(x)}{x^{n}-1}
$$

Since $x \in[0,1]$, the representation

$$
\frac{x^{l}}{\Phi_{k}(x)}=-x^{l} \prod_{k} \Phi_{k}(x) \sum_{j=0}^{\infty} x^{j n}=\sum_{m=0}^{w} a_{m}(l) x^{m} \sum_{j=0}^{\infty} x^{j n}, \quad a_{m}(l) \in \mathbb{Z}
$$

holds. Thus, we get

$$
\int_{0}^{1} d x \frac{x^{l}}{\Phi_{k}(x)}=\sum_{m=0}^{w} a_{m}(l) \sum_{j=0}^{\infty} \frac{1}{j n+m+1},
$$

representing the value of the depth $\mathrm{d}=1$ cyclotomic harmonic polylogarithms at $x=1$ as a linear combination of the corresponding infinite cyclotomic sums, see Sec. V

We return now to Eq. (4.45). Integration by parts yields

$$
C_{a, \vec{b}}^{c, \vec{d}}(1)=C_{a}^{c}(1) C_{\vec{b}}^{\vec{d}}(1)-\sum_{j=0}^{\infty} \sum_{m=0}^{w} a_{m} \int_{0}^{1} d x \frac{x^{j n+m+1}}{j n+m+1} C_{\vec{b}}^{\vec{d}}(x) .
$$

Since the Mellin transform of a cyclotomic harmonic polylogarithm can be represented by a linear combination of finite cyclotomic harmonic sums, the weighted infinite sum of the latter ones in (4.49) is thus given as a polynomial of infinite cyclotomic harmonic sums, Sec. V.

\section{Relations of Cyclotomic Harmonic Sums}

The cyclotomic harmonic sums obey differentiation relations, cf. Refs. 7 and 28, stuffle relations due to their quasi-shuffle algebra, cf. Ref. 9, and multiple argument relations. ${ }^{89}$

\section{Differentiation}

As has been illustrated in Sec. III, cyclotomic harmonic sums can be represented as linear combinations of Mellin transforms (3.27) of cyclotomic harmonic polylogarithms. Based on this representation, the differentiation of these sums is defined by

$$
\frac{\partial^{m}}{\partial N^{m}} S_{\left\{a_{1}, b_{1}, c_{1}\right\}, \ldots,\left\{a_{k}, b_{k}, c_{k}\right\}}(N)=\sum_{n=1}^{s} e_{n} \int_{0}^{1} d x x^{l N} l^{m} \ln ^{m}(x)\left(f_{\alpha_{n}}^{\beta_{n}}(x)\right)^{u_{n}} C_{\overrightarrow{\gamma_{n}}}^{\overrightarrow{\delta_{n}}}(x) .
$$

The product $\ln ^{m}(x) C_{\vec{\alpha}_{n}}^{\vec{\beta}_{n}}(x)=m ! C_{0, \ldots, 0}^{0, \ldots, 0} C_{\overrightarrow{\alpha_{n}}}^{\vec{\beta}_{n}}(x)$ with $m$ consecutive zeros may be transformed into a linear combination of cyclotomic harmonic polylogarithms using the shuffle relation (3.21). Finally, using the inverse Mellin transform, the derivative (4.50) of a cyclotomic harmonic sum with respect to $N$ is given as a polynomial expression in terms of cyclotomic harmonic sums and cyclotomic harmonic polylogarithms at $x=1$. Together with Sec. IV B, the derivative (4.50) can be expressed as a polynomial expression with rational coefficients in terms of cyclotomic harmonic sums and their values at $N \rightarrow \infty$. The corresponding relations are denoted by $(D)$. Further details on cyclotomic harmonic sums at infinity are given in Sec. V.

A given finite cyclotomic harmonic sum is determined for $N \in \mathbb{C}$ by its asymptotic representation and the corresponding recursion from $N \rightarrow(N-1)$. Both the asymptotic representation and the recursion can be easily differentiated analytically. Therefore, any differentiation of a cyclotomic harmonic sum with respect to $N$ is closely related to the original sum. For this reason one may 
collect these derivatives in classes

$$
S_{\left\{a_{1}, b_{1}, c_{1}\right\}, \ldots,\left\{a_{k}, b_{k}, c_{k}\right\}}^{(D)}(N)=\left\{\frac{\partial^{n}}{\partial N^{n}} S_{\left\{a_{1}, b_{1}, c_{1}\right\}, \ldots,\left\{a_{k}, b_{k}, c_{k}\right\}}(N) ; n \in \mathbb{N}\right\} .
$$

\section{Stuffle algebra}

To derive the stuffle relations, cf. Refs. 11 and 28, we consider the product of two denominator terms. For $a_{1}, a_{2}, b_{1}, b_{2}, c_{1}, c_{2}, i \in \mathbb{N}$ they are given by

$$
\begin{aligned}
\frac{1}{\left(a_{1} i+b_{1}\right)^{c_{1}}\left(a_{2} i+b_{2}\right)^{c_{2}}} & =(-1)^{c_{1}} \sum_{j=1}^{c_{1}}(-1)^{j}\left(\begin{array}{c}
c_{1}+c_{2}-j-1 \\
c_{2}-1
\end{array}\right) \frac{a_{1}^{c_{2}} a_{2}^{c_{1}-j}}{\left(a_{1} b_{2}-a_{2} b_{1}\right)^{c_{1}+c_{2}-j}} \frac{1}{\left(a_{1} i+b_{1}\right)^{j}} \\
& +(-1)^{c_{2}} \sum_{j=1}^{c_{2}}(-1)^{j}\left(\begin{array}{c}
c_{1}+c_{2}-j-1 \\
c_{1}-1
\end{array}\right) \frac{a_{1}^{c_{2}-j} a_{2}^{c_{1}}}{\left(a_{2} b_{1}-a_{1} b_{2}\right)^{c_{1}+c_{2}-j}} \frac{1}{\left(a_{2} i+b_{2}\right)^{j}}
\end{aligned}
$$

and

$$
\frac{1}{\left(a_{1} i+b_{1}\right)^{c_{1}}\left(a_{2} i+b_{2}\right)^{c_{2}}}=\left(\frac{a_{1}}{a_{2}}\right)^{c_{2}} \frac{1}{\left(a_{1} i+b_{1}\right)^{c_{1}+c_{2}}}
$$

if $a_{1} b_{2}=a_{2} b_{1}$.

The product of two cyclotomic harmonic sums has the following representation. Let $a_{i}, b_{i}, d_{i}, e_{i}, k, l, n \in \mathbb{N}_{+}$and $c_{i}, f_{i} \in \mathbb{Z} \backslash\{0\}$. If $a_{1} e_{1} \neq d_{1} b_{1}$, one has

$$
\begin{aligned}
& S_{\left\{a_{1}, b_{1}, c_{1}\right\}, \ldots,\left\{a_{k}, b_{k}, c_{k}\right\}}(n) S_{\left\{d_{1}, e_{1}, f_{1}\right\}, \ldots,\left\{d_{l}, e_{l}, f_{l}\right\}}(n)= \\
& \sum_{i=1}^{n} \frac{\operatorname{sign}\left(c_{1}\right)^{i}}{\left(a_{1} i+b_{1}\right)^{\left|c_{1}\right|}} S_{\left\{a_{2}, b_{2}, c_{2}\right\}, \ldots,\left\{a_{k}, b_{k}, c_{k}\right\}}(i) S_{\left\{d_{1}, e_{1}, f_{1}\right\}, \ldots,\left\{d_{l}, e_{l}, f_{l}\right\}}(i) \\
& +\sum_{i=1}^{n} \frac{\operatorname{sign}\left(f_{1}\right)^{i}}{\left(d_{1} i+e_{1}\right)^{\left|f_{1}\right|}} S_{\left\{a_{1}, b_{1}, c_{1}\right\}, \ldots,\left\{a_{k}, b_{k}, c_{k}\right\}}(i) S_{\left\{d_{2}, e_{2}, f_{2}\right\}, \ldots,\left\{d_{l}, e_{l}, f_{l}\right\}}(i) \\
& -\sum_{i=1}^{n}\left((-1)^{\left|c_{1}\right|} \sum_{j_{1}=1}^{\left|c_{1}\right|}(-1)^{j}\left(\begin{array}{c}
\left|c_{1}\right|+\left|f_{1}\right|-j-1 \\
\left|f_{1}\right|-1
\end{array}\right) \frac{a_{1}^{\left|f_{1}\right|} d_{1}^{\left|c_{1}\right|-j}}{a_{1} e_{1}-d_{1} b_{1}} \frac{1}{\left(a_{1} i+b_{1}\right)^{j}}\right. \\
& \left.+(-1)^{\left|f_{1}\right|} \sum_{j=1}^{\left|f_{1}\right|}(-1)^{j}\left(\begin{array}{c}
\left|c_{1}\right|+\left|f_{1}\right|-j-1 \\
\left|f_{1}\right|-1
\end{array}\right) \frac{a_{1}^{\left|f_{1}\right|-j} d_{1}^{\left|c_{1}\right|}}{d_{1} b_{1}-a_{1} e_{1}} \frac{1}{\left(d_{1} i+e_{1}\right)^{j}}\right) \\
& \times S_{\left\{a_{2}, b_{2}, c_{2}\right\}, \ldots,\left\{a_{k}, b_{k}, c_{k}\right\}}(i) S_{\left\{d_{2}, e_{2}, f_{2}\right\}, \ldots,\left\{d_{l}, e_{l}, f_{l}\right\}}(i),
\end{aligned}
$$

respectively, for $a_{1} e_{1}=d_{1} b_{1}$, one has

$$
\begin{aligned}
& S_{\left\{a_{1}, b_{1}, c_{1}\right\}, \ldots,\left\{a_{k}, b_{k}, c_{k}\right\}}(n) S_{\left\{d_{1}, e_{1}, f_{1}\right\}, \ldots,\left\{d_{l}, e_{l}, f_{l}\right\}}(n)= \\
& \sum_{i=1}^{n} \frac{\operatorname{sign}\left(c_{1}\right)^{i}}{\left(a_{1} i+b_{1}\right)^{\left|c_{1}\right|}} S_{\left\{a_{2}, b_{2}, c_{2}\right\}, \ldots,\left\{a_{k}, b_{k}, c_{k}\right\}}(i) S_{\left\{d_{1}, e_{1}, f_{1}\right\}, \ldots,\left\{d_{l}, e_{l}, f_{l}\right\}}(i) \\
&+ \sum_{i=1}^{n} \frac{\operatorname{sign}\left(f_{1}\right)^{i}}{\left(d_{1} i+e_{1}\right)^{\left|f f_{1}\right|}} S_{\left\{a_{1}, b_{1}, c_{1}\right\}, \ldots,\left\{a_{k}, b_{k}, c_{k}\right\}}(i) S_{\left\{d_{2}, e_{2}, f_{2}\right\}, \ldots,\left\{d_{l}, e_{l}, f_{l}\right\}}(i) \\
&-\left(\frac{a_{1}}{d_{1}}\right)^{\left|f_{1}\right|} \sum_{i=1}^{n} \frac{\left(\operatorname{sign}\left(c_{1}\right) \operatorname{sign}\left(f_{1}\right)\right)^{i}}{\left(a_{1} i+b_{1}\right)^{\left|c_{1}\right|+\left|f_{1}\right|}} \\
& \times S_{\left\{a_{2}, b_{2}, c_{2}\right\}, \ldots,\left\{a_{k}, b_{k}, c_{k}\right\}}(i) S_{\left\{d_{2}, e_{2}, f_{2}\right\}, \ldots,\left\{d_{l}, e_{l}, f_{l}\right\}}(i) .
\end{aligned}
$$

Subsequently, the relations given by (4.54) and (4.55) are denoted by $(A)$. 


\section{Synchronization}

A first multiple argument relation is implied as follows. Let $a, b, k \in \mathbb{N}, c \in \mathbb{Z} \backslash\{0\}, k \geq 2$. Then

$$
S_{\{a, b, c\}}(k \cdot N)=\sum_{i=0}^{k-1} \operatorname{sign}(c)^{i} S_{\left\{k \cdot a, b-a \cdot i, \operatorname{sign}(c)^{k}|c|\right\}}(N) .
$$

For $a_{i}, b_{i}, m, k \in \mathbb{N}, c_{i} \in \mathbb{Z} \backslash\{0\}, k \geq 2$, the general cyclotomic harmonic sums obey

$$
\begin{aligned}
S_{\left\{a_{m}, b_{m}, c_{m}\right\},\left\{a_{m-1}, b_{m-1}, c_{m-1}\right\}, \ldots,\left\{a_{1}, b_{1}, c_{1}\right\}}(k \cdot N) & \\
= & \sum_{i=0}^{m-1} \sum_{j=1}^{N} \frac{S_{\left\{a_{m-1}, b_{m-1}, c_{m-1}\right\}, \ldots,\left\{a_{1}, b_{1}, c_{1}\right\}}(k \cdot j-i) \operatorname{sign}\left(c_{m}\right)^{k \cdot j-i}}{\left(a_{m}(k \cdot j-i)+b_{1}\right)^{\left|c_{m}\right|}} .
\end{aligned}
$$

Repeated application of (4.56) and (4.57) allows to represent cyclotomic harmonic sums of argument $k n$ by those of argument $n$. Subsequently, the resulting relations are denoted by $(M)$.

\section{Duplication relations}

The usual duplication relation ${ }^{11}$ holds also for cyclotomic harmonic sums:

$$
\sum_{ \pm} S_{\left\{a_{m}, b_{m}, \pm c_{m}\right\},\left\{a_{m-1}, b_{m-1}, \pm c_{m-1}\right\}, \ldots,\left\{a_{1}, b_{1}, \pm c_{1}\right\}}(2 N)=2^{m} S_{\left\{2 a_{m}, b_{m}, c_{m}\right\}, \ldots,\left\{2 a_{1}, b_{1}, c_{1}\right\}}(N) .
$$

The proof of (4.58) is given in Appendix B.

Similar to (4.58) one obtains a second duplication relation:

$$
\begin{aligned}
\sum_{d_{i} \in\{-1,1\}} d_{m} d_{m-1} \cdots d_{1} S_{\left\{a_{m}, b_{m}, d_{m} c_{m}\right\},\left\{a_{m-1}, b_{m-1}, d_{m-1} c_{m-1}\right\}, \ldots,\left\{a_{1}, b_{1}, d_{1} c_{1}\right\}}(2 N) & \\
& =2^{m} S_{\left\{2 a_{m}, b_{m}-a_{m}, c_{m}\right\}, \ldots,\left\{2 a_{1}, b_{1}-a_{1}, c_{1}\right\}}(N) ;
\end{aligned}
$$

its proof is given in Appendix B. The resulting algebraic relations of (4.58) and (4.59) are denoted by $\left(H_{1}\right)$ and $\left(H_{2}\right)$, respectively. We remark that more general relations arise for generalized cyclotomic harmonic sums presented in Sec. VI, (6.12) and (6.13).

\section{Sums of higher depth and weight}

As an example we consider the class of cyclotomic sums, which occur in the physical application mentioned in Sec. I. They are given by iteration of the summands

$$
\frac{1}{k^{l_{1}}}, \quad \frac{(-1)^{k}}{k^{l_{2}}}, \quad \frac{1}{(2 k+1)^{l_{3}}}, \quad \frac{(-1)^{k}}{(2 k+1)^{l_{4}}}
$$

with $N \geq k \geq 1, l_{i}, k \in \mathbb{N}_{+}$. As pointed out earlier, this class of cyclotomic harmonic sums can be expressed by Mellin transforms in terms of cyclotomic harmonic polylogarithms generated by the alphabet (3.29).

We apply the relations in Sec. IV B to derive the corresponding bases for a given weight $\mathbf{w}$. The cyclotomic harmonic sums can be represented over the corresponding bases. Applying the relations using computer algebra we find the pattern for the number of basis elements up to $w=5$ given in Table II.

Due to the arguments given in Sec. IV C 1 above, for any occurrence of a differential operator $\left(\partial^{m} / \partial N^{m}\right) S_{a_{1}, b_{1}, c_{1}}, \ldots, a_{k}, b_{k}, c_{k}(N)$, only one representative is counted.

The total number of sums of weight $\mathrm{w}$ for the alphabet (4.60) is

$$
N_{S}(w)=4 \cdot 5^{w-1} \text {. }
$$


TABLE II. Reduction of the number of cyclotomic harmonic sums $N_{S}$ over the elements (4.60) at given weight $w$ by applying the three multiple argument relations $\left(H_{1}, H_{2}, M\right)$, differentiation with respect to the external sum index $N,(D)$, and the algebraic relations $(A)$. A sequence of symbols corresponds to the combination of these relations.

\begin{tabular}{rrrrrrrrrrrr}
\hline \hline $\mathrm{w}$ & \multicolumn{1}{c}{$N_{S}$} & $H_{1}$ & $H_{1}, H_{2}$ & $H_{1}, M$ & $H_{1}, H_{2}, M$ & $D$ & $H_{1}, H_{2}, M, D$ & $A$ & $H_{1}, H_{2}, M, A$ & $A, D$ & All \\
\hline 1 & 4 & 3 & 3 & 2 & 2 & 4 & 2 & 4 & 2 & 4 & 2 \\
2 & 20 & 18 & 17 & 16 & 15 & 16 & 13 & 10 & 8 & 6 & 6 \\
3 & 100 & 96 & 93 & 92 & 89 & 80 & 74 & 40 & 35 & 30 & 27 \\
4 & 500 & 492 & 485 & 484 & 477 & 400 & 388 & 150 & 142 & 110 & 107 \\
5 & 2500 & 2484 & 2469 & 2468 & 2453 & 2000 & 1976 & 624 & 607 & 474 & 465 \\
\hline \hline
\end{tabular}

We mention that the single application of any of the multiple argument relations $\left(M, H_{1}, H_{2}\right)$ leads to the same number of basis sums:

$$
N_{H_{1}}=N_{H_{2}}=N_{M} .
$$

This also applies to the combinations $H_{1} M$ and $H_{2} M$ :

$$
N_{H_{1}, M}=N_{H_{2}, M} .
$$

Explicit counting relations for the number of basis elements given Table II can be derived:

$$
\begin{aligned}
N_{A}(w) & =\frac{1}{w} \sum_{d \mid w} \mu\left(\frac{w}{d}\right) 5^{d}, \\
N_{D}(w) & =N_{S}(w)-N_{S}(w-1)=16 \cdot 5^{w-2}, \\
N_{H_{1}}(w) & =N_{S}(w)-2^{w-1}=4 \cdot 5^{w-1}-2^{w-1}, \\
N_{H_{1} H_{2}}(w) & =N_{S}(w)-\left(2 \cdot 2^{w-1}-1\right)=4 \cdot 5^{w-1}-\left(2 \cdot 2^{w-1}-1\right), \\
N_{H_{1} M}(w) & =N_{S}(w)-2 \cdot 2^{w-1}=4 \cdot 5^{w-1}-2 \cdot 2^{w-1}, \\
N_{H_{1} H_{2} M}(w) & =N_{S}(w)-\left(3 \cdot 2^{w-1}-1\right)=4 \cdot 5^{w-1}-\left(3 \cdot 2^{w-1}-1\right), \\
N_{A D}(w) & =N_{A}(w)-N_{A}(w-1) \\
& =\frac{1}{w} \sum_{d \mid w} \mu\left(\frac{w}{d}\right) 5^{d}-\frac{1}{w-1} \sum_{d \mid w-1} \mu\left(\frac{w-1}{d}\right) 5^{d}, \\
N_{A D H_{1} H_{2} M}(w) & =N_{A H_{1} H_{2} M}(w)-N_{A H_{1} H_{2} M}(w-1), \\
N_{D H_{1} H_{2} M}(w) & =N_{H_{1} H_{2} M}(w)-N_{H_{1} H_{2} M}(w-1)=16 \cdot 5^{w-2}-3 \cdot 2^{w-2}, \\
N_{A H_{1} H_{2} M}(w) & =\frac{1}{w} \sum_{d \mid w} \mu\left(\frac{w}{d}\right) 5^{d}-\left(3 \cdot \frac{1}{w} \sum_{d \mid w} \mu\left(\frac{w}{d}\right) 2^{d}-1\right),
\end{aligned}
$$




$$
=\frac{1}{w} \sum_{d \mid w} \mu\left(\frac{w}{d}\right)\left(5^{d}-3 \cdot 2^{d}\right)-\frac{1}{w-1} \sum_{d \mid w-1} \mu\left(\frac{w-1}{d}\right)\left(5^{d}-3 \cdot 2^{d}\right) .
$$

Here, $\mu$ denotes the Möbius function. ${ }^{43}$

The analytic continuation of the cyclotomic harmonic sums can be performed as outlined for the case of the single sums in Sec. IV A. Their representation as a Mellin transform of the cyclotomic harmonic polylogarithms (3.26) relates them to factorial series, except the case $c_{1}=1, s_{1}=1$, cf. (2.1). If a sequential set of first indices $c_{i}=1, s_{i}=1$ occurs, one may reduce the corresponding sum algebraically to convergent sums, separating factors, cf. Ref. 6 . That is, also in the general case the poles of the cyclotomic harmonic sums are located at $-k, k \in \mathbb{N}$. The recursion relations of the cyclotomic harmonic sums (2.1) imply the shift relations $N \rightarrow N+1$ in a hierarchic manner, referring to the sums of lower depth.

To accomplish the analytic continuation in $N$, the asymptotic representations of the cyclotomic harmonic sums have to be computed. Since the cyclotomic harmonic sums are represented over respective bases, only the asymptotic representations for the basis elements have to be derived. One way consists in using iterated integration by parts,

$$
\int_{0}^{1} d x x^{N} f(x)=\frac{f(1)}{N+1}-\frac{1}{N+1} \int_{0}^{1} d x x^{N}\left[x f^{\prime}(x)\right]
$$

with $f(x)$ being a linear combination of cyclotomic polylogarithms. Here, $f(x)$ is conveniently expressed in a power series to which we turn now.

We illustrate the principle steps considering the alphabet (3.29) related to (4.60), cf. (3.16) and (3.17). In general, the cyclotomic harmonic polylogarithms $C_{\vec{a}} \vec{b}(x)$ over the alphabet (3.29) do not have a regular Taylor series expansion, cf. Ref. 26. This is due to the effect that trailing zeroes in the index set may cause powers of $\ln (x)$. Hence, the proper expansion is one in terms of both $x$ and $\ln (x)$. For depth and $0<x<1$, one obtains

$$
\begin{aligned}
& C_{0}^{0}(x)=\ln (x), \\
& C_{2}^{0}(x)=-\sum_{i=1}^{\infty} \frac{(-x)^{i}}{i}=-\sum_{i=1}^{\infty} \frac{(-x)^{2 i}}{2 i}+\sum_{i=1}^{\infty} \frac{(-x)^{2 i+1}}{2 i+1}, \\
& C_{1}^{0}(x)=-\sum_{i=1}^{\infty} \frac{x^{i}}{i}=\sum_{i=1}^{\infty} \frac{(-x)^{2 i}}{2 i}+\sum_{i=1}^{\infty} \frac{(-x)^{2 i+1}}{2 i+1}, \\
& C_{4}^{0}(x)=-\sum_{i=1}^{\infty} \frac{(-1)^{i} x^{2 i-1}}{2 i-1}, \\
& C_{4}^{1}(x)=\sum_{i=1}^{\infty} \frac{(-1)^{i} x^{2 i}}{2 i} .
\end{aligned}
$$

Let $C_{\vec{a}} \vec{b}(x)$ be a cyclotomic harmonic polylogarithm with depth $d$. Assume that its power series expansion is of the form

$$
C_{\vec{a}}^{\vec{b}}(x)=\sum_{j=1}^{w} \sum_{i=1}^{\infty} \frac{\sigma^{i} x^{2 i+c_{j}}}{\left(2 i+c_{j}\right)^{a}} S_{\vec{n}_{j}}(i)
$$

with the index sets $\vec{a}$ and $\vec{b}$ according to the iteration of the letters (4.76) to (4.80), and $\vec{n}_{j}$ being a corresponding index set of the cyclotomic harmonic sum, $x \in(0,1), w \in \mathbb{N}$, and $c_{j} \in \mathbb{Z}$. 
Then the expansion of the cyclotomic harmonic polylogarithms of depth $d+1$ is obtained by using

$$
\begin{gathered}
C_{0, \vec{a}}^{0, \vec{b}}(x)=\sum_{j=1}^{w} \sum_{i=1}^{\infty} \frac{\sigma^{i} x^{2 i+c_{j}}}{\left(2 i+c_{j}\right)^{a+1}} S_{\vec{n}_{j}}(i), \\
C_{0, \vec{a}}^{1, \vec{b}}(x)=\sum_{j=1}^{w} \sum_{i=1}^{\infty} \frac{x^{2 i+c_{j}+1}}{\left(2 i+c_{j}+1\right)} S_{\left\{2, c_{j}, \sigma a\right\}, \vec{n}_{j}}(i)+\sum_{j=1}^{w} \sum_{i=1}^{\infty} \frac{x^{2 i+c_{j}+2}}{\left(2 i+c_{j}+2\right)} S_{\left\{2, c_{j}, \sigma a\right\}, \vec{n}_{j}}(i), \\
C_{2, \vec{a}}^{0, \vec{b}}(x)=\sum_{j=1}^{w} \sum_{i=1}^{\infty} \frac{x^{2 i+c_{j}+1}}{\left(2 i+c_{j}+1\right)} S_{\left\{2, c_{j}, \sigma a\right\}, \vec{n}_{j}}(i)-\sum_{j=1}^{w} \sum_{i=1}^{\infty} \frac{x^{2 i+c_{j}+2}}{\left(2 i+c_{j}+2\right)} S_{\left\{2, c_{j}, \sigma a\right\}, \vec{n}_{j}}(i), \\
C_{4, \vec{a}}^{0, \vec{b}}(x)=\sum_{j=1}^{w} \sum_{i=1}^{\infty} \frac{(-1)^{i} x^{2 i+c_{j}+1}}{\left(2 i+c_{j}+1\right)} S_{\left\{2, c_{j},-\sigma a\right\}, \vec{n}_{j}}(i), \\
C_{4, \vec{m}}^{1, \vec{m}}(x)=\sum_{j=1}^{w} \sum_{i=1}^{\infty} \frac{(-1)^{i} x^{2 i+c_{j}+2}}{\left(2 i+c_{j}+2\right)} S_{\left\{2, c_{j},-\sigma a\right\}, \vec{n}_{j}}(i) .
\end{gathered}
$$

Sample proofs of these relations are given in Appendix B.

The analytic continuation of the cyclotomic harmonic sums at larger depths to $N \in \mathbb{C}$ is performed analogously to the case discussed in Sec. IV A. Shifts parallel to the real axis are performed with the recurrence relation induced by (2.1). The asymptotic relations for $N \rightarrow \infty,|\arg (N)|<\pi$ can be derived analytically to arbitrary precision. Examples are

$$
\begin{aligned}
& S_{\{2,1,2\},\{1,0,-2\}}(N) \sim 4 C_{f_{4}^{0}, f_{0}^{0}}(1)^{2}+4 C_{f_{4}^{0}}(1) C_{f_{0}^{0}, f_{4}^{0}, f_{0}^{0}}(1)-4 C_{f_{4}^{0}, f_{0}^{0}, f_{4}^{0}, f_{0}^{0}}(1)+\left(\frac{\pi^{2}}{2}-\frac{1}{N}\right. \\
& +\frac{1}{N^{2}}-\frac{11}{12 N^{3}}+\frac{3}{4 N^{4}}-\frac{127}{240 N^{5}}+\frac{5}{16 N^{6}}-\frac{221}{1344 N^{7}}+\frac{7}{64 N^{8}} \\
& \left.-\frac{367}{3840 N^{9}}+\frac{9}{256 N^{10}}\right) C_{f_{4}^{1}, f_{0}^{0}}(1)+(-1)^{N}\left(\frac{1}{16 N^{4}}-\frac{1}{4 N^{5}}+\frac{27}{64 N^{6}}\right. \\
& \left.-\frac{1}{32 N^{7}}-\frac{269}{256 N^{8}}-\frac{11}{32 N^{9}}+\frac{8699}{1024 N^{10}}\right)+O\left(\frac{1}{N^{11}}\right) \\
& S_{\{2,1,2\},\{2,1,-2\}}(N) \sim-\frac{\pi^{2}}{8}+C_{f_{4}^{0}}(1) C_{f_{0}^{0}, f_{4}^{1}, f_{0}^{0}}(1)-C_{f_{4}^{0}, f_{0}^{0}, f_{4}^{1}, f_{0}^{0}}(1)+\frac{1}{4 N}-\frac{1}{4 N^{2}}+\frac{11}{48 N^{3}} \\
& -\frac{3}{16 N^{4}}+\frac{127}{960 N^{5}}-\frac{5}{64 N^{6}}+\frac{221}{5376 N^{7}}-\frac{7}{256 N^{8}}+\frac{367}{15360 N^{9}} \\
& -\frac{9}{1024 N^{10}}+(-1)^{N}\left(\frac{1}{64 N^{4}}-\frac{5}{64 N^{5}}+\frac{25}{128 N^{6}}-\frac{61}{256 N^{7}}\right.
\end{aligned}
$$




$$
\begin{aligned}
& \left.-\frac{77}{1024 N^{8}}+\frac{221}{512 N^{9}}+\frac{1545}{1024 N^{10}}\right)+C_{f_{4}^{0}, f_{0}^{0}}(1)\left(C_{4}, f_{0}^{0}(1)-\frac{\pi^{2}}{8}\right. \\
& +\frac{1}{4 N}-\frac{1}{4 N^{2}}+\frac{11}{48 N^{3}}-\frac{3}{16 N^{4}}+\frac{127}{960 N^{5}}-\frac{5}{64 N^{6}}+\frac{221}{5376 N^{7}} \\
& \left.-\frac{7}{256 N^{8}}+\frac{367}{15360 N^{9}}-\frac{9}{1024 N^{10}}\right)+O\left(\frac{1}{N^{11}}\right) .
\end{aligned}
$$

Given a cyclotomic harmonic sum with the iterative denominators (4.60) and given the number of desired terms, the corresponding expansion can be computed on demand by the HARMONICSUMS package.

\section{SPECIAL VALUES}

The values of the cyclotomic harmonic polylogarithms at argument $x=1$ and, related to it, the associated cyclotomic harmonic sums at $N \rightarrow \infty$ occur in various relations of the finite cyclotomic harmonic sums and the Mellin transforms of cyclotomic harmonic polylogarithms. In this section we investigate their relations and basis representations. The infinite cyclotomic harmonic sums extend the Euler-Zagier and multiple zeta values ${ }^{11}$ and are related at lower weight and depth to other known special numbers. We first consider the single non-alternating and alternating sums up to cyclotomy $\mathrm{I}=6$ at general weight $\mathrm{w}$. Next, the relations of the infinite cyclotomic harmonic sums associated to the summands (4.60) up to weight $\mathbf{w}=6$ are worked out. Finally, we investigate the sums of weight $\mathrm{w}=1$ and 2 up to cyclotomy $\mathrm{I}=20$.

\section{A. Single infinite sums}

We consider the single sums of the type

$$
\sum_{k=0}^{\infty} \frac{( \pm 1)^{k}}{(l k+m)^{n}}
$$

with $l, m, n \in \mathbb{N}_{+}, l>m, n \geq 1$. These sums are linearly related to the colored harmonic sums by

$$
\sum_{k=1}^{\infty} \frac{e_{l}^{k}}{k^{n}}=\sum_{m=1}^{l} e_{l}^{m} \sum_{k=0}^{\infty} \frac{1}{(l k+m)^{n}}, \quad e_{l}=\exp \left(\frac{2 \pi i}{l}\right),
$$

and similar linear relations for the nested sums. We will address the latter sums in Sec. VI

\section{Non-alternating single sums at $\mathrm{w}=1$}

For the non-alternating sums, one obtains

$$
\sum_{k=0}^{\infty}\left[\frac{1}{l k+m}-\frac{1}{l k}\right]=-\frac{1}{l}\left[\gamma_{E}+\psi\left(\frac{m}{l}\right)\right]
$$

and

$$
\sigma_{0}=\sum_{k=1}^{\infty} 1 / k
$$

denoting the divergence used to regularize the first addend in the rhs of (5.3). The corresponding sums, except $\sigma_{0}$, can always be regularized. 

implies

The reflection symmetry of the $\psi$-function interchanging the arguments $x$ and $(1-x)$ (Ref. 36)

$$
\sum_{k=0}^{\infty}\left[\frac{1}{n k+l}-\frac{1}{n k+(n-l)}\right]=\frac{\pi}{n} \cot \left(\frac{l}{n} \pi\right) .
$$

The digamma-function at positive rational arguments obeys ${ }^{36,47}$

$$
\begin{gathered}
\psi\left(\frac{p}{q}\right)=-\gamma_{E}-\ln (2 q)-\frac{\pi}{2} \cot \left(\frac{p \pi}{q}\right)+2 \sum_{k=1}^{[(q-1) / 2]} \cos \left(\frac{2 \pi k p}{q}\right) \ln \left[\sin \left(\frac{\pi k}{q}\right)\right], \\
\psi\left(\frac{1}{n}\right)=-n\left(\gamma_{E}+\ln (n)\right)-\sum_{k=2}^{n} \psi\left(\frac{k}{n}\right) .
\end{gathered}
$$

Equation (5.6) is used to remove dependencies in (5.3) and (5.5). If the regular $q$-polygon is constructible, the trigonometric functions in (5.6) are algebraic numbers. This is the case for

$$
q \in\{2,3,4,5,6,8,10,12,15,16,17,20,24,30,32,34,40,48,51,60,64, \ldots\},
$$

Refs. 48 and 90. Due to (5.6) the constants $\ln \left(d_{i}\right)$ with $d_{i} \neq 1$, the divisors of $q$, and logarithms of further algebraic numbers will occur. These are all transcendental numbers. ${ }^{50}$

Let us list the first few of these relations, see also Ref. 51:

$$
\begin{aligned}
& -\frac{1}{2}\left[\gamma_{E}+\psi\left(\frac{1}{2}\right)\right]=\ln (2) \\
& -\frac{1}{3}\left[\gamma_{E}+\psi\left(\frac{1}{3}\right)\right]=\frac{1}{2} \ln (3)+\frac{\pi}{18} \sqrt{3} \\
& -\frac{1}{3}\left[\gamma_{E}+\psi\left(\frac{2}{3}\right)\right]=\frac{1}{2} \ln (3)-\frac{\pi}{18} \sqrt{3} \\
& -\frac{1}{4}\left[\gamma_{E}+\psi\left(\frac{1}{4}\right)\right]=\frac{3}{4} \ln (2)+\frac{\pi}{8} \\
& -\frac{1}{4}\left[\gamma_{E}+\psi\left(\frac{3}{4}\right)\right]=\frac{3}{4} \ln (2)-\frac{\pi}{8} \\
& -\frac{1}{5}\left[\gamma_{E}+\psi\left(\frac{1}{5}\right)\right]=\frac{\sqrt{5}}{10}[\ln (2)-\ln (\sqrt{5}-1)]+\frac{1}{4} \ln (5)+\frac{\sqrt{25+10 \sqrt{5}}}{50} \pi \\
& -\frac{1}{5}\left[\gamma_{E}+\psi\left(\frac{2}{5}\right)\right]=-\frac{\sqrt{5}}{10}[\ln (2)-\ln (\sqrt{5}-1)]+\frac{1}{4} \ln (5)+\frac{\sqrt{10-2 \sqrt{5}}}{40}\left[1-\frac{\sqrt{5}}{5}\right] \pi \\
& -\frac{1}{5}\left[\gamma_{E}+\psi\left(\frac{3}{5}\right)\right]=-\frac{\sqrt{5}}{10}[\ln (2)-\ln (\sqrt{5}-1)]+\frac{1}{4} \ln (5)-\frac{\sqrt{10-2 \sqrt{5}}}{40}\left[1-\frac{\sqrt{5}}{5}\right] \pi \\
& -\frac{4}{25+10 \sqrt{5}} \\
& -
\end{aligned}
$$




$$
\begin{aligned}
& -\frac{1}{6}\left[\gamma_{E}+\psi\left(\frac{1}{6}\right)\right]=\frac{1}{3} \ln (2)+\frac{1}{4} \ln (3)+\frac{\pi}{12} \sqrt{3}, \\
& -\frac{1}{6}\left[\gamma_{E}+\psi\left(\frac{5}{6}\right)\right]=\frac{1}{3} \ln (2)+\frac{1}{4} \ln (3)-\frac{\pi}{12} \sqrt{3}, \quad \text { etc. }
\end{aligned}
$$

\section{Alternating single sums at $\mathrm{w}=1$}

The alternating sums at $\mathbf{w}=1$ have the representation

$$
\sum_{k=0}^{\infty} \frac{(-1)^{k}}{l k+m}=\frac{1}{2 l}\left[\psi\left(\frac{m+l}{2 l}\right)-\psi\left(\frac{m}{2 l}\right)\right]
$$

with the reflection relation

$$
\sum_{k=0}^{\infty}\left[\frac{(-1)^{k}}{n k+l}-\frac{(-1)^{k}}{n k+(n-l)}\right]=\frac{1}{2 n}\left[\psi\left(\frac{1}{2}-\frac{l}{2 n}\right)+\psi\left(\frac{1}{2}+\frac{l}{2 n}\right)-\psi\left(1-\frac{l}{2 n}\right)-\psi\left(\frac{l}{2 n}\right)\right] .
$$

For the sums at $\mathbf{w}=1$, one obtains

$$
\begin{aligned}
& \sum_{k=0}^{\infty} \frac{(-1)^{k}}{2 k+1}=\frac{\pi}{4} \\
& \sum_{k=0}^{\infty} \frac{(-1)^{k}}{3 k+1}=\frac{1}{3}\left[\frac{\pi}{\sqrt{3}}+\ln (2)\right], \\
& \sum_{k=0}^{\infty} \frac{(-1)^{k}}{3 k+2}=\frac{1}{3}\left[\frac{\pi}{\sqrt{3}}-\ln (2)\right], \\
& \sum_{k=0}^{\infty} \frac{(-1)^{k}}{4 k+1}=\frac{1}{2 \sqrt{2}}\left[\frac{\pi}{2}-\ln (\sqrt{2}-1)\right], \\
& \sum_{k=0}^{\infty} \frac{(-1)^{k}}{4 k+3}=\frac{1}{2 \sqrt{2}}\left[\frac{\pi}{2}+\ln (\sqrt{2}-1)\right], \\
& \sum_{k=0}^{\infty} \frac{(-1)^{k}}{5 k+1}=\frac{1}{5}[1+\sqrt{5}] \ln (2)-\frac{1}{\sqrt{5}} \ln (\sqrt{5}-1)+\frac{1+\sqrt{5}}{5 \sqrt{10+2 \sqrt{5}}} \pi \\
& \sum_{k=0}^{\infty} \frac{(-1)^{k}}{5 k+2}=-\frac{1}{5}[1-\sqrt{5}] \ln (2)-\frac{1}{\sqrt{5}} \ln (\sqrt{5}-1)+\frac{\sqrt{5}-1}{5 \sqrt{10-2 \sqrt{5}}} \pi \\
& \sum_{k=0}^{\infty} \frac{(-1)^{k}}{5 k+3}=\frac{1}{5}[1-\sqrt{5}] \ln (2)+\frac{1}{\sqrt{5}} \ln (\sqrt{5}-1)+\frac{\sqrt{5}-1}{5 \sqrt{10-2 \sqrt{5}}} \pi \\
& \sum_{k=0}^{\infty} \frac{(-1)^{k}}{5 k+4}=-\frac{1}{5}[1+\sqrt{5}] \ln (2)+\frac{1}{\sqrt{5}} \ln (\sqrt{5}-1)+\frac{1+\sqrt{5}}{5 \sqrt{10+2 \sqrt{5}}} \pi
\end{aligned}
$$




$$
\begin{aligned}
& \sum_{k=0}^{\infty} \frac{(-1)^{k}}{6 k+1}=\frac{\pi}{6}+\frac{1}{\sqrt{3}}\left[\frac{1}{2} \ln (2)-\ln (\sqrt{3}-1)\right], \\
& \sum_{k=0}^{\infty} \frac{(-1)^{k}}{6 k+5}=\frac{\pi}{6}-\frac{1}{\sqrt{3}}\left[\frac{1}{2} \ln (2)-\ln (\sqrt{3}-1)\right], \text { etc. }
\end{aligned}
$$

\section{Single infinite sums of higher weight}

These sums obey representations which are obtained by repeated differentiation of (5.3) and (5.20) for $m$ :

$$
\begin{aligned}
& \sum_{k=0}^{\infty} \frac{1}{(l k+m)^{n}}=\frac{1}{l^{n}} \zeta_{\mathrm{H}}\left(n, \frac{m}{l}\right)=\frac{1}{\Gamma(n)}\left(-\frac{1}{l}\right)^{n} \psi^{(n-1)}\left(\frac{m}{l}\right) \\
& \sum_{k=0}^{\infty} \frac{(-1)^{k}}{(l k+m)^{n}}=\frac{(-1)^{n-1}}{\Gamma(n)} \frac{1}{(2 l)^{n}}\left[\psi^{(n-1)}\left(\frac{m+l}{2 l}\right)-\psi^{(n-1)}\left(\frac{m}{2 l}\right)\right] .
\end{aligned}
$$

Here, $\zeta_{\mathrm{H}}$ is the Hurwitz $\zeta$-function ${ }^{52}$ with the serial representation

$$
\zeta_{\mathrm{H}}(s, a)=\sum_{n=0}^{\infty} \frac{1}{(n+a)^{s}} .
$$

We consider the representations of the polygamma functions at rational arguments for $p / q, p \nmid$ $q, q \in \mathbb{N}_{+}, q \leq 12 .{ }^{91}$ One obtains

$$
\psi^{(l)}\left(\frac{1}{2}\right)=(-1)^{l+1} l !\left(2^{l+1}-1\right) \zeta_{l+1}
$$

with

$$
\zeta_{2 n}=(-1)^{n-1} \frac{(2 \pi)^{2 n}}{2(2 n) !} B_{2 n},
$$

where $B_{n}$ denote the Bernoulli numbers. ${ }^{54,55}$ They are generated by

$$
\frac{x}{\exp (x)-1}=\sum_{n=0}^{\infty} \frac{B_{n}}{n !} x^{n}, \quad B_{2 n+1}=0 \text { for } n>1 .
$$

For odd values of $l$ no new basis elements occur due to (5.36).

The reflection formula ${ }^{36}$

$$
\psi^{(n)}(1-z)=(-1)^{n}\left[\psi^{n}(z)+\pi \frac{d^{n}}{d z^{n}} \cot (\pi z)\right]
$$

implies the relations for the argument $p / q$ and $(q-p) / q$. Likewise, the multiplication formula for the Hurwitz zeta function ${ }^{52}$ holds,

$$
\begin{gathered}
\zeta_{\mathrm{H}}(s, k z)=\frac{1}{k^{s}} \sum_{n=0}^{k-1} \zeta_{\mathrm{H}}\left(s, z+\frac{n}{k}\right), \quad k \in \mathbb{N}_{+}, \\
m^{l+1} \psi^{(l)}(m z)=\sum_{k=0}^{m-1} \psi^{(l)}\left(z+\frac{k}{m}\right)
\end{gathered}
$$

with the special relation

$$
n !(-1)^{n+1} \zeta_{n+1}\left[m^{n+1}-1\right]=\sum_{k=1}^{m-1} \psi^{(n)}\left(\frac{k}{m}\right) .
$$


One obtains, cf. also Ref. 56,

$$
\psi^{(l)}\left(\frac{2}{3}\right)=(-1)^{l+1} l !\left(3^{l+1}-1\right) \zeta_{l+1}-\psi^{(l)}\left(\frac{1}{3}\right) .
$$

For even values of $l, \psi^{(l)}(1 / 3)$ and $\psi^{(l)}(2 / 3)$ are linear in $\pi^{l+1} \sqrt{3}$ and $\zeta_{l+1}$, with

$$
\begin{aligned}
& \psi^{(2)}\left(\frac{1}{3}\right)=-\frac{4}{9} \sqrt{3} \pi^{3}-26 \zeta_{3}, \\
& \psi^{(2)}\left(\frac{2}{3}\right)=\frac{4}{9} \sqrt{3} \pi^{3}-26 \zeta_{3}, \\
& \psi^{(4)}\left(\frac{1}{3}\right)=-\frac{16}{3} \sqrt{3} \pi^{5}-2904 \zeta_{5}, \\
& \psi^{(4)}\left(\frac{2}{3}\right)=\frac{16}{3} \sqrt{3} \pi^{5}-2904 \zeta_{5}, \text { etc. }
\end{aligned}
$$

Therefore, only for odd values of $l$ one new basis element due to $\psi^{(2 l+1)}(1 / 3)$ contributes.

The two values at argument $1 / 4$ and $3 / 4$ are related, see, e.g., Ref. 57:

$$
\begin{aligned}
\psi^{(2 l-1)}\left(\frac{1}{4}\right) & =\frac{4^{2 l-1}}{2 l}\left[\pi^{2 l}\left(2^{2 l}-1\right)\left|B_{2 l}\right|+2(2 l) ! \beta_{D}(2 l)\right], \\
\psi^{(2 l-1)}\left(\frac{3}{4}\right) & =\frac{4^{2 l-1}}{2 l}\left[\pi^{2 l}\left(2^{2 l}-1\right)\left|B_{2 l}\right|-2(2 l) ! \beta_{D}(2 l)\right], \\
\psi^{(2 l)}\left(\frac{1}{4}\right) & =-2^{2 l-1}\left[\pi^{2 l+1}\left|E_{2 l}\right|+2(2 l) !\left(2^{2 l+1}-1\right) \zeta_{2 l+1}\right], \\
\psi^{(2 l)}\left(\frac{3}{4}\right) & =+2^{2 l-1}\left[\pi^{2 l+1}\left|E_{2 l}\right|-2(2 l) !\left(2^{2 l+1}-1\right) \zeta_{2 l+1}\right] .
\end{aligned}
$$

Here, $E_{2 l}$ denote the Euler numbers. ${ }^{58-61}$ They are generated by

$$
\frac{2}{\exp (x)+\exp (-x)}=\sum_{n=0}^{\infty} \frac{E_{n}}{n !} x^{n}, \quad E_{2 n+1}=0 \quad \text { for } n>1 .
$$

$\beta_{D}$ is the Dirichlet $\beta$-function ${ }^{52,62}$

$$
\beta_{D}(l)=\sum_{k=0}^{\infty} \frac{(-1)^{k}}{(2 k+1)^{l}}=\operatorname{Ti}_{l}(1), l \in \mathbb{N}_{+},
$$

which is also given by the inverse tangent integral $\mathrm{Ti}_{l}(x),{ }^{63,64}$ being related to Clausen integrals. ${ }^{65}$ The special value for $l=2$ yields Catalan's constant: ${ }^{44}$

$$
\sum_{k=0}^{\infty} \frac{(-1)^{k}}{(2 k+1)^{2}}=\mathbf{C}
$$

At even values of $l$ no new constants appear. Odd values contribute with $\operatorname{Ti}(2 l)$.

For $z=1 / 5,2 / 5,3 / 5,4 / 5$, the relations

$$
\psi^{(l)}\left(\frac{3}{5}\right)=(-1)^{l}\left[\psi^{(l)}\left(\frac{2}{5}\right)+\left.\pi \frac{d^{l}}{d z^{l}} \cot (\pi z)\right|_{z=2 / 5}\right],
$$




$$
\begin{gathered}
\psi^{(l)}\left(\frac{4}{5}\right)=(-1)^{l}\left[\psi^{(l)}\left(\frac{1}{5}\right)+\left.\pi \frac{d^{l}}{d z^{l}} \cot (\pi z)\right|_{z=1 / 5}\right], \\
l !(-1)^{l+1} \zeta_{l+1}\left[5^{l+1}-1\right]=\psi^{(l)}\left(\frac{1}{5}\right)+\psi^{(l)}\left(\frac{2}{5}\right)+\psi^{(l)}\left(\frac{3}{5}\right)+\psi^{(l)}\left(\frac{4}{5}\right)
\end{gathered}
$$

hold. For even values of $l, \psi^{(l)}(2 / 5)$ is thus dependent on $\psi^{(l)}(1 / 5)$.

For $z=1 / 6,5 / 6$, the relation

$$
\psi^{(l)}\left(\frac{5}{6}\right)=l !(-1)^{l+1} \zeta_{l+1}\left[6^{l+1}-3^{l+1}-2^{l+1}+1\right]-\psi^{(l)}\left(\frac{1}{6}\right)
$$

holds. The reflection formula (5.39) relates $\psi^{(l)}(1 / 6)$ and $\psi^{(l)}(5 / 6)$ for even values of $l$ to linear combinations of $\pi^{l+1} \sqrt{3}$ and $\zeta_{2 l+1}$. For odd $l$, the $\psi$-values can be expressed by $\psi^{(l)}(1 / 6)$. The shift-formula by $1 / 2$

$$
2^{l+1} \psi^{(l)}(2 z)=\psi^{(l)}(z)+\psi^{(l)}\left(z+\frac{1}{2}\right)
$$

implies

$$
\psi^{(l)}\left(\frac{1}{6}\right)=\left(2^{l+1}+1\right) \psi^{(l)}\left(\frac{1}{3}\right)+(-1)^{l} l !\left(3^{l+1}-1\right) \zeta_{l+1},
$$

and relates all values to $\psi^{(l)}(1 / 3)$.

For $z=1 / 8,3 / 8,5 / 8,7 / 8$, one obtains

$$
\psi^{(l)}\left(\frac{1}{8}\right)+\psi^{(l)}\left(\frac{3}{8}\right)+\psi^{(l)}\left(\frac{5}{8}\right)+\psi^{(l)}\left(\frac{7}{8}\right)=l !(-1)^{l+1} \zeta_{l+1} 4^{l+1}\left(2^{l+1}-1\right) .
$$

Due to (5.41) and (5.39) the corresponding $\psi$-values can be expressed by $\psi^{(l)}(1 / 8)$ :

$$
\begin{aligned}
& \psi^{(l)}\left(\frac{3}{8}\right)=2^{l+1} \psi^{(l)}\left(\frac{3}{4}\right)-(-1)^{l}\left[\psi^{(l)}\left(\frac{1}{8}\right)+\left.\pi \frac{d^{l}}{d z^{l}} \cot (\pi z)\right|_{z=1 / 8}\right] \\
& \psi^{(l)}\left(\frac{5}{8}\right)=2^{l+1} \psi^{(l)}\left(\frac{1}{4}\right)-\psi^{(l)}\left(\frac{1}{8}\right), \\
& \psi^{(l)}\left(\frac{7}{8}\right)=(-1)^{l}\left[\psi^{(l)}\left(\frac{1}{8}\right)+\left.\pi \frac{d^{l}}{d z^{l}} \cot (\pi z)\right|_{z=1 / 8}\right] .
\end{aligned}
$$

For $z=1 / 10,3 / 10,7 / 10,9 / 10$, two reflection relations and

$$
\psi^{(l)}\left(\frac{1}{10}\right)+\psi^{(l)}\left(\frac{3}{10}\right)+\psi^{(l)}\left(\frac{7}{10}\right)+\psi^{(l)}\left(\frac{9}{10}\right)=l !(-1)^{l+1} \zeta_{l+1}\left[10^{l+1}-5^{l+1}-2^{l+1}+1\right]
$$

hold. By the shift relation (5.59), one obtains

$$
\begin{aligned}
& \psi^{(l)}\left(\frac{1}{10}\right)=2^{l+1} \psi^{(l)}\left(\frac{1}{5}\right)-\psi^{(l)}\left(\frac{3}{5}\right), \\
& \psi^{(l)}\left(\frac{3}{10}\right)=2^{l+1} \psi^{(l)}\left(\frac{3}{5}\right)-\psi^{(l)}\left(\frac{4}{5}\right), \\
& \psi^{(l)}\left(\frac{7}{10}\right)=2^{l+1} \psi^{(l)}\left(\frac{2}{5}\right)-\psi^{(l)}\left(\frac{1}{5}\right),
\end{aligned}
$$




$$
\psi^{(l)}\left(\frac{9}{10}\right)=2^{l+1} \psi^{(l)}\left(\frac{4}{5}\right)-\psi^{(l)}\left(\frac{2}{5}\right) .
$$

No new constants contribute.

For $z=1 / 12,5 / 12,7 / 12,11 / 12$, two reflection relations and

$$
\begin{aligned}
\psi^{(l)}\left(\frac{1}{12}\right)+\psi^{(l)}\left(\frac{5}{12}\right)+\psi^{(l)}\left(\frac{7}{12}\right)+\psi^{(l)}\left(\frac{11}{12}\right)= & l !(-1)^{l+1} \zeta_{l+1} 2^{l+1} \\
& \times\left(6^{l+1}-3^{l+1}-2^{l+1}+1\right)
\end{aligned}
$$

hold. All values can be expressed by $\psi^{(l)}(1 / 12)$ :

$$
\begin{aligned}
& \psi^{(l)}\left(\frac{5}{12}\right)=(-1)^{l}\left[2^{l+1} \psi^{(l)}\left(\frac{1}{6}\right)-\psi^{(l)}\left(\frac{1}{12}\right)+\left.\pi \frac{d^{l}}{d z^{l}} \cot (\pi z)\right|_{z=7 / 12}\right], \\
& \psi^{(l)}\left(\frac{7}{12}\right)=2^{l+1} \psi^{(l)}\left(\frac{1}{6}\right)-\psi^{(l)}\left(\frac{1}{12}\right), \\
& \psi^{(l)}\left(\frac{11}{12}\right)=(-1)^{l}\left[\psi^{(l)}\left(\frac{1}{12}\right)+\left.\pi \frac{d^{l}}{d z^{l}} \cot (\pi z)\right|_{z=1 / 12}\right], \text { etc. }
\end{aligned}
$$

Equation (5.41) yields

$$
\psi^{(l)}\left(\frac{5}{12}\right)=3^{l+1} \psi^{(l)}\left(\frac{1}{4}\right)-\psi^{(l)}\left(\frac{3}{4}\right)-\psi^{(l)}\left(\frac{1}{12}\right) .
$$

For odd values of $l$, one obtains

$$
\psi^{(l)}\left(\frac{1}{12}\right)=2^{l} \psi^{(l)}\left(\frac{1}{6}\right)+\frac{1}{2}\left[3^{l+1} \psi^{(l)}\left(\frac{1}{4}\right)-\psi^{(l)}\left(\frac{3}{4}\right)+\left.\pi \frac{d^{l}}{d z^{l}} \cot (\pi z)\right|_{z=7 / 12}\right] .
$$

Equation (5.70) does not imply a further relation. $\psi^{(l)}(1 / 12)$ contributes as a new constant for even values of $l$.

For even values of $l=2 q$ the Hurwitz $\zeta$-function (5.5) obeys the representation ${ }^{66}$

$$
\begin{aligned}
\sum_{k=0}^{\infty} \frac{1}{(2 q k+2 p-1)^{n}} & =\frac{1}{(2 q)^{n}} \zeta_{\mathrm{H}}\left(n, \frac{2 p-1}{2 q}\right) \\
& =\frac{1}{q} \sum_{k=1}^{q}\left[C_{n}\left(\frac{k}{q}\right) \cos \left(\frac{(2 p-1) k \pi}{q}\right)+S_{n}\left(\frac{k}{q}\right) \sin \left(\frac{(2 p-1) k \pi}{q}\right)\right],
\end{aligned}
$$

where $C_{v}$ and $S_{v}$ are represented by the Legendre $\chi$-function ${ }^{67}$ (5.77),

$$
\chi_{v}(z)=\frac{1}{2}\left[\operatorname{Li}_{v}(z)-\mathrm{Li}_{v}(-z)\right]
$$

with $^{92}$

$$
\begin{aligned}
& C_{v}(x)=\operatorname{Re} \chi_{v}(\exp (i \pi x)) \\
& S_{v}(x)=\operatorname{Im} \chi_{v}(\exp (i \pi x))
\end{aligned}
$$


For $v \in \mathbb{N},(5.78)$ can be represented by Euler polynomials ${ }^{68}$ and powers of $\pi$,

$$
\begin{aligned}
C_{2 n}\left(\frac{p}{q}\right) & =\frac{(-1)^{n}}{4(2 n-1) !} \pi^{2 n} E_{2 n-1}\left(\frac{p}{q}\right), \\
S_{2 n+1}\left(\frac{p}{q}\right) & =\frac{(-1)^{n}}{4(2 n) !} \pi^{2 n+1} E_{2 n}\left(\frac{p}{q}\right), p, q \in \mathbb{N}_{+}, p \leq q
\end{aligned}
$$

with

$$
\frac{2 \exp (x t)}{\exp (t)+1}=\sum_{n=0}^{\infty} E_{n}(x) \frac{t^{n}}{n !}
$$

Since

$$
\psi^{(m-1)}\left(\frac{1}{l}\right)=(-1)^{m} l^{m}(m-1) ! \sum_{k=0}^{\infty} \frac{1}{(l k+1)^{m}},
$$

in particular, $\psi^{(l)}(1 / 8)$ and $\psi^{(l)}(1 / 12), l \in \mathbb{N}_{+}, l \geq 1$ consist of one term containing an integer power of $\pi$ and a second term $\propto \operatorname{Re}(\operatorname{Im})\left(\chi_{l+1}(r)\right), r \in \mathbb{Q}$ according to (5.76) and (5.78).

In conclusion, the representation of the single sums of weight $\mathbf{w} \geq 2$ and $l \leq 6$ requires the additional constants

$$
\zeta_{2 k+1}, \psi^{(2 k+1)}\left(\frac{1}{3}\right), \mathrm{Ti}_{2 k}(1), \psi^{(k)}\left(\frac{1}{5}\right), \psi^{(2 k+1)}\left(\frac{2}{5}\right), \psi^{(k)}\left(\frac{1}{8}\right), \psi^{(2 k)}\left(\frac{1}{12}\right), k \in \mathbb{N}_{+} .
$$

Finally, we would like to comment on a relation in Ramanujan's notebooks ${ }^{69}$ Chap. 9, (11.3), which was claimed to involve a cyclotomic harmonic sum,

$$
\begin{aligned}
& G(1)=\frac{1}{8} \sum_{r=1}^{\infty} \frac{1}{r^{3}} \sum_{s=1}^{r} \frac{1}{2 l-1} \\
& H(1)=\frac{\pi}{4} \sum_{r=0}^{\infty} \frac{(-1)^{r}}{(4 r+1)^{3}}-\frac{\pi}{3 \sqrt{3}} \sum_{r=0}^{\infty} \frac{1}{(2 r+1)^{3}}
\end{aligned}
$$

with

$$
G(1)=H(1) .
$$

This relation is unfortunately incorrect, cf. Ref. 69 , p. 257. G(1) may be given by the following representation in multiple zeta values introducing nested harmonic sums, ${ }^{70}$

$$
\begin{aligned}
G(1) & =\frac{7}{16} \sigma_{3,1}+\frac{1}{2} \sigma_{-3,1}=-\frac{53}{160} \zeta_{2}^{2}-\frac{1}{4} \zeta_{2} \ln ^{2}(2)+\frac{7}{8} \zeta_{3} \ln (2)+\frac{1}{24} \ln ^{4}(2)+\mathrm{Li}_{4}\left(\frac{1}{2}\right) \\
& \simeq 0.16227193947148339072
\end{aligned}
$$

using the representations in Ref. 11. At present, no relation between the special constants used in (5.87) is known. On the other hand, $H(1)$ is given by

$$
\begin{aligned}
H(1) & =-\frac{\pi}{2048}\left\{8\left[\pi^{3}+28\left(1+\frac{8}{9} \sqrt{3}\right) \zeta_{3}\right]+\psi^{(2)}\left(\frac{1}{8}\right)\right\} \\
& \simeq 0.14402290986880995023 .
\end{aligned}
$$


TABLE III. Basis representations of the infinite cyclotomic harmonic sums over the alphabet $\left\{( \pm 1)^{k} / k,( \pm 1)^{k} /(2 k+1)\right\}$ after applying the stuffle $(A)$, shuffle (SH) relations, their combination, and their application together with the three multiple argument relations $\left(H_{1}, H_{2}, M\right)$, as far these lead not to new quantities. In the latter case we quote the cumulative number of basis elements appearing at the new weight.

\begin{tabular}{lrrrcccc}
\hline \hline Weight & \multicolumn{1}{c}{${ }^{\prime} N_{S}$} & \multicolumn{1}{c}{$A$} & $\mathrm{SH}$ & $A+\mathrm{SH}$ & $A+\mathrm{SH}+H_{1}$ & $A+\mathrm{SH}+H_{1}+H_{2}$ & $A+\mathrm{SH}+H_{1}+H_{2}+M$ \\
\hline 1 & 4 & 4 & 4 & 4 & 4 & 3 & 3 \\
2 & 20 & 10 & 13 & 3 & 3 & 2 & 1 \\
3 & 100 & 40 & 46 & 6 & 6 & 5 & 3 \\
4 & 500 & 150 & 163 & 10 & 10 & 9 & 6 \\
5 & 2500 & 624 & 650 & 21 & 21 & 19 & 25 \\
6 & 12500 & 2580 & 2635 & 36 & 36 & 34 & 25 \\
\hline \hline
\end{tabular}

\section{B. Infinite multiple sums at higher depth}

As a further extension of the infinite cyclotomic harmonic sums ${ }^{11}$ we consider the iterated summation of the terms (4.60). The corresponding sums diverge if the first indices have the pattern $c_{i}=1, s_{i}=1,(2.1)$. However, these divergences can be regulated by polynomials in $\sigma_{0}$ and cyclotomic harmonic sums, which are convergent for $N \rightarrow \infty$, very similar to the case of the usual harmonic sums. ${ }^{7,8}$ We study the relations given in Sec. IV C supplemented by those of the shuffle algebra $(\mathrm{SH})$ of the cyclotomic harmonic polylogarithms (at argument $x=1$ ), Eq. (3.21). In Table III we present the number of basis elements obtained applying the respective relations up to weight $w=6$. The representation of all sums was computed by means of computer algebra in an explicit form. We derive the cumulative basis, quoting only the new elements in the next weight. Up to $w=4$ we derive also suitable integral representations over known functions. One possible choice of basis elements is

$w=1:$

$$
\begin{aligned}
\sigma_{\{1,0,1\}} & =\sigma_{0}, \\
\sigma_{\{1,0,-1\}} & =-\ln (2), \\
\sigma_{\{2,1,-1\}} & =-1+\frac{\pi}{4} .
\end{aligned}
$$

$w=2:$

$$
\sigma_{\{2,1,-2\}}=-1+\mathbf{C} \text {. }
$$

w = 3:

$$
\begin{aligned}
\sigma_{\{1,0,3\}} & =\zeta_{3}, \\
\sigma_{\{1,0,-2\},\{2,1,-1\}} & =\frac{\pi^{2}}{12}-\frac{\pi^{3}}{48}+\frac{1}{2} \int_{0}^{1} d x \frac{\sqrt{x}}{x+1} \operatorname{Li}_{2}(x), \\
\sigma_{\{2,1,-2\},\{1,0,-1\}} & =-\mathbf{C} \ln (2)+\int_{0}^{1} d x \frac{1}{1+x} \frac{\chi_{2}(\sqrt{x})}{\sqrt{x}} .
\end{aligned}
$$

$w=4$ :

$$
\begin{aligned}
\sigma_{\{1,0,-1\},\{1,0,1\},\{1,0,1\},\{1,0,1\}} & =-\operatorname{Li}_{4}\left(\frac{1}{2}\right), \\
\sigma_{\{2,1,-4\}} & =-1-i \chi_{4}(i), \\
\sigma_{\{2,1,-3\},\{2,1,-1\}} & =i\left(1-\frac{\pi}{4}\right) \chi_{3}(i)+\frac{1}{2} \int_{0}^{1} d x \frac{1}{x+1} \chi_{3}(\sqrt{x}),
\end{aligned}
$$




$$
\begin{aligned}
\sigma_{\{1,0,-2\},\{1,0,-1\},\{2,1,-1\}=} & \left(\frac{1}{4} \pi^{2} \ln (2)-\frac{5}{8} \zeta_{3}\right)\left(1-\frac{\pi}{4}\right) \\
& +\frac{1}{2} \int_{0}^{1} \frac{\sqrt{x}}{1+x}\left[\left(\operatorname{Li}_{2}(-x)+\frac{\pi^{2}}{12}\right) \ln (1-x)\right. \\
& \left.+\frac{1}{2} \mathrm{~S}_{1,2}\left(x^{2}\right)-\mathrm{S}_{1,2}(x)-\mathrm{S}_{1,2}(-x)\right], \\
\sigma_{\{1,0,-2\},\{2,1,-1\},\{1,0,1\}=} & -\frac{1}{2} \int_{0}^{1} d x \frac{\sqrt{x}}{1+x}[\ln (1-\sqrt{x})-\ln (1+\sqrt{x})] \operatorname{Li}_{2}(x) \\
& -\ln (2) \int_{0}^{1} d x \frac{\sqrt{x}}{1+x} \operatorname{Li}_{2}(x)+\frac{\pi^{2}}{24}[\ln (2) \pi-2 \mathbf{C}], \\
\sigma_{\{1,0,-2\},\{2,1,-1\},\{2,1,1\}=} & -\frac{1}{4} \int_{0}^{1} d x \frac{\sqrt{x}}{1+x} \ln (1-x) \operatorname{Li}_{2}(x) \\
& -\frac{1}{2}[1-\ln (2)] \int_{0}^{1} d x \frac{\sqrt{x}}{1+x} \operatorname{Li}_{2}(x) \\
& +\frac{\pi^{2}}{24}\left[\frac{\pi}{2}\left[1-\frac{1}{2} \ln (2)\right]-\mathbf{C}\right] .
\end{aligned}
$$

Here, $S_{1,2}(x)$ denotes a Nielsen integral: ${ }^{71}$

$$
S_{1,2}(x)=\frac{1}{2} \int_{0}^{x} \frac{d z}{z} \ln ^{2}(1-z) .
$$

At weight $\mathrm{w}=5,6$ we give only a few integral representations. They can, in general, be obtained form the Mellin transforms setting the kernel $x^{N} \rightarrow 1$. The following basis elements are obtained: $w=5$ :

$$
\sigma_{\{1,0,5\}}=\zeta_{5},
$$

$$
\begin{aligned}
& \sigma_{\{1,0,-1\},\{1,0,1\},\{1,0,1\},\{1,0,1\},\{1,0,1\}}=-\operatorname{Li}_{5}\left(\frac{1}{2}\right) \\
& \sigma_{\{1,0,-4\},\{2,1,-1\}}=\frac{7}{720} \pi^{4}-\frac{7}{2880} \pi^{5}+\frac{1}{2} \int_{0}^{1} d x \frac{\sqrt{x}}{1+x} \operatorname{Li}_{4}(x), \\
& \sigma_{\{1,0,4\},\{2,1,-1\}}=-\frac{\pi^{4}}{90}+\frac{\pi^{5}}{360}+\frac{1}{2} \int_{0}^{1} d x \frac{\sqrt{x}}{1+x} \operatorname{Li}_{4}(-x),
\end{aligned}
$$

$$
\sigma_{\{2,1,-4\},\{1,0,-1\}}, \sigma_{\{1,0,-3\},\{1,0,-1\},\{2,1,1\}}, \sigma_{\{1,0,-3\},\{2,1,-1\},\{2,1,-1\}}, \sigma_{\{1,0,3\},\{2,1,-1\},\{2,1,-1\}},
$$$$
\sigma_{\{2,1,-3\},\{2,1,-1\},\{2,1,1\}}, \sigma_{\{1,0,-2\},\{1,0,-1\},\{1,0,-1\},\{2,1,-1\}}, \sigma_{\{1,0,-2\},\{1,0,-1\},\{2,1,-1\},\{1,0,-1\}},
$$$$
\sigma_{\{1,0,-2\},\{2,1,-1\},\{1,0,1\},\{1,0,1\}}, \sigma_{\{1,0,-2\},\{2,1,-1\},\{2,1,1\},\{1,0,1\}}
$$

$w=6$ :

$$
\sigma_{\{1,0,-5\},\{1,0,-1\}}=\sigma_{-5,-1},
$$




$$
\begin{aligned}
& \sigma_{\{1,0,-1\},\{1,0,1\},\{1,0,1\},\{1,0,1\},\{1,0,1\},\{1,0,1\}}=-\operatorname{Li}_{6}\left(\frac{1}{2}\right), \\
& \sigma_{\{2,1,-6\}}=-1-i \chi_{6}(i) \\
& \sigma_{\{1,0,-2\},\{2,1,-1\},\{2,1,1\},\{1,0,1\},\{1,0,1\}}, \sigma_{\{1,0,-2\},\{2,1,-1\},\{1,0,1\},\{1,0,1\},\{1,0,1\}}, \\
& \sigma_{\{1,0,-2\},\{1,0,-1\},\{1,0,-1\},\{2,1,-1\},\{2,1,1\}}, \sigma_{\{1,0,-2\},\{1,0,-1\},\{1,0,-1\},\{2,1,-1\},\{1,0,1\}}, \\
& \sigma_{\{1,0,-2\},\{1,0,-1\},\{1,0,-1\},\{1,0,-1\},\{2,1,-1\}}, \sigma_{\{1,0,3\},\{2,1,-1\},\{2,1,-1\},\{1,0,1\}}, \\
& \sigma_{\{1,0,-3\},\{1,0,-1\},\{2,1,1\},\{1,0,1\}}, \sigma_{\{1,0,-3\},\{1,0,-1\},\{1,0,1\},\{2,1,1\}}, \sigma_{\{2,1,-3\},\{2,1,-1\},\{2,1,-1\},\{2,1,-1\}}, \\
& \sigma_{\{1,0,-3\},\{2,1,-1\},\{2,1,-1\},\{1,0,-1\}}, \sigma_{\{1,0,-3\},\{2,1,-1\},\{1,0,-1\},\{2,1,-1\}}, \sigma_{\{1,0,-3\},\{1,0,-1\},\{2,1,-1\},\{2,1,-1\}}, \\
& \sigma_{\{2,1,4\},\{1,0,-1\},\{2,1,-1\}}, \sigma_{\{1,0,4\},\{2,1,-1\},\{1,0,-1\}}, \sigma_{\{1,0,4\},\{1,0,-1\},\{2,1,-1\}}, \\
& \sigma_{\{2,1,-4\},\{1,0,-1\},\{2,1,1\}}, \sigma_{\{2,1,-4\},\{1,0,-1\},\{1,0,1\}}, \sigma_{\{1,0,-4\},\{2,1,-1\},\{2,1,1\}}, \\
& \sigma_{\{1,0,-4\},\{2,1,-1\},\{1,0,1\}}, \sigma_{\{1,0,-4\},\{1,0,-1\},\{2,1,-1\}}, \sigma_{\{2,1,-4\},\{2,1,-2\}}, \\
& \sigma_{\{2,1,-5\},\{2,1,-1\}} \text {. }
\end{aligned}
$$

Recently, infinite sums of a type proposed in Refs. 72, 73, and 93 were studied in Ref. 75, Eqs. $(11 \mathrm{a}-\mathrm{c})$. They can be expressed in terms of sums studied in this section:

$$
\begin{gathered}
\mathcal{J}_{1}(r)=\frac{1}{2} \sum_{k=0}^{\infty} \frac{[\psi(k+1 / 2)-\psi(1 / 2)]}{(2 k+1)^{r}}=\sigma_{\{2,1, r\}}-\sigma_{\{2,1, r+1\}}+\sigma_{\{2,1, r+1\},\{2,1,1\}}, \\
\mathcal{J}_{2}(r)=\frac{1}{2} \sum_{k=0}^{\infty} \frac{[\psi(k+1 / 2)-\psi(1 / 2)]}{(2 k)^{r}}=\frac{1}{2^{r}} \sigma_{\{1,0, r\},\{2,-1,1\},}, \\
\mathcal{M}(r)=\frac{1}{2} \sum_{k=0}^{\infty} \frac{[\psi(k+1)+\gamma]}{(2 k+1)^{r}}=\frac{1}{2} \sigma_{\{2,1, r\},\{1,0,1\} .}
\end{gathered}
$$

Moreover, values of usual harmonic polylogarithms ${ }^{26}$ at $x=1, \int_{0}^{1} d t\left[\operatorname{Li}_{p}( \pm t)-\mathrm{Li}_{p}( \pm 1)\right] /(1 \pm t)$, are discussed. In part, these integrals refer to all three letters of the corresponding alphabet. The corresponding representations involve infinite harmonic sums of depth $d>1$ naturally, as, e.g., for

$$
\int_{0}^{1} d x \frac{\operatorname{Li}_{5}(x)}{1+x}=H_{-1,0,0,0,1}(1)=-\frac{15}{16} \zeta_{5} \ln (2)+\sigma_{-5,-1}
$$

and in similar cases of higher weight, see Refs. 8, 11, and 26.

\section{Infinite multiple sums with more cyclotomic letters}

Let us now consider more cyclotomic letters. We study the sums up to weight $\mathbf{w}=2$ and cyclotomy $I=20,,^{94}$ based on the sets of the non-alternating and alternating sums using the letters

$$
\frac{( \pm 1)^{k}}{(l k+m)^{n}}, \quad 1 \leq n \leq 2,1 \leq l \leq 20, m<l .
$$

We use the stuffle (quasi-shuffle) relations for the sums, the shuffle relations on the side of the associated cyclotomic harmonic polylogarithms, and the multiple argument relations for these sums, cf. Sec. IV C. In some cases the latter request to include sums which are outside the above pattern. In this case the corresponding relations are not accounted for. At $w=1$ the respective numbers of basis elements is summarized in Table IV. 
TABLE IV. The number of the $\mathbf{w}=1$ cyclotomic harmonic sums (5.116) up to $I=20$, the basis elements at fixed value of $\mathrm{I}$, and the new basis elements in ascending sequence.

\begin{tabular}{lrrrrrrrrrrrrrrrrrrrrrrrrr}
\hline \hline \multicolumn{1}{c}{1} & 1 & 2 & 3 & 4 & 5 & 6 & 7 & 8 & 9 & 10 & 11 & 12 & 13 & 14 & 15 & 16 & 17 & 18 & 19 & 20 \\
\hline Sums & 2 & 4 & 6 & 8 & 10 & 12 & 14 & 16 & 18 & 20 & 22 & 24 & 26 & 28 & 30 & 32 & 34 & 36 & 38 & 40 \\
Basis & 2 & 3 & 4 & 5 & 6 & 6 & 8 & 9 & 8 & 10 & 12 & 10 & 14 & 14 & 11 & 17 & 18 & 14 & 20 & 18 \\
New basis & 2 & 1 & 2 & 2 & 4 & 1 & 6 & 4 & 4 & 3 & 10 & 2 & 12 & 5 & 3 & 8 & 16 & 4 & 18 & 6 \\
sums & & & & & & & & & & & & & & & & & & & & \\
\hline \hline
\end{tabular}

The reflection relation (5.39) for the $\psi$-functions for $x \leftrightarrow(1-x)$ implies that there are at most $l+1$ basis elements. We showed that the use of the above analytic representations and the shuffle, stuffle, and multiple argument relations lead to the same number of basis elements for $l \leq 6$ in the non-alternating and alternating case.

The independent sums at $\mathrm{W}=1$ up to $\mathrm{I}=6$ are

$$
\begin{aligned}
& \sigma_{\{1,0,1\}}, \sigma_{\{1,0,-1\}}, \sigma_{\{2,1,-1\}}, \sigma_{\{3,1,1\}}, \sigma_{\{3,1,-1\}}, \sigma_{\{4,1,-1\}}, \sigma_{\{4,3,-1\}}, \sigma_{\{5,1,1\}}, \sigma_{\{5,1,-1\}}, \\
& \sigma_{\{5,2,-1\}}, \sigma_{\{5,3,-1\}}, \sigma_{\{6,1,-1\}},
\end{aligned}
$$

see Secs. V A 1 and V A 2 for equivalent representations.

The dependent sums up to $I=6$ are

$$
\begin{aligned}
\sigma_{\{2,1,1\}} & =-1-\sigma_{\{1,0,-1\}}+\frac{1}{2} \sigma_{\{1,0,1\}}, \\
\sigma_{\{3,2,1\}} & =-\frac{1}{2}-\frac{1}{3} \sigma_{\{1,0,-1\}}-\sigma_{\{3,1,-1\}}+\sigma_{\{3,1,1\}}, \\
\sigma_{\{3,2,-1\}} & =\frac{1}{2}+\frac{2}{3} \sigma_{\{1,0,-1\}}+\sigma_{\{3,1,-1\}}, \\
\sigma_{\{4,1,1\}} & =-\frac{1}{2}-\frac{3}{4} \sigma_{\{1,0,-1\}}+\frac{1}{4} \sigma_{\{1,0,1\}}+\frac{1}{2} \sigma_{\{2,1,-1\}}, \\
\sigma_{\{4,3,1\}} & =-\frac{5}{6}-\frac{3}{4} \sigma_{\{1,0,-1\}}+\frac{1}{4} \sigma_{\{1,0,1\}}-\frac{1}{2} \sigma_{\{2,1,-1\}}, \\
\sigma_{\{5,4,-1\}} & =\frac{7}{12}+\frac{4}{5} \sigma_{\{1,0,-1\}}+\sigma_{\{5,1,-1\}}-\sigma_{\{5,2,-1\}}+\sigma_{\{5,3,-1\}}, \\
\sigma_{\{5,2,1\}} & =\frac{1}{5} \sigma_{\{1,0,-1\}}+\sigma_{\{5,1,1\}}-\sigma_{\{5,2,-1\}}, \\
\sigma_{\{5,3,1\}} & =-\frac{1}{3}-\frac{1}{5} \sigma_{\{1,0,-1\}}-\sigma_{\{5,1,-1\}}+\sigma_{\{5,1,1\}}, \\
& =-\frac{1}{5} \sigma_{\{1,0,-1\}}-\sigma_{\{5,1,-1\}}+\sigma_{\{5,1,1\}}-\sigma_{\{5,3,-1\}},
\end{aligned}
$$




$$
\begin{aligned}
\sigma_{\{6,5,1\}} & \left.=-\frac{7}{10}-\frac{2}{3} \sigma_{\{1,0,-1}\right\}-\sigma_{\{3,1,-1\}}+\frac{1}{2} \sigma_{\{3,1,1\}}, \\
\sigma_{\{6,5,-1\}} & =\frac{2}{15}+\frac{4}{3} \sigma_{\{2,1,-1\}}-\sigma_{\{6,1,-1\}}, \quad \text { etc. }
\end{aligned}
$$

The remaining sums are related to those given in (5.117) to (5.129). The following counting relations for the basis elements were tested up to $\mathrm{I}=700$ using computer algebra methods. Let $p, p_{i}, q$ be pairwise distinct primes $>2$, and let $k, k_{i}$ be positive integers. The number of basis elements at $\mathrm{w}=1$ and cyclotomy I are given by

$$
\varphi(l)=\left\{\begin{array}{ll}
l+1, & l=1 \text { or } l=2^{k} \\
(p-1) p^{k-1}+2, & l=p^{k} \\
2 \varphi\left(2^{k-1} \prod_{i=1}^{n} p_{i}^{k_{i}}\right)-n-1, & l=2^{k} \prod_{i=1}^{n} p_{i}^{k_{i}} \\
(q-1) \varphi\left(\prod_{i=1}^{n} p_{i}^{k_{i}}\right)-n(q-2)-q+3, & l=q \prod_{i=1}^{n} p_{i}^{k_{i}} \\
q \varphi\left(q^{k-1} \prod_{i=1}^{n} p_{i}^{k_{i}}\right)-(n+2)(q-1), & l=q^{k} \prod_{i=1}^{n} p_{i}^{k_{i}}, k>1
\end{array} .\right.
$$

Let us now consider the case $\mathbf{w}=2$. Applying the relations given in Sec. IV C and the shuffle algebra of the cyclotomic harmonic polylogarithms at argument $x=1$, the results given in Table V are obtained for the number of basis elements. Again, we solved the corresponding linear systems using computer algebra methods and derived the representations for the dependent sums analytically.

The number of the weight $\mathrm{w}=2$ infinite sums for cyclotomy $\mathrm{I}$ is

$$
N_{S}=2 l(2 l+1) \text {. }
$$

\begin{tabular}{|c|c|c|c|c|c|c|c|}
\hline 1 & $N_{S}$ & $\mathrm{SH}$ & A & $A+\mathrm{SH}$ & $A+\mathrm{SH}+H_{1}$ & $A+\mathrm{SH}+H_{1}+H_{2}$ & $A+\mathrm{SH}+H_{1}+H_{2}+M$ \\
\hline 1 & 6 & 4 & 3 & 1 & 1 & 1 & 1 \\
\hline 2 & 20 & 13 & 10 & 3 & 3 & 2 & 1 \\
\hline 3 & 42 & 27 & 21 & 7 & 6 & 6 & 5 \\
\hline 4 & 72 & 46 & 36 & 12 & 11 & 10 & 3 \\
\hline 5 & 110 & 70 & 55 & 19 & 17 & 17 & 16 \\
\hline 6 & 156 & 99 & 78 & 27 & 25 & 24 & 5 \\
\hline 7 & 210 & 133 & 105 & 37 & 34 & 34 & 33 \\
\hline 8 & 272 & 172 & 136 & 48 & 45 & 44 & 12 \\
\hline 9 & 342 & 216 & 171 & 61 & 57 & 57 & 52 \\
\hline 10 & 420 & 265 & 210 & 75 & 71 & 70 & 22 \\
\hline 11 & 506 & 319 & 253 & 91 & 86 & 86 & 85 \\
\hline 12 & 600 & 378 & 300 & 108 & 103 & 102 & 21 \\
\hline 13 & 702 & 442 & 351 & 127 & 121 & 121 & 120 \\
\hline 14 & 812 & 551 & 406 & 147 & 141 & 140 & 49 \\
\hline 15 & 930 & 585 & 465 & 169 & 162 & 162 & 145 \\
\hline 16 & 1056 & 664 & 528 & 192 & 185 & 184 & 50 \\
\hline 17 & 1190 & 748 & 595 & 217 & 209 & 209 & 208 \\
\hline 18 & 1332 & 837 & 666 & 243 & 235 & 234 & 63 \\
\hline 19 & 1482 & 931 & 741 & 271 & 262 & 262 & 261 \\
\hline 20 & 1640 & 1030 & 820 & 300 & 291 & 290 & 74 \\
\hline
\end{tabular}

TABLE V. Number of basis elements of the $\mathbf{w}=2$ cyclotomic harmonic sums (5.116) up to cyclotomy I $=20$ after applying the quasi-shuffle algebra of the sums $(A)$, the shuffle algebra of the cyclotomic harmonic polylogarithms $(\mathrm{SH})$, and the three multiple argument relations $\left(H_{1}, H_{2}, M\right)$ of the sums. 
One may guess, based on the results for $I \leq 20$, counting relations for the length of the bases listed in Table V. We found for all but the last column:

$$
\begin{aligned}
N_{A}(l) & =l(2 l+1), \\
N_{\mathrm{SH}}(l) & =\frac{(5 l+3) l}{2}, \\
N_{A, \mathrm{SH}}(l) & =\frac{6 l^{2}+1-(-1)^{l}}{8}, \\
N_{A, \mathrm{SH}, H_{1}}(l) & =\frac{6 l^{2}-4 l+7-(-1)^{l}}{8}, \\
N_{A, \mathrm{SH}, H_{1}, H_{2}}(l) & =\frac{6 l^{2}-4 l+3\left(1-(-1)^{l}\right)}{8} .
\end{aligned}
$$

The latter relation (5.136) has been derived prior to us by Broadhurst: ${ }^{95}$

$$
N_{A, \mathrm{SH}, H_{1}, H_{2}}(l)=\frac{3}{4} l^{2}-\frac{1}{2} l+\mathrm{if}(\operatorname{modp}(1,2)=0,1,3 / 4),
$$

and the corresponding generating function

$$
f(x)=\left[1+\frac{x^{3}}{1+x}\right] \frac{1}{(1-x)^{3}}=\sum_{l=0}^{\infty} a(l) x^{l} .
$$

We conjecture that in case of $N_{A, \mathrm{SH}, H_{1}, H_{2}}(l)$ the $(M)$-relations lead to a reduction of one in the basis for I being a prime. Otherwise, quite significant reductions are obtained for which we do not know an explicit counting relation. The corresponding sequence is also not recorded yet in the data base. ${ }^{78}$

\section{GENERALIZED HARMONIC SUMS AT ROOTS OF UNITY}

In Sec. V we considered real representations for the infinite cyclotomic harmonic sums. These are related to the infinite generalized harmonic sums at the roots of unity. We define

$$
\lim _{N \rightarrow \infty} S_{k_{1}, \ldots, k_{m}}\left(x_{1}, \ldots, x_{m} ; N\right)=\sigma_{k_{1}, \ldots, k_{m}}\left(x_{1}, \ldots, x_{m}\right)
$$

with $S_{k_{1}, \ldots, k_{m}}\left(x_{1}, \ldots, x_{m} ; N\right)$ being a generalized harmonic sum (1.3), see also Refs. 13 and 14, and $x_{j} \in \mathcal{C}_{n}, n \geq 1$, with $\mathcal{C}_{n} \in\left\{e_{n} \mid e_{n}^{n}=1, e_{n} \in \mathbb{C}\right\} ; k_{1} \neq 1$ for $x_{1}=1$.

We seek the relations between the sums of $w=1,2$. They can be expressed in terms of polylogarithms by

$$
\begin{aligned}
\sigma_{\mathrm{w}}(x) & =\mathrm{Li}_{\mathrm{w}}(x), \quad \mathrm{w} \in \mathbb{N}, \mathrm{w} \geq 1, \\
\sigma_{1}(x) & =\mathrm{Li}_{1}(x)=-\ln (1-x), \\
\sigma_{1,1}(x, y) & =\mathrm{Li}_{2}(x)+\frac{1}{2} \ln ^{2}(1-x)+\mathrm{Li}_{2}\left(-\frac{x(1-y)}{1-x}\right), \\
\sigma_{1,1}\left(x, x^{*}\right) & =\mathrm{Li}_{2}(x)+\frac{1}{2} \ln ^{2}(1-x), \\
\mathrm{Li}_{\mathrm{w}}(x) & =\mathrm{Li}_{\mathrm{w}}^{*}\left(x^{*}\right),
\end{aligned}
$$


and ${ }^{*}$ denotes complex conjugation. Furthermore, the symmetric combination $\sigma_{1,1}(x, y)+\sigma_{1,1}(y, x)$ is given by ${ }^{13,14}$

$$
\sigma_{1,1}(x, y)+\sigma_{1,1}(y, x)=\ln (1-x) \ln (1-y)+\operatorname{Li}_{2}(x y) .
$$

Knowing the representations at $\mathbf{w}=1$ and the dilogarithms at the corresponding roots of unity one term $\sigma_{1,1}(y, x)$ may be expressed by (6.7).

In analyzing the functions $\sigma_{k_{1}, \ldots, k_{m}}\left(x_{1}, \ldots, x_{m}\right)$ with $x_{k}^{l}=1$, one may use the real representations of nested cyclotomic sums for $N \rightarrow \infty$, accounting for the respective sub-cycles at $d, d \mid l$, and complex conjugation.

An example is given by

$$
\left\{\left.e_{12}^{k}\right|_{k=1} ^{12}\right\} \equiv\left\{e_{12}, e_{6}, e_{4}, e_{3}, e_{12}^{5}, e_{2}, e_{12}^{5}, e_{3}^{*}, e_{4}^{*}, e_{6}^{*}, e_{12}^{*}, e_{1}\right\} .
$$

Here, we labeled the elements occurring in sub-cycles accordingly.

The polylogarithms $\operatorname{Li}_{1}\left(e_{l}^{k}\right)$ and $\operatorname{Li}_{2}\left(e_{l}^{k}\right)$ obey

$$
\begin{gathered}
\operatorname{Im}\left[\operatorname{Li}_{1}\left(e_{l}^{k}\right)\right]=\frac{l-2 k}{2 l} \pi, \\
\operatorname{Re}\left[\operatorname{Li}_{2}\left(e_{l}^{k}\right)\right]=\frac{6 k(k-l)+l^{2}}{6 l^{2}} \pi^{2} .
\end{gathered}
$$

More generally, PSLQ (Ref. 79 tests let to conjecture that $\operatorname{Im}\left[\operatorname{Li}_{n}\left(e_{l}^{k}\right)\right]=r_{n, l, k} \pi^{n}$ for $n$ odd and $\operatorname{Re}\left[\operatorname{Li}_{n}\left(e_{l}^{k}\right)\right]=r_{n, l, k} \pi^{n}$ for $n$ odd with $r_{n, l, k} \in \mathbb{Q}$.

Now we extend Proposition 2.3 of Ref. 19, where we consider generalized harmonic sums $S_{k_{1}, \ldots, k_{m}}\left(x_{1}, \ldots, x_{m} ; N\right)$ with $N \in \mathbb{N}, k_{i} \in \mathbb{N}_{+}, x_{i} \in \mathbb{C},\left|x_{i}\right| \leq 1$. Let $l \in \mathbb{N}_{+}$and

$$
y_{i}^{l}=x_{i}
$$

then

$$
S_{k_{1}, \ldots, k_{m}}\left(x_{1}, \ldots, x_{m} ; N\right)=\prod_{j=1}^{m} l^{k_{j}-1} \sum_{y_{i}^{l}=x_{i}} S_{k_{1}, \ldots, k_{m}}\left(y_{1}, \ldots, y_{m} ; l N\right) .
$$

Here, the sum is over the $l$ th roots of $x_{i}$ for $i \in[1, m]$. Equation (6.12) is called distribution relation. It follows from the Vieta's theorem ${ }^{80}$ for (6.11) and properties of symmetric polynomials. ${ }^{81}$ Equation (6.12) contains the well-known duplication relation, cf. Eq. (2.15). ${ }^{11}$

If also $x_{1} \neq 1$ for $k_{1}=1$, the limit

$$
\sigma_{k_{1}, \ldots, k_{m}}\left(x_{1}, \ldots, x_{m}\right)=\lim _{N \rightarrow \infty} S_{k_{1}, \ldots, k_{m}}\left(x_{1}, \ldots, x_{m} ; N\right)
$$

exists. One may apply (6.12) and (6.13) to roots of unity $x_{i}$ and $y_{j}$, i.e., $x_{i}=\exp \left(2 \pi i n_{i} / m_{i}\right)$ and $y_{j k}=\exp \left(2 \pi i k n_{i} /\left(m_{i} l\right)\right), k=1 \ldots(l-1), n_{i}, m_{i} \in \mathbb{N}_{+}$. Let us now consider the cases $\mathbf{w}=1,2$ in more detail.

\section{A. $w=1$}

The first element is real and occurs at $\mathrm{I}=2$,

$$
\operatorname{Li}_{1}\left(e_{2}\right)=-\ln (2)
$$

representing the simplest alternating multiple zeta value, cf., e.g., Ref. 11 . At I= 3 we get the complex conjugate numbers

$$
\begin{aligned}
& \operatorname{Li}_{1}\left(e_{3}\right)=-\frac{1}{2} \ln (3)+\frac{\pi i}{6}, \\
& \operatorname{Li}_{1}\left(e_{3}^{2}\right)=-\frac{1}{2} \ln (3)-\frac{\pi i}{6} .
\end{aligned}
$$


Due to (6.9), $\ln (3)$ and $i \pi$ are considered as basis elements from this level on. For all higher values of I one thus needs only to consider the real part of the $w=1$ sums and one may work with the real representations given in Sec. V. Let us consider the following example:

$$
\begin{aligned}
\operatorname{Li}_{1}\left(e_{4}\right) & =-\ln \left(1-e_{4}\right) \\
& =e_{4} \sum_{k=1}^{\infty} \frac{1}{4 k-3}+e_{2} \sum_{k=1}^{\infty} \frac{1}{4 k-2}+e_{4}^{*} \sum_{k=1}^{\infty} \frac{1}{4 k-1}+e_{1} \sum_{k=1}^{\infty} \frac{1}{4 k} \\
& =e_{4} \sum_{k=1}^{\infty}\left(\frac{1}{4 k-3}-\frac{1}{4 k}\right)+e_{2} \sum_{k=1}^{\infty}\left(\frac{1}{4 k-2}-\frac{1}{4 k}\right)+e_{4}^{*} \sum_{k=1}^{\infty}\left(\frac{1}{4 k-1}-\frac{1}{4 k}\right) .
\end{aligned}
$$

Equation (6.17) follows from

$$
\sum_{k=1}^{n-1} e_{n}^{k}=0 .
$$

The type of sums occurring in (6.17) leads to digamma-functions and one may use their relations given before to find the corresponding basis representations. The first terms are given by

$$
\begin{aligned}
& \operatorname{Li}_{1}\left(e_{4}\right)=-\frac{1}{2} \ln (2)+\frac{\pi i}{4}, \\
& \operatorname{Li}_{1}\left(e_{4}^{3}\right)=\operatorname{Li}_{1}^{*}\left(e_{4}\right), \\
& \mathrm{Li}_{1}\left(e_{5}\right)=\frac{1}{2} \ln \left(\frac{\sqrt{5}+1}{2}\right)-\frac{1}{4} \ln (5)+i \frac{3}{10} \pi, \\
& \operatorname{Li}_{1}\left(e_{5}^{2}\right)=\frac{1}{2} \ln \left(\frac{\sqrt{5}-1}{2}\right)-\frac{1}{4} \ln (5)+i \frac{1}{10} \pi, \\
& \operatorname{Re}\left(\operatorname{Li}_{1}\left(e_{5}\right)\right)+\operatorname{Re}\left(\operatorname{Li}_{1}\left(e_{5}^{2}\right)\right)=-\frac{1}{2} \ln (5) \\
& \operatorname{Li}_{1}\left(e_{6}\right)=\frac{\pi i}{3} \\
& \operatorname{Re}\left(\operatorname{Li}_{1}\left(e_{8}\right)\right)=-\frac{1}{4} \ln (2)-\frac{1}{2} \ln (\sqrt{2}-1), \\
& \operatorname{Re}\left(\operatorname{Li}_{1}\left(e_{12}\right)\right)=\frac{1}{2} \ln (2)-\ln (\sqrt{3}-1) .
\end{aligned}
$$

In Table VI we summarize the number of basis elements.

At cyclotomy I=9 we find one basis element less than that reported in Ref. 82. The new elements contributing at the respective level of cyclotomy for $I \leq 20$ are

$$
\begin{array}{ll}
\mathrm{I}=2 & \ln (2), \\
\mathrm{I}=3 & \ln (3), \pi \\
\mathrm{I}=4 & -
\end{array}
$$


TABLE VI. The number of the basis elements spanning the $\mathrm{w}=1$ cyclotomic harmonic polylogarithms at $l$ th roots of unity up to 20 .

\begin{tabular}{|c|c|c|c|c|c|c|c|c|c|c|c|c|c|c|c|c|c|c|c|c|}
\hline 1 & 1 & 2 & 3 & 4 & 5 & 6 & 7 & 8 & 9 & 10 & 11 & 12 & 13 & 14 & 15 & 16 & 17 & 18 & 19 & 20 \\
\hline Basis & 0 & 1 & 2 & 2 & 3 & 3 & 4 & 3 & 4 & 4 & 6 & 4 & 7 & 5 & 6 & 5 & 9 & 5 & 10 & 6 \\
\hline Ref. 82 & 0 & 1 & 2 & 2 & 3 & 3 & 4 & 3 & 5 & 4 & 6 & & & & & & & & & \\
\hline New & & & & & & & & & & & & & & & & & & & & \\
\hline elements & 0 & 1 & 2 & 0 & 2 & 0 & 3 & 1 & 2 & 0 & 5 & 1 & 6 & 0 & 2 & 2 & 8 & 0 & 9 & 2 \\
\hline
\end{tabular}

$$
\begin{aligned}
& \text { I = } 5 \quad \operatorname{Re}\left(\operatorname{Li}_{1}\left(e_{5}\right)\right), \operatorname{Re}\left(\operatorname{Li}_{1}\left(e_{5}^{2}\right)\right), \\
& \mathrm{I}=6 \quad- \\
& \mathrm{I}=\left.7 \quad \operatorname{Re}\left(\operatorname{Li}_{1}\left(e_{7}^{k}\right)\right)\right|_{k=1} ^{3}, \\
& I=8 \quad \operatorname{Re}\left(\operatorname{Li}_{1}\left(e_{8}\right)\right), \\
& I=9 \quad \operatorname{Re}\left(\operatorname{Li}_{1}\left(e_{9}\right)\right), \operatorname{Re}\left(\operatorname{Li}_{1}\left(e_{9}^{2}\right)\right), \\
& \mathrm{I}=10 \quad- \\
& \mathrm{I}=\left.11 \quad \operatorname{Re}\left(\operatorname{Li}_{1}\left(e_{11}^{k}\right)\right)\right|_{k=1} ^{5}, \\
& \mathrm{I}=12 \quad \operatorname{Re}\left(\operatorname{Li}_{1}\left(e_{12}\right)\right), \\
& \mathrm{I}=\left.13 \quad \operatorname{Re}\left(\operatorname{Li}_{1}\left(e_{13}\right)\right)\right|_{k=1} ^{6}, \\
& \mathrm{I}=14 \quad- \\
& \text { I = } 15 \quad \operatorname{Re}\left(\operatorname{Li}_{1}\left(e_{15}\right)\right), \operatorname{Re}\left(\operatorname{Li}_{1}\left(e_{15}^{2}\right)\right), \\
& \text { I = } 16 \quad \operatorname{Re}\left(\operatorname{Li}_{1}\left(e_{16}\right)\right), \operatorname{Re}\left(\operatorname{Li}_{1}\left(e_{16}^{3}\right)\right), \\
& I=\left.17 \quad \operatorname{Re}\left(\operatorname{Li}_{1}\left(e_{17}\right)\right)\right|_{k=1} ^{8}, \\
& \mathrm{I}=18 \quad- \\
& I=\left.19 \quad \operatorname{Re}\left(\operatorname{Li}_{1}\left(e_{19}\right)\right)\right|_{k=1} ^{9}, \\
& I=20 \quad \operatorname{Re}\left(\operatorname{Li}_{1}\left(e_{20}\right)\right), \operatorname{Re}\left(\operatorname{Li}_{1}\left(e_{20}^{3}\right)\right) .
\end{aligned}
$$


B. $w=2$

We first consider the relations for $\operatorname{Li}_{2}\left(e_{n}^{k}\right)$. The following well-known representations for the function holds, cf. Refs. 83 and 84:

$$
\mathrm{Li}_{2}\left(e^{i \theta}\right)=\pi^{2} \bar{B}_{2}\left(\frac{\theta}{2 \pi}\right)+i \mathrm{Cl}_{2}(\theta)
$$

with

$$
\begin{aligned}
\mathrm{Cl}_{2}(\theta) & =\sum_{k=1}^{\infty} \frac{\sin (k \theta)}{k^{2}}, \\
\bar{B}_{2}(x) & =-\frac{1}{\pi^{2}} \sum_{k=1}^{\infty} \frac{\cos (2 \pi k x)}{k^{2}} .
\end{aligned}
$$

$\bar{B}_{2}$ denotes the second modified Bernoulli polynomial. Due to (6.10) only the imaginary parts have to be considered. The number $\pi$ occurs at $w=1, I=3$ only. The first terms are given by

$$
\begin{aligned}
& \operatorname{Li}_{2}\left(e_{1}\right)=\frac{\pi^{2}}{6} \\
& \operatorname{Li}_{2}\left(e_{2}\right)=-\frac{\pi^{2}}{12} \\
& \operatorname{Im}\left(\operatorname{Li}_{2}\left(e_{3}\right)\right)=\frac{\sqrt{3}}{9}\left[\psi^{(1)}\left(\frac{1}{3}\right)-\frac{2}{3} \pi^{2}\right] \\
& \operatorname{Im}\left(\operatorname{Li}_{2}\left(e_{4}\right)\right)=\mathbf{C}, \\
& \operatorname{Li}_{2}\left(e_{4}^{3}\right)=\mathrm{Li}_{2}^{*}\left(e_{4}\right) \\
& \operatorname{Im}\left(\operatorname{Li}_{2}\left(e_{5}\right)\right)=5 \sqrt{10}\left\{\sqrt{1+\frac{1}{\sqrt{5}}}\left[\psi^{(1)}\left(\frac{1}{5}\right)-\pi^{2}\left(1+\frac{1}{\sqrt{5}}\right)\right]\right. \\
& \left.+\sqrt{1-\frac{1}{\sqrt{5}}}\left[\psi^{(1)}\left(\frac{2}{5}\right)-\pi^{2}\left(1-\frac{1}{\sqrt{5}}\right)\right]\right\} \\
& \operatorname{Im}\left(\operatorname{Li}_{2}\left(e_{5}^{2}\right)\right)=\frac{i}{5 \sqrt{10}}\left\{\sqrt{1-\frac{1}{\sqrt{5}}}\left[\psi^{(1)}\left(\frac{1}{5}\right)-\pi^{2}\left(1+\frac{1}{\sqrt{5}}\right)\right]\right. \\
& \left.-\sqrt{1+\frac{1}{\sqrt{5}}}\left[\psi^{(1)}\left(\frac{2}{5}\right)-\pi^{2}\left(1-\frac{1}{\sqrt{5}}\right)\right]\right\}, \\
& \operatorname{Li}_{2}\left(e_{5}^{3}\right)=\operatorname{Li}_{2}^{*}\left(e_{5}^{2}\right), \\
& \operatorname{Li}_{2}\left(e_{5}^{4}\right)=\operatorname{Li}_{2}^{*}\left(e_{5}\right), \\
& \operatorname{Im}\left(\operatorname{Li}_{2}\left(e_{6}\right)\right)=\frac{3}{2} \operatorname{Im}\left(\operatorname{Li}_{2}\left(e_{3}\right)\right),
\end{aligned}
$$




$$
\begin{aligned}
\operatorname{Li}_{2}\left(e_{6}^{5}\right) & =\mathrm{Li}_{2}^{*}\left(e_{6}\right) \\
\operatorname{ImLi}_{2}\left(e_{8}\right) & =\frac{\sqrt{2}}{32} \psi^{\prime}\left(\frac{1}{8}\right)+\frac{1}{4}(1-2 \sqrt{2}) \mathbf{C}-\frac{1}{16}(1+\sqrt{2}) \pi^{2} \\
\operatorname{ImLi}_{2}\left(e_{12}\right) & =\frac{\sqrt{3}}{24} \psi^{\prime}\left(\frac{1}{3}\right)+\frac{2}{3} \mathrm{C}-\frac{\sqrt{3}}{36} \pi^{2} .
\end{aligned}
$$

The new basis elements spanning the dilogarithms of the $l$ th roots of unity for $I \leq 20$ are

$$
\begin{aligned}
& \mathrm{I}=1,2 \quad- \\
& I=3 \quad \operatorname{Im}\left(\operatorname{Li}_{2}\left(e_{3}\right)\right) \\
& \mathrm{I}=4 \quad \mathrm{C} \\
& I=5 \quad \operatorname{Im}\left(\operatorname{Li}_{2}\left(e_{5}\right)\right), \operatorname{Im}\left(\operatorname{Li}_{2}\left(e_{5}^{2}\right)\right) \\
& I=6 \quad- \\
& \mathrm{I}=\left.7 \quad \operatorname{Im}\left(\mathrm{Li}_{2}\left(e_{7}^{k}\right)\right)\right|_{k=1} ^{3}, \\
& \mathrm{I}=8 \quad \operatorname{Im}\left(\mathrm{Li}_{2}\left(e_{8}\right)\right) \\
& I=9 \quad \operatorname{Im}\left(\operatorname{Li}_{2}\left(e_{9}\right)\right), \operatorname{Im}\left(\operatorname{Li}_{2}\left(e_{9}^{2}\right)\right) \\
& \mathrm{I}=10 \\
& I=\left.11 \quad \operatorname{Im}\left(\operatorname{Li}_{2}\left(e_{11}^{k}\right)\right)\right|_{k=1} ^{5}, \\
& I=12 \quad \operatorname{Im}\left(\operatorname{Li}_{2}\left(e_{12}\right)\right), \\
& I=\left.13 \quad \operatorname{Im}\left(\operatorname{Li}_{2}\left(e_{13}\right)\right)\right|_{k=1} ^{6}, \\
& \mathrm{I}=14 \quad- \\
& I=15 \quad \operatorname{Im}\left(\operatorname{Li}_{2}\left(e_{15}\right)\right),
\end{aligned}
$$




$$
\begin{array}{ll}
\mathrm{I}=16 & \operatorname{Im}\left(\operatorname{Li}_{2}\left(e_{16}\right)\right), \operatorname{Im}\left(\operatorname{Li}_{2}\left(e_{16}^{3}\right)\right), \\
\mathrm{I}=17 & \left.\operatorname{Im}\left(\operatorname{Li}_{2}\left(e_{17}\right)\right)\right|_{k=1} ^{8}, \\
\mathrm{I}=18 & - \\
\mathrm{I}=19 & \left.\operatorname{Im}\left(\operatorname{Li}_{2}\left(e_{19}\right)\right)\right|_{k=1} ^{9}, \\
\mathrm{I}=20 & \operatorname{Im}\left(\operatorname{Li}_{2}\left(e_{20}\right)\right) .
\end{array}
$$

Let us now turn to all convergent sums $\sigma_{1,1}(x, y), x, y \in \mathcal{C}_{n}$. These sums belong to cyclotomy I if $x=e_{l}^{k_{1}}, y=e_{l}^{k_{2}}$, and $k_{1}, k_{2} \in \mathbb{N}_{+}, k_{1}<l, k_{2}<l$.

We consider first the case $x \neq 1, y=1$,

$$
\begin{aligned}
\sigma_{1,1}(x, 1) & =\operatorname{Li}_{2}(x)+\frac{1}{2} \operatorname{Li}_{1}^{2}(x) \\
& =\frac{1}{2} \operatorname{Re}\left(\operatorname{Li}_{1}^{2}(x)\right)+\left(r_{2}-\frac{1}{2} r_{1}^{2}\right) \pi^{2}+i\left[r_{1} \pi \operatorname{Re}\left(\operatorname{Li}_{1}(x)\right)+\operatorname{Im}\left(\operatorname{Li}_{2}(x)\right)\right]
\end{aligned}
$$

with

$$
\begin{aligned}
& r_{1}=\operatorname{Im}(-\ln (1-x)), \\
& r_{2}=\operatorname{Re}\left(\operatorname{Li}_{2}(x)\right) .
\end{aligned}
$$

Including the basis elements of $\mathbf{w}=1$ up to $\mathrm{l}$, no new basis element is obtained. Furthermore,

$$
\begin{gathered}
\sigma_{1,1}\left(e_{2}, x\right)=-\frac{1}{2} \pi^{2}+\frac{1}{2} \ln ^{2}(2)+\mathrm{Li}_{2}\left(\frac{1-x}{2}\right) \\
\sigma_{1,1}\left(x, e_{2}\right)=-\mathrm{Li}_{1}(x) \ln (2)+\frac{1}{2} \operatorname{Li}_{2}\left(x^{2}\right)-\mathrm{Li}_{2}(x)+\frac{1}{2}\left[\pi^{2}+\ln ^{2}(2)\right]-\mathrm{Li}_{2}\left(\frac{1-x}{2}\right)
\end{gathered}
$$

hold. $\mathrm{Li}_{2}((1+x) / 2)$ may yield a new basis element. In some cases besides $x$, also $-x$ is an element of the cycle of the roots of unity which have to be considered. Here, however, $\operatorname{Li}_{2}((1+x) / 2)$ is given by

$$
\operatorname{Li}_{2}\left(\frac{1+x}{2}\right)=-\mathrm{Li}_{2}\left(\frac{1-x}{2}\right)+\frac{\pi^{2}}{6}-\operatorname{Li}_{1}(x) \operatorname{Li}_{1}(-x)-\ln ^{2}(2)-\ln (2)\left[\operatorname{Li}_{1}(x)+\operatorname{Li}_{1}(-x)\right] .
$$

Also, the elements $x=e_{n}^{k}$ and $e_{1}-x \equiv 1-x$ occur in the cycles, for which

$$
\operatorname{Li}_{2}\left(1-e_{n}^{k}\right)=-\operatorname{Li}_{2}\left(e_{n}^{k}\right)+2 \pi i \frac{k}{n} \operatorname{Li}_{1}\left(e_{n}^{k}\right)+\frac{\pi^{2}}{6}
$$

holds.

For $\mathrm{I}=2$, one obtains

$$
\sigma_{1,1}\left(e_{2}, 1\right)=-\frac{1}{2} \pi^{2}+\frac{1}{2} \ln ^{2}(2)
$$


Because of (6.50) two basis elements contribute. If a corresponding special number has occurred already at $w=1$ at the same value of $I$, it is not counted as new. $\pi$ occurs at $w=2, I=1$, unlike for $w=1$ where it contributes first at $I=3$. No new basis element occurs at $I=2$. For $w=3,4$ the new basis elements occur for $\operatorname{Li}_{2}\left(e_{n}^{k}\right)$ only. We apply the relations, cf., e.g., Ref. 64,

$$
\begin{aligned}
& \operatorname{Li}_{2}\left(\frac{1}{1+x}\right)=\mathrm{Li}_{2}(-x)+\ln (x) \ln (1+x)-\frac{1}{2} \ln ^{2}(1+x)+\zeta_{2}, \\
& \mathrm{Li}_{2}\left(\frac{x}{1+x}\right)=-\mathrm{Li}_{2}(-x)-\frac{1}{2} \ln ^{2}(1+x) .
\end{aligned}
$$

At $I=5$ the above relations lead to corresponding reductions and the two functions

$$
\mathrm{Li}_{2}\left(-\frac{e_{5}^{3}\left(1-e_{5}\right)}{1-e_{5}^{3}}\right), \quad \operatorname{Li}_{2}\left(-\frac{e_{5}^{2}}{1+e_{5}}\right)
$$

remain. For the first function the identity

$$
\mathrm{Li}_{2}\left(-\frac{e_{5}^{3}\left(1-e_{5}\right)}{1-e_{5}^{3}}\right)=\mathrm{Li}_{2}\left(\frac{e_{5}^{4}}{1+e_{5}^{4}}\right)=-\mathrm{Li}_{2}\left(-e_{5}^{4}\right)-\frac{1}{2} \ln ^{2}\left(1+e_{5}^{4}\right)
$$

holds, through which the corresponding sum $\sigma_{1,1}\left(e_{5}^{3}, e_{5}\right)$ can be expressed by

$$
\sigma_{1,1}\left(e_{5}^{3}, e_{5}\right)=\frac{1}{2} \operatorname{Li}_{2}^{*}\left(e_{5}^{2}\right)-\operatorname{Li}_{2}^{*}\left(e_{5}\right)-\frac{1}{2}\left(\operatorname{Li}_{1}^{2}\left(e_{5}\right)\right)^{*}+\operatorname{Li}_{1}\left(e_{5}\right)^{*} \operatorname{Li}_{1}\left(e_{5}^{2}\right)^{*} .
$$

The second dilogarithm can be transformed in the following way:

$$
\mathrm{Li}_{2}\left(-\frac{e_{5}^{2}}{1+e_{5}}\right)=-\mathrm{Li}_{2}\left(\frac{1+e_{5}+e_{5}^{2}}{1+e_{5}}\right)-\ln \left(-\frac{e_{5}^{2}}{1+e_{5}}\right) \ln \left(\frac{1+e_{5}+e_{5}^{2}}{1+e_{5}}\right)+\zeta_{2}
$$

Furthermore,

$$
\operatorname{Li}_{2}\left(\frac{1+e_{5}+e_{5}^{2}}{1+e_{5}}\right)=\operatorname{Li}_{2}\left(-e_{5}^{3}\right)
$$

holds, through which

$$
\operatorname{Li}_{2}\left(-\frac{e_{5}^{2}}{1+e_{5}}\right)=-\frac{1}{2} \operatorname{Li}_{2}\left(e_{5}\right)+\operatorname{Li}_{2}^{*}\left(e_{5}^{2}\right)+\frac{19}{150} \pi^{2}-\frac{i \pi}{5}\left[\operatorname{Li}_{1}\left(e_{5}^{2}\right)-\operatorname{Li}_{1}\left(e_{5}\right)\right]
$$

is obtained.

The representations at $w=6$ were given in Ref. 20 , with $\operatorname{Li}_{4}(1 / 2)$ being the new basis element. The Clausen function $\mathrm{Cl}_{2}(\pi / 3)$ used there is given by

$$
\begin{aligned}
& \mathrm{Cl}_{n}(x)=\left\{\begin{array}{ll}
\frac{i}{2}\left[\mathrm{Li}_{n}(\exp (-i x))-\mathrm{Li}_{n}(\exp (i x))\right], & n \text { even } \\
\frac{1}{2}\left[\mathrm{Li}_{n}(\exp (-i x))+\mathrm{Li}_{n}(\exp (i x))\right], & n \text { odd }
\end{array},\right. \\
& \mathrm{Cl}_{2}\left(\frac{\pi}{3}\right)=\mathrm{Ls}_{2}^{(0)}\left(\frac{\pi}{3}\right)=\frac{3}{2} \operatorname{Im}\left(\mathrm{Li}_{2}\left(e_{3}\right)\right)
\end{aligned}
$$

with

$$
\operatorname{Ls}_{j}^{(k)}(\theta)=-\int_{0}^{\theta} d t t^{k} \ln ^{j-k-1}\left|2 \sin \left(\frac{t}{2}\right)\right|,
$$

cf. Ref. 85. In Table VII we summarize the number of basis elements found at $w=2$ using the relations in Sec. IV C, the distribution relations (6.12) and (6.13), and the relations for dilogarithms given above. We also list the number of basis elements for the class of dilogarithms at roots of unity, and in both cases the number of new elements beyond those being obtained at $w=1$ at the same value of $I$. 
TABLE VII. The number of the basis elements spanning the dilogarithms, respectively. $\mathbf{w}=2$ cyclotomic harmonic sums at $l$ th roots of unity up to 20 .

\begin{tabular}{lrrrrrrrrrrrrrrrrrrrr}
\hline \hline \multicolumn{1}{c}{1} & 1 & 2 & 3 & 4 & 5 & 6 & 7 & 8 & 9 & 10 & 11 & 12 & 13 & 14 & 15 & 16 & 17 & 18 & 19 & 20 \\
\hline Li 2 basis & 1 & 1 & 2 & 2 & 3 & 2 & 4 & 3 & 4 & 3 & 6 & 3 & 7 & 4 & 5 & 5 & 9 & 4 & 10 & 5 \\
Li new $_{\text {n }}$ & 1 & 0 & 1 & 1 & 2 & 0 & 3 & 1 & 2 & 0 & 5 & 0 & 6 & 0 & 1 & 2 & 8 & 0 & 9 & 1 \\
Basis & 1 & 2 & 3 & 3 & 5 & 5 & 8 & 7 & 10 & 10 & 16 & 12 & 21 & 17 & 21 & 21 & 33 & 23 & 40 & 29 \\
Ref. 82 & 1 & 1 & 1 & 1 & 2 & 2 & 4 & 4 & 7 & 6 & 10 & & & & & & & & & \\
New & & & & & & & & & & & & & & & & & & & & \\
elements & 1 & 0 & 1 & 1 & 2 & 1 & 4 & 3 & 5 & 4 & 10 & 5 & 14 & 8 & 12 & 12 & 24 & 11 & 30 & 16 \\
\hline \hline
\end{tabular}

At $\mathbf{w}=2$ the respective new basis elements are

$$
\begin{aligned}
& \mathrm{I}=1 \quad \pi, \\
& \mathrm{I}=2 \quad-
\end{aligned}
$$

$$
I=3 \quad \operatorname{Im}\left(\operatorname{Li}_{2}\left(e_{3}\right)\right)
$$

$\mathrm{I}=4 \quad \mathbf{C}$,

$I=5 \quad \operatorname{Im}\left(\operatorname{Li}_{2}\left(e_{5}\right)\right), \operatorname{Im}\left(\operatorname{Li}_{2}\left(e_{5}^{2}\right)\right)$,

$$
I=6 \quad \operatorname{Li}_{4}\left(\frac{1}{2}\right)
$$

$$
\mathrm{I}=\left.7 \quad \operatorname{Im}\left(\operatorname{Li}_{2}\left(e_{7}^{k}\right)\right)\right|_{k=1} ^{3}, \sigma_{1,1}\left(e_{7}, e_{7}^{2}\right)
$$

$$
I=8 \quad \operatorname{Im}\left(\operatorname{Li}_{2}\left(e_{8}\right)\right), \sigma_{1,1}\left(e_{8}, e_{4}\right), \sigma_{1,1}\left(e_{8}, e_{8}^{3}\right),
$$

$$
\mathrm{I}=9 \quad \operatorname{Im}\left(\mathrm{Li}_{2}\left(e_{9}\right)\right), \operatorname{Im}\left(\operatorname{Li}_{2}\left(e_{9}^{2}\right)\right), \sigma_{1,1}\left(e_{9}, e_{9}^{2}\right), \sigma_{1,1}\left(e_{9}, e_{3}\right), \sigma_{1,1}\left(e_{9}^{2}, e_{3}\right),
$$

$$
\begin{aligned}
& \mathrm{I}=10 \quad \sigma_{1,1}\left(e_{5}, e_{2}\right), \sigma_{1,1}\left(e_{5}^{2}, e_{2}\right), \sigma_{1,1}\left(e_{10}, e_{5}\right), \sigma_{1,1}\left(e_{10}, e_{10}^{3}\right), \\
& \mathrm{I}=\left.11 \quad \operatorname{Im}\left(\operatorname{Li}_{2}\left(e_{11}^{k}\right)\right)\right|_{k=1} ^{5},\left.\sigma_{1,1}\left(e_{11}, e_{11}^{k}\right)\right|_{k=2} ^{4},\left.\sigma_{1,1}\left(e_{11}^{2}, e_{11}^{k}\right)\right|_{k=3} ^{4}, \quad \text { etc. }
\end{aligned}
$$

We mention that counting relations for majorants of the motivic numbers, which are claimed to be related to the bases of the sums $\sigma_{k_{1}, \ldots, k_{m}}\left(x_{1}, \ldots, x_{m}\right), x_{j} \in \mathcal{C}_{n}$, were given in Ref. 77. The dimension of the respective $\mathbb{Q}$-vector space is majorized by the expansion coefficients $D_{n}(\mathrm{~W})$ of

$$
G_{\mathrm{w}}(x)=\sum_{n=0}^{\infty} D_{n}(\mathrm{w}) x^{n}
$$


with

$$
\begin{aligned}
G_{1}(x) & =\frac{1}{1-x^{2}-x^{3}}, \\
G_{2}(x) & =\frac{1}{1-x-x^{2}}, \\
G_{\mathrm{k}}(x) & =\frac{1}{1-\frac{1}{2}[\varphi(k)+v] x-(v-1) x^{2}}, k \geq 3 .
\end{aligned}
$$

Here, $\varphi(k)$ denotes Euler's totient function ${ }^{24}$ and $v$ is the number of prime factors of $k$.

\section{CONCLUSIONS}

In evaluating massive 3-loop integrals with local operator insertions nested sums occur, containing denominators which belong to the class of harmonic sums generated by cyclotomic polynomials. To simplify the computations, the relations between these quantities have to be known and used in computer algebra codes, such as SIGMA (Ref. 17) and HARMONICSUMS (Ref. 27) to allow for an efficient reduction in the corresponding summation problem. In integrals of similar structure we expect even more general terms (1.5) to occur.

The usual harmonic sums ${ }^{7,8}$ and polylogarithms ${ }^{26}$ were thus generalized to cover the newly occurring structures. We started with the harmonic polylogarithms extending the usual alphabet of denominators ranging to $\Phi_{2}(x)$ to general cyclotomic polynomials $\Phi_{n}(x), n \geq 3$. The cyclotomic harmonic polylogarithms form a shuffle algebra. They have support $x \in[0,1]$ and one may define a Mellin transform, usually of argument $k N$, with $k, N \in \mathbb{N}_{+}$which spans the finite cyclotomic harmonic sums (2.1) together with special values as the cyclotomic harmonic sums in the limit $N \rightarrow \infty$.

The cyclotomic harmonic sums are meromorphic functions with poles at the non-positive integers. They obey recurrence relations in terms of sums of lower depth and one may derive analytical asymptotic representations. In this way the cyclotomic harmonic sums are analytically continued from integer values of the sum index $N$ to $N \in \mathbb{C}$. The cyclotomic harmonic sums form a quasi-shuffle algebra, cf. Ref. 9. After the analytic continuation they obey differentiation relations, accounting for their values at $N \rightarrow \infty$. Furthermore, three multiple argument relations apply. Using these relations one may reduce the number of cyclotomic harmonic sums vastly growing with the weight to smaller bases. We study the case of the sums following from iteration of the denominators (4.60) up to weight $\mathbf{w}=5$. Corresponding counting relations for the number of basis elements are obtained.

The values of the cyclotomic harmonic sums for $N \rightarrow \infty$, respectively, the values of the cyclotomic harmonic polylogarithms at $x=1$, are of special interest. They are linearly related to the infinite nested harmonic sums with roots of unity as numerator weight factors. Already in case of the single infinite cyclotomic harmonic sums a large number of new constants contribute beyond those known in the case of multiple zeta values and Euler-Zagier values. ${ }^{11}$ The quasi-shuffle and multiple argument relations of the cyclotomic harmonic sums and the shuffle relations of the cyclotomic harmonic polylogarithms allow to derive basis representations induced by these relations. We studied in this respect the case of the infinite cyclotomic harmonic sums based on the iteration of the summands (4.60) to weight $\mathrm{W}=6$ and the sums of weight $\mathrm{W}=1,2$ for cyclotomy $\mathrm{I} \leq 20$. Using computer algebra methods the explicit representation of all infinite cyclotomic harmonic sums was derived as well. For wide classes of relations explicit counting relations of the basis elements were given. The corresponding representations were derived with the MATHEMATICA-based computer algebra system HARMONICSUMS. ${ }^{27}$

The present investigations can be readily extended to cyclotomic harmonic sums and polylogarithms of higher weight and cyclotomy, both in the case of finite values of $N$ and for $N \rightarrow \infty$, using the present methods. The requested computational time and storage resources grow accordingly. This applies, in particular, to the derivation of the explicit representations of all sums over the corresponding bases. 
We finally considered also the generalized harmonic sums of weight $\mathbf{w}=1,2$ at $l$ th roots of unity for $1 \leq l \leq 20$. They obey dilogarithmic representations. Besides the shuffle and stuffle relations, they obey distribution relations and the known relations for dilogarithms. We used these relations to derive corresponding basis representations. Compared to the case of the infinite cyclotomic harmonic sums these sums obey more symmetries. Thus, at a given weight and cyclotomy $l$ they are represented by a lower number of basis elements. We compared to results in literature.

\section{ACKNOWLEDGMENTS}

We thank D. Broadhurst for providing us relations (5.137) and (5.138). For discussions we would like to thank D. Broadhurst, F. Brown, and D. Kreimer, and N.J.A. Sloan and M. Kauers for remarks on special issues. We would like to thank H. Kawamura for Ref. 54. This work has been supported in part by the Deutsche Forschungsgemeinschaft (DFG) Sonderforschungsbereich Transregio 9, Computergestützte Theoretische Teilchenphysik, by the Austrian Science Fund (FWF) Grant Nos. P20162-N18 and P203477-N18, and by the European Union (EU) Network LHCPHENOnet PITN-GA-2010-264564.

\section{APPENDIX A: SOME PROPERTIES OF CYCLOTOMIC POLYNOMIALS}

In the following we summarize some technical aspects needed to represent expressions used in the present paper.

First, we summarize some aspects on cyclotomic polynomials. ${ }^{23}$ We give the decompositions of the polynomials

$$
x^{l}+1, l \in \mathbb{N} \backslash\{0\}
$$

in terms of cyclotomic polynomials for $l \leq 20$ :

$$
\begin{aligned}
& x^{3}+1=\Phi_{2}(x) \Phi_{6}(x), \\
& x^{5}+1=\Phi_{2}(x) \Phi_{10}(x), \\
& x^{6}+1=\Phi_{4}(x) \Phi_{12}(x), \\
& x^{7}+1=\Phi_{2}(x) \Phi_{14}(x), \\
& x^{9}+1=\Phi_{2}(x) \Phi_{6}(x) \Phi_{18}(x), \\
& x^{10}+1=\Phi_{4}(x) \Phi_{20}(x), \\
& x^{14}+1=\Phi_{4}(x) \Phi_{28}(x), 1=\Phi_{2}(x) \Phi_{22}(x), \\
& x^{13}+1=\Phi_{2}(x) \Phi_{26}(x), \\
& x^{12}+1=\Phi_{8}(x) \Phi_{24}(x),
\end{aligned}
$$




$$
\begin{aligned}
& x^{15}+1=\Phi_{2}(x) \Phi_{6}(x) \Phi_{10}(x) \Phi_{30}(x), \\
& x^{17}+1=\Phi_{2}(x) \Phi_{34}(x), \\
& x^{18}+1=\Phi_{4}(x) \Phi_{12}(x) \Phi_{36}(x), \\
& x^{19}+1=\Phi_{2}(x) \Phi_{39}(x), \\
& x^{20}+1=\Phi_{8}(x) \Phi_{40}(x) .
\end{aligned}
$$

For odd values of $n$,

$$
\Phi_{2 n}(x)=\Phi_{n}(-x)
$$

holds. The decomposition

$$
x^{2 k+1}-1=(x-1) \prod_{i} \Phi_{i}(x)
$$

results thus into

$$
x^{2 k+1}+1=\Phi_{2}(x) \prod_{i} \Phi_{2 i}(x) .
$$

From

$$
\Phi_{2 n}(x) \mid\left(x^{2 n}-1\right) \quad \text { and } \quad \Phi_{2 n}(x) \quad \chi\left(x^{n}-1\right),
$$

it follows

$$
\Phi_{2 n}(x) \mid\left(x^{n}+1\right) \text {. }
$$

If $p$ is a prime and $p \mid n$ then $^{86}$

$$
\Phi_{p n}(x)=\Phi_{n}\left(x^{p}\right) .
$$

For $n=2^{k}, k \in \mathbb{N}_{+}$, it follows that all $\Phi_{2^{k}}(x)$ are cyclotomic polynomials. In (A6), (A12), and (A14) more factors than just $\Phi_{2 n}(x)$ occur. They originate due to power-rescaling, i.e.,

$$
\begin{aligned}
& \frac{x^{15}+1}{x^{3}+1}=\frac{y^{5}+1}{y+1}=\Phi_{10}(y), \\
& \frac{x^{15}+1}{x^{5}+1}=\frac{y^{3}+1}{y+1}=\Phi_{6}(y) .
\end{aligned}
$$

Therefore, all the factors of $\left(x^{5}+1\right)$ and $\left(x^{3}+1\right)$ have to emerge in the decomposition, and similarly for other $N$ with more non-trivial divisors. For $N=2^{k} \cdot n$ where the integer $n>1$ is odd, we get

$$
\frac{x^{2^{k} \cdot n}+1}{x^{2^{k}}+1}=\frac{y^{n}+1}{y+1}=\Phi_{2 n}(y) .
$$

The argument remains valid if $n$ is a product of odd primes. Therefore, the only cyclotomic polynomials of the structure $x^{a}+1$ are those with $a=2^{k}, k \in \mathbb{N}_{+}$.

For a proper definition of the cyclotomic harmonic polynomials which appear in sum representations like (2.6)

$$
\frac{1}{x^{l} \pm 1}, l \in \mathbb{N}_{+}
$$


we perform partial fractioning. In the following we provide the corresponding expressions in terms of the words $f_{k}^{l}(x)$ forming the cyclotomic harmonic polylogarithms up to $l=6$ :

$$
\begin{aligned}
& (x-1)^{-1}=f_{1}^{0}(x), \\
& (x+1)^{-1}=f_{2}^{0}(x), \\
& \left(x^{2}-1\right)^{-1}=\frac{1}{2}\left[f_{1}^{0}(x)-f_{2}^{0}(x)\right], \\
& \left(x^{2}+1\right)^{-1}=f_{4}^{0}(x), \\
& \left(x^{3}-1\right)^{-1}=\frac{1}{3}\left[f_{1}^{0}(x)-2 f_{3}^{1}(x)-f_{3}^{0}(x)\right], \\
& \left(x^{3}+1\right)^{-1}=\frac{1}{3}\left[f_{2}^{0}(x)-f_{6}^{1}(x)+2 f_{6}^{0}(x)\right], \\
& \left(x^{4}-1\right)^{-1}=\frac{1}{4}\left[f_{1}^{0}(x)-f_{2}^{0}(x)-2 f_{4}^{0}(x)\right], \\
& \left(x^{4}+1\right)^{-1}=f_{8}^{0}(x) \\
& \left(x^{5}-1\right)^{-1}=\frac{1}{5}\left[f_{1}^{0}(x)-\frac{4}{5} f_{5}^{0}(x)-\frac{3}{5} f_{5}^{1}(x)-\frac{2}{5} f_{5}^{2}(x)-\frac{1}{5} f_{5}^{3}(x)\right], \\
& \left(x^{5}+1\right)^{-1}=\frac{1}{5}\left[f_{2}^{0}(x)+\frac{4}{5} f_{5}^{0}(x)-\frac{3}{5} f_{5}^{1}(x)+\frac{2}{5} f_{5}^{2}(x)-\frac{1}{5} f_{5}^{3}(x)\right], \\
& \left(x^{6}-1\right)^{-1}=\frac{1}{6}\left[f_{1}^{0}(x)-f_{2}^{0}(x)-2 f_{3}^{0}(x)-f_{3}^{1}(x)-2 f_{6}^{0}(x)+f_{6}^{1}(x)\right], \\
& \left(x^{6}+1\right)^{-1}=\frac{1}{3}\left[f_{4}^{0}(x)+2 f_{12}^{0}(x)-f_{12}^{2}(x)\right], \quad \text { etc. }
\end{aligned}
$$




\section{APPENDIX B: PROOFS OF SOME RELATIONS IN SECTION IV}

\section{Proof of Eq. (4.58)}

We proceed by induction on $m$. Let $m=1$ :

$$
\begin{aligned}
S_{\left\{a_{1}, b_{1}, c_{1}\right\}}(2 n)+S_{\left\{a_{1}, b_{1},-c_{1}\right\}}(2 n)= & \sum_{i=1}^{2 n} \frac{1}{\left(a_{1} i+b_{1}\right)^{c_{1}}}+\sum_{i=1}^{2 n} \frac{(-1)^{i}}{\left(a_{1} i+b_{1}\right)^{c_{1}}} \\
= & \sum_{i=1}^{n}\left(\frac{1}{\left(a_{1} 2 i+b_{1}\right)^{c_{1}}}+\frac{1}{\left(a_{1}(2 i-1)+b_{1}\right)^{c_{1}}}\right) \\
& +\sum_{i=1}^{n}\left(\frac{1}{\left(a_{1} 2 i+b_{1}\right)^{c_{1}}}-\frac{1}{\left(a_{1}(2 i-1)+b_{1}\right)^{c_{1}}}\right) \\
= & 2 \sum_{i=1}^{n} \frac{1}{\left(2 a_{1} i+b_{1}\right)^{c_{1}}}=2 S_{\left\{2 a_{1}, b_{1}, c_{1}\right\}}(n) .
\end{aligned}
$$

In the following we use the abbreviation:

$$
A(n):=\sum S_{\left\{a_{m}, b_{m}, \pm c_{m}\right\}, \ldots,\left\{a_{1}, b_{1}, \pm c_{1}\right\}}(n) .
$$

Now, we assume that (4.58) holds for $m$ :

$$
\begin{aligned}
& \sum S_{\left\{a_{m+1}, b_{m+1}, \pm c_{m+1}\right\}, \ldots,\left\{a_{1}, b_{1}, \pm c_{1}\right\}}(2 n) \\
& =\sum_{i=1}^{2 n} \frac{1}{\left(a_{m+1} i+b_{m+1}\right)^{c_{m+1}}} \sum S_{\left\{a_{m}, b_{m}, \pm c_{m}\right\}, \ldots,\left\{a_{1}, b_{1}, \pm c_{1}\right\}}(i) \\
& +\sum_{i=1}^{2 n} \frac{(-1)^{i}}{\left(a_{m+1} i+b_{m+1}\right)^{c_{m+1}}} \sum S_{\left\{a_{m}, b_{m}, \pm c_{m}\right\}, \ldots,\left\{a_{1}, b_{1}, \pm c_{1}\right\}}(i) \\
& =\sum_{i=1}^{2 n} \frac{A(i)}{\left(a_{m+1} i+b_{m+1}\right)^{c_{m+1}}}+\sum_{i=1}^{2 n} \frac{(-1)^{i} A(i)}{\left(a_{m+1} i+b_{m+1}\right)^{c_{m+1}}} \\
& =\sum_{i=1}^{n}\left(\frac{A(2 i)}{\left(2 a_{m+1} i+b_{m+1}\right)^{c_{m+1}}}+\frac{A(2 i-1)}{\left((2 i-1) a_{m+1}+b_{m+1}\right)^{c_{m+1}}}\right) \\
& +\sum_{i=1}^{n}\left(\frac{(-1)^{2 i} A(2 i)}{\left(2 a_{m+1} i+b_{m+1}\right)^{c_{m+1}}}+\frac{(-1)^{2 i-1} A(2 i-1)}{\left((2 i-1) a_{m+1}+b_{m+1}\right)^{c_{m+1}}}\right) \\
& =2 \sum_{i=1}^{n} \frac{A(2 i)}{\left(2 a_{m+1} i+b_{m+1}\right)^{c_{m+1}}} \\
& =2 \sum_{i=1}^{n} \frac{1}{\left(2 a_{m+1} i+b_{m+1}\right)^{c_{m+1}}} 2^{m} S_{\left\{2 a_{m}, b_{m}, c_{m}\right\}, \ldots,\left\{2 a_{1}, b_{1}, c_{1}\right\}}(i) \\
& =2^{m+1} S_{\left\{2 a_{m+1}, b_{m+1}, c_{m+1}\right\}, \ldots,\left\{2 a_{1}, b_{1}, c_{1}\right\}}(n) \text {. }
\end{aligned}
$$




\section{Proof of Eq. (4.59)}

We proceed by induction on $m$. Let $m=1$ :

$$
\begin{aligned}
S_{\left\{a_{1}, b_{1}, c_{1}\right\}}(2 n)-S_{\left\{a_{1}, b_{1},-c_{1}\right\}}(2 n)= & \sum_{i=1}^{2 n} \frac{1}{\left(a_{1} i+b_{1}\right)^{c_{1}}}+\sum_{i=1}^{2 n} \frac{(-1)^{i}}{\left(a_{1} i+b_{1}\right)^{c_{1}}} \\
= & \sum_{i=1}^{n}\left(\frac{1}{\left(a_{1} 2 i+b_{1}\right)^{c_{1}}}+\frac{1}{\left(a_{1}(2 i-1)+b_{1}\right)^{c_{1}}}\right) \\
& -\sum_{i=1}^{n}\left(\frac{1}{\left(a_{1} 2 i+b_{1}\right)^{c_{1}}}-\frac{1}{\left(a_{1}(2 i-1)+b_{1}\right)^{c_{1}}}\right) \\
= & 2 \sum_{i=1}^{n} \frac{1}{\left((2 i-1) a_{1}+b_{1}\right)^{c_{1}}}=2 S_{\left\{2 a_{1}, b_{1}-a_{1}, c_{1}\right\}}(n) .
\end{aligned}
$$

In the following we use the abbreviation

$$
A(n):=\sum d_{m} \cdots d_{1} S_{\left\{a_{m}, b_{m}, d_{m} c_{m}\right\}, \ldots,\left\{a_{1}, b_{1}, d_{1} c_{1}\right\}}(n) .
$$

Note that

$$
\begin{aligned}
A(2 n-1)= & A(2 n)-\sum d_{m} \cdots d_{1} \frac{d_{m}^{2 n} S_{\left\{a_{m-1}, b_{m-1}, d_{m-1} c_{m-1}\right\}, \ldots,\left\{a_{1}, b_{1}, d_{1} c_{1}\right\}}(2 n)}{\left(a_{m} 2 n+b_{m}\right)^{c_{m}}} \\
= & A(2 n)-\sum d_{m-1} \cdots d_{1} \frac{S_{\left\{a_{m-1}, b_{m-1}, d_{m-1} c_{m-1}\right\}, \ldots,\left\{a_{1}, b_{1}, d_{1} c_{1}\right\}}(2 n)}{\left(a_{m} 2 n+b_{m}\right)^{c_{m}}} \\
& +\sum d_{m-1} \cdots d_{1} \frac{S_{\left\{a_{m-1}, b_{m-1}, d_{m-1} c_{m-1}\right\}, \ldots,\left\{a_{1}, b_{1}, d_{1} c_{1}\right\}}(2 n)}{\left(a_{m} 2 n+b_{m}\right)^{c_{m}}}=A(2 n) .
\end{aligned}
$$

Now, we assume that (4.59) holds for $m$ :

$$
\begin{aligned}
\sum d_{m+1} & \cdots d_{1} S_{\left\{a_{m+1}, b_{m+1}, d_{m+1} c_{m+1}\right\}, \ldots,\left\{a_{1}, b_{1}, d_{1} c_{1}\right\}}(2 n) \\
= & \sum_{i=1}^{2 n} \frac{1}{\left(a_{m+1} i+b_{m+1}\right)^{c_{m+1}}} \sum d_{m} \cdots d_{1} S_{\left\{a_{m}, b_{m}, d_{m} c_{m}\right\}, \ldots,\left\{a_{1}, b_{1}, d_{1} c_{1}\right\}}(i) \\
& -\sum_{i=1}^{2 n} \frac{(-1)^{i}}{\left(a_{m+1} i+b_{m+1}\right)^{c_{m+1}}} \sum d_{m} \cdots d_{1} S_{\left\{a_{m}, b_{m}, d_{m} c_{m}\right\}, \ldots,\left\{a_{1}, b_{1}, d_{1} c_{1}\right\}}(i) \\
= & \sum_{i=1}^{2 n} \frac{A(i)}{\left(a_{m+1} i+b_{m+1}\right)^{c_{m+1}}}-\sum_{i=1}^{2 n} \frac{(-1)^{i} A(i)}{\left(a_{m+1} i+b_{m+1}\right)^{c_{m+1}}} \\
= & \sum_{i=1}^{n}\left(\frac{A(2 i)}{\left(2 a_{m+1} i+b_{m+1}\right)^{c_{m+1}}}+\frac{A(2 i-1)}{\left((2 i-1) a_{m+1}+b_{m+1}\right)^{c_{m+1}}}\right) \\
& -\sum_{i=1}^{n}\left(\frac{(-1)^{2 i} A(2 i)}{\left(2 a_{m+1} i+b_{m+1}\right)^{c_{m+1}}}+\frac{(-1)^{2 i-1} A(2 i-1)}{\left((2 i-1) a_{m+1}+b_{m+1}\right)^{c_{m+1}}}\right)
\end{aligned}
$$




$$
\begin{aligned}
& =2 \sum_{i=1}^{n} \frac{A(2 i-1)}{\left((2 i-1) a_{m+1}+b_{m+1}\right)^{c_{m+1}}}=2 \sum_{i=1}^{n} \frac{A(2 i)}{\left((2 i-1) a_{m+1}+b_{m+1}\right)^{c_{m+1}}} \\
& =2 \sum_{i=1}^{n} \frac{1}{\left(2 a_{m+1} i+b_{m+1}-a_{m+1}\right)^{c_{m+1}}} 2^{m} S_{\left\{2 a_{m}, b_{m}-a_{m}, c_{m}\right\}, \ldots,\left\{2 a_{1}, b_{1}-a_{1}, c_{1}\right\}}(n) \\
& =2^{m+1} S_{\left\{2 a_{m+1}, b_{m+1}-a_{m+1}, c_{m+1}\right\}, \ldots,\left\{2 a_{1}, b_{1}-a_{1}, c_{1}\right\}}(n) .
\end{aligned}
$$

\section{Proof of Eqs. (4.82) to (4.86)}

We start with $C_{f_{0}^{0}, \vec{m}}(x)$. We consider integrals of the form

$$
\begin{aligned}
\int_{0}^{x} \frac{1}{y} \sum_{i=1}^{\infty} \frac{\sigma^{i} y^{2 i+c_{j}}}{\left(2 i+c_{j}\right)^{a}} S_{\boldsymbol{n}}(i) d y & =\sum_{i=1}^{\infty} \frac{\sigma^{i}}{\left(2 i+c_{j}\right)^{a}} S_{\boldsymbol{n}}(i) \int_{0}^{x} y^{2 i+c_{j}-1} d y \\
& =\sum_{i=1}^{\infty} \frac{\sigma^{i}}{\left(2 i+c_{j}\right)^{a}} S_{\boldsymbol{n}}(i) \frac{x^{2 i+c_{j}}}{2 i+c_{j}} d y \\
& =\sum_{i=1}^{\infty} \frac{\sigma^{i} x^{2 i+c_{j}}}{\left(2 i+c_{j}\right)^{a+1}} S_{\boldsymbol{n}}(i) .
\end{aligned}
$$

Summing over $j$ yields the desired result.

For $C_{f_{4}^{0}, \vec{m}}(x)$ we consider the following integrals:

$$
\begin{aligned}
\int_{0}^{x} \frac{1}{1+y^{2}} \sum_{i=1}^{\infty} \frac{\sigma^{i} y^{2 i+c_{j}}}{\left(2 i+c_{j}\right)^{a}} S_{\boldsymbol{n}}(i) d y & =\int_{0}^{x} \sum_{i=1}^{\infty}(-1)^{i} y^{2 i} \sum_{i=0}^{\infty} \frac{\sigma^{i+1} y^{2 i+c_{j}+2}}{\left(2 i+c_{j}+2\right)^{a}} S_{\boldsymbol{n}}(i+1) d y \\
& =\int_{0}^{x} \sum_{i=1}^{\infty} \sum_{k=0}^{i}(-1)^{i-k} y^{2 i-2 k} \frac{\sigma^{k+1} y^{2 k+c_{j}+2}}{\left(2 k+c_{j}+2\right)^{a}} S_{\boldsymbol{n}}(k+1) d y \\
& =\int_{0}^{x} \sum_{i=1}^{\infty}(-1)^{i+1} y^{2 i+c_{j}+2} \sum_{k=0}^{i} \frac{(-\sigma)^{k+1}}{\left(2 k+c_{j}+2\right)^{a}} S_{\boldsymbol{n}}(k+1) d y \\
& =\sum_{i=1}^{\infty} \frac{(-1)^{i+1} y^{2 i+c_{j}+3}}{2 i+c_{j}+3} \sum_{k=1}^{i+1} \frac{(-\sigma)^{k}}{\left(2 k+c_{j}\right)^{a}} S_{\boldsymbol{n}}(k) \\
& =\sum_{i=1}^{\infty} \frac{(-1)^{i} x^{2 i+c_{j}+1}}{\left(2 i+c_{j}+1\right)} S_{\left\{2, c_{j},-\sigma a\right\}, \boldsymbol{n}}(i) .
\end{aligned}
$$

Summing over $j$ yields the desired result. The case $C_{f_{4}^{1}, \vec{m}}(x)$ follows analogously.

For $C_{f_{2}^{0}, \vec{m}}(x)$ we consider the integrals of the form

$$
\begin{aligned}
\int_{0}^{x} \frac{1}{1+y} \sum_{i=1}^{\infty} \frac{\sigma^{i} y^{2 i+c_{j}}}{\left(2 i+c_{j}\right)^{a}} S_{n}(i) d y & =\int_{0}^{x} \frac{1-y}{1-y^{2}} \sum_{i=1}^{\infty} \frac{\sigma^{i} y^{2 i+c_{j}}}{\left(2 i+c_{j}\right)^{a}} S_{\boldsymbol{n}}(i) d y \\
& =\int_{0}^{x} \frac{1}{1-y^{2}} \sum_{i=1}^{\infty} \frac{\sigma^{i} y^{2 i+c_{j}}}{\left(2 i+c_{j}\right)^{a}} S_{n}(i) d y
\end{aligned}
$$




$$
\begin{aligned}
& -\int_{0}^{x} \frac{y}{1-y^{2}} \sum_{i=1}^{\infty} \frac{\sigma^{i} y^{2 i+c_{j}}}{\left(2 i+c_{j}\right)^{a}} S_{n}(i) d y \\
& =\int_{0}^{x} \sum_{i=0}^{\infty} y^{2 i} \sum_{i=0}^{\infty} \frac{\sigma^{i+1} y^{2 i+c_{j}+2}}{\left(2 i+c_{j}+2\right)^{a}} S_{\boldsymbol{n}}(i+1) d y \\
& -\int_{0}^{x} \sum_{i=0}^{\infty} y^{2 i+1} \sum_{i=0}^{\infty} \frac{\sigma^{i+1} y^{2 i+c_{j}+2}}{\left(2 i+c_{j}+2\right)^{a}} S_{\boldsymbol{n}}(i+1) d y \\
& =\int_{0}^{x} \sum_{i=0}^{\infty} \sum_{k=0}^{i} y^{2 i-2 k} \frac{\sigma^{k+1} y^{2 k+c_{j}+2}}{\left(2 k+c_{j}+2\right)^{a}} S_{\boldsymbol{n}}(k+1) d y \\
& -\int_{0}^{x} \sum_{i=0}^{\infty} \sum_{k=0}^{i} y^{2 i-2 k+1} \frac{\sigma^{k+1} y^{2 k+c_{j}+2}}{\left(2 k+c_{j}+2\right)^{a}} S_{\boldsymbol{n}}(k+1) d y \\
& =\int_{0}^{x} \sum_{i=0}^{\infty} y^{2 i+c_{j}+1} S_{\left\{2, c_{j}, \sigma a\right\}, \boldsymbol{n}}(i+1) d y \\
& -\int_{0}^{x} \sum_{i=0}^{\infty} y^{2 i+c_{j}+3} S_{\left\{2, c_{j}, \sigma a\right\}, \boldsymbol{n}}(i+1) d y \\
& =\sum_{i=1}^{\infty} \frac{x^{2 i+c_{j}+1}}{\left(2 i+c_{j}+1\right)} S_{\left\{2, c_{j}, \sigma a\right\}, \boldsymbol{n}}(i) \\
& -\sum_{i=1}^{\infty} \frac{x^{2 i+c_{j}+2}}{\left(2 i+c_{j}+2\right)} S_{\left\{2, c_{j}, \sigma a\right\}, \boldsymbol{n}}(i) .
\end{aligned}
$$

Summing over $j$ yields the desired result. The case $C_{f_{1}^{0}, \vec{m}}(x)$ follows analogously.

${ }^{1}$ G. 't Hooft and M. J. G. Veltman, Nucl. Phys. B 44, 189 (1972).

${ }^{2}$ G. 't Hooft and M. J. G. Veltman, Nucl. Phys. B 153, 365 (1979); Diagrammar, NATO Adv. Study Inst. Ser., Ser. B 4, 177 (1974); G. Passarino and M. J. G. Veltman, Nucl. Phys. B 160, 151 (1979).

${ }^{3}$ P. Appell, Sur Les Fonctions Hypérgéometriques de Plusieurs Variables (Gauthier-Villars, Paris, 1925); P. Appell and J. Kampé de Fériet, Fonctions Hypérgéometriques; Polynômes d'Hermite (Gauthier-Villars, Paris, 1926); W. N. Bailey, Generalized Hypergeometric Series (Cambridge University Press, Cambridge, England, 1935); Higher Transcendental Functions, Bateman Manuscript Project Vol. I, edited by A. Erdélyi (McGraw-Hill, New York, 1953); L. J. Slater, Generalized Hypergeometric Functions (Cambridge University Press, Cambridge, England, 1966); H. Exton, Multiple Hypergeometric Functions and Applications (Ellis Horwood Limited, Chichester, 1976); H. Exton, Handbook of Hypergeometric Integrals (Ellis Horwood Limited, Chichester, 1978).

${ }^{4}$ C. G. Bollini and J. J. Giambiagi, Nuovo Cimento B 12, 20 (1972); J. F. Ashmore, ibid. 4, 289 (1972); G. M. Cicuta and E. Montaldi, ibid. 4, 329 (1972).

${ }^{5}$ A. M. Legendre, Mém. Inst. Fr. 10, 416 (1809); S. D. Poisson, Mém. Inst. Fr. 257, 57 (1811); C. F. Gauss, Comment. Gotting. Bd. 2, 34 (1812).

${ }^{6}$ J. Blümlein, Comput. Phys. Commun. 180, 2218 (2009); e-print [arXiv:0901.3106 [hep-ph]].

${ }^{7}$ J. Blümlein and S. Kurth, Phys. Rev. D 60, 014018 (1999); e-print [arXiv:hep-ph/9810241].

${ }^{8}$ J. A. M. Vermaseren, Int. J. Mod. Phys. A 14, 2037 (1999); e-print [arXiv:hep-ph/9806280].

${ }^{9}$ M. E. Hoffman, J. Algebra 194, 477 (1997); Nucl. Phys. B (Proc. Suppl.) 135, 215 (2004); e-print [arXiv:math/0406589].

${ }^{10}$ L. Euler, Novi Commentarii academiae scientiarum imperialis Petropolitanae (1775), Vol. 20, p. 140, reprinted in Opera Omnia Ser I, (B.G. Teubner, Berlin, 1927), Vol. 15, p. 217; D. Zagier, in First European Congress of Mathematics (Paris, 1992), Vol. II; Prog. Math. 120, 497 (Birkhäuseere, Basel, 1994).

${ }^{11}$ D. J. Broadhurst, e-print [hep-th/9604128]; D. J. Broadhurst and D. Kreimer, Phys. Lett. B 393, 403 (1997); e-print [hep-th/9609128]; J. M. Borwein, D. M. Bradley, D. J. Broadhurst, and P. Lisonek, Trans. Am. Math. Soc. 353, 907 (2001); e-print [arXiv:math/9910045]; J. Blümlein, D. J. Broadhurst, and J. A. M. Vermaseren, Comput. Phys. Commun. 181, 582 (2010); e-print [arXiv:0907.2557 [math-ph]], and references therein.

12 J. A. M. Vermaseren, A. Vogt, and S. Moch, Nucl. Phys. B 724, 3 (2005); e-print [arXiv:hep-ph/0504242].

${ }^{13}$ S. Moch, P. Uwer, and S. Weinzierl, J. Math. Phys. 43, 3363 (2002); e-print [hep-ph/0110083].

${ }^{14}$ J. Ablinger, J. Blümlein, and C. Schneider, (unpublished). 
${ }^{15}$ J. Ablinger, I. Bierenbaum, J. Blümlein, A. Hasselhuhn, S. Klein, C. Schneider, and F. Wissbrock, Nucl. Phys. B (Proc. Suppl.) 205-206, 242 (2010); e-print [arXiv:1007.0375 [hep-ph]].

${ }^{16}$ J. Ablinger, J. Blümlein, S. Klein, C. Schneider, and F. Wissbrock, Nucl. Phys. B 844, 26 (2011); e-print [arXiv:1008.3347 [hep-ph]].

${ }^{17}$ C. Schneider, J. Symb. Comput. 43, 611 (2008); e-print [arXiv:0808.2543v1]; Ann. Comb. 9, 75 (2005); J. Differ. Equations 11, 799 (2005); Ann. Comb. 14(4), 533 (2010); e-print [arXiv:0808.2596]; in Proceedings of the Conference on Motives, Quantum Field Theory, and Pseudodifferential Operators, Clay Mathematics Institute, Boston University, Cambridge, MA, 2-13 June, 2008; Clay Mathematics Proceedings, edited by A. Carey, D. Ellwood, S. Paycha, and S. Rosenberg (Clay Mathematical Institute, Boston, MA, 2010), Vol. 12, p. 285; Sém. Lothar. Combin. 56, 1 (2007), Article B56b, Habilitationsschrift JKU Linz (2007), and references therein; J. Ablinger, J. Blümlein, S. Klein, and C. Schneider, Nucl. Phys. B (Proc. Suppl.)B , 110 (2010); e-print [arXiv:1006.4797 [math-ph]].

${ }^{18}$ See, e.g., J. Zhao, e-print arXiv:0707.1459 [mathNT]; Acad. Sci., Paris, C. R. 346, 1029 (2008); e-print [arXiv:0810.1064[mathNT]]; Doc. Math. 15, 1 (2010); J. Algebra Appl. 9, 327 (2010); e-print [arXiv:0904.0888[mathNT]]; [cf. also footnote 8 of Ref. 29].

${ }^{19}$ A. B. Goncharov, Math. Res. Lett. 5, 497 (1998).

${ }^{20}$ D. J. Broadhurst, Eur. Phys. J. C 8, 311 (1999); e-print [arXiv:hep-th/9803091].

${ }^{21}$ M. Bigotte, G. Jacob, N. E. Oussous, and M. Petitot, Theor. Comput. Sci 273, 271 (2002).

${ }^{22}$ S. Weinzierl, J. Math. Phys. 452656 (2004); e-print [hep-ph/0402131]; M. Y. Kalmykov, B. F. L. Ward, and S. Yost, J. High Energy Phys. 0702, 040 (2007); e-print [hep-th/0612240]; M. Y. Kalmykov, J. High Energy Phys. 0604, 056 (2006); e-print [hep-th/0602028]; M. Y. Kalmykov, B. F. L. Ward, and S. A. Yost, J. High Energy Phys. 0710, 048 (2007); e-print [arXiv:0707.3654 [hep-th]]; T. Huber and D. Maitre, Comput. Phys. Commun. 178, 755 (2008); e-print [arXiv:0708.2443 [hep-ph]]; M. Y. Kalmykov and B. A. Kniehl, Nucl. Phys. B 809, 365 (2009); e-print [arXiv:0807.0567 [hep-th]].

${ }^{23}$ S. Lang, Algebra, 3rd ed. (Springer, New York, 2002).

${ }^{24}$ L. Euler, Novi Commentarii academiae scientiarum imperialis Petropolitanae (1760), Vol. 8, p. 74; M. Takase, "Euler's Theory of Numbers," in Euler Reconsidered, edited by R. Baker (Kedrick, Heber City, UT, 2007), p. 377; see leonhardeuler.web.fc2.com/eulernumber_en.pdf.

${ }^{25}$ H. Poincaré, Acta Math. 4, 201 (1884); J. A. Lappo-Danilevsky, Mémoirs sur la Théorie des Systèmes Différentielles Linéaires (Chelsea, New York, 1953); K. T. Chen, Trans. A.M.S. 156(3), 359 (1971).

${ }^{26}$ E. Remiddi and J. A. M. Vermaseren, Int. J. Mod. Phys. A 15, 725 (2000); e-print [arXiv:hep-ph/9905237].

${ }^{27} \mathrm{~J}$. Ablinger, code harmonicsums (unpublished).

${ }^{28}$ J. Blümlein, Comput. Phys. Commun. 159, 19 (2004); e-print [arXiv:hep-ph/0311046].

${ }^{29}$ J. Blümlein, S. Klein, C. Schneider, and F. Stan, e-print arXiv:1011.2656 [cs.SC].

${ }^{30}$ J. Blümlein, "Structural Relations of Harmonic Sums and Mellin Transforms at Weight w=6," in Proceedings of the Conference on Motives, Quantum Field Theory, and Pseudodifferential Operators, Clay Mathematics Institute, Boston University, Cambridge, MA, June 2-13, 2008; Clay Mathematics Proceedings edited by A. Carey, D. Ellwood, S. Paycha, and S. Rosenberg (2010), Vol. 12, p. 167; e-print [arXiv:0901.0837 [math-ph]].

${ }^{31} \mathrm{~J}$. Ablinger, J. Blümlein, and C. Schneider (unpublished).

${ }^{32}$ J. Blümlein, Comput. Phys. Commun. 133, 76 (2000); e-print [arXiv:hep-ph/0003100]; S. I. Alekhin and J. Blümlein, Phys. Lett. B 594, 299 (2004); e-print [arXiv:hep-ph/0404034]; J. Blümlein and S. O. Moch, Phys. Lett. B 614, 53 (2005); e-print [arXiv:hep-ph/0503188]; A. V. Kotikov and V. N. Velizhanin, e-print [hep-ph/0501274]; S. Albino, Phys. Lett. B 674, 41 (2009); e-print [arXiv:0902.2148 [hep-ph]].

${ }^{33}$ T. Gehrmann and E. Remiddi, Comput. Phys. Commun. 141, 296 (2001); e-print [arXiv:hep-ph/0107173].

${ }^{34}$ J. Vollinga and S. Weinzierl, Comput. Phys. Commun. 167, 177 (2005); e-print [arXiv:hep-ph/0410259].

${ }^{35}$ T. Gehrmann and E. Remiddi, Comput. Phys. Commun. 144, 200 (2002); e-print [arXiv:hep-ph/0111255]; R. Bonciani, G. Degrassi, and A. Vicini, e-print arXiv:1007.1891 [hep-ph].

${ }^{36}$ N. Nielsen, Handbuch der Theorie der Gammafunktion (Teubner, Leipzig, 1906); reprinted by (Chelsea, Bronx, New York, 1965).

${ }^{37} \mathrm{~J}$. Stirling, Methodus differentialis sive tractatus de summatione et interpolatione serierum infinitarum (Impensis Ric. Manby, London, 1730), p. 27.

${ }^{38}$ K. G. Chetyrkin and M. Steinhauser, Nucl. Phys. B 573, 617 (2000); e-print [hep-ph/9911434].

${ }^{39}$ A. I. Davydychev and M. Y. Kalmykov, Nucl. Phys. B 605, 266 (2001); e-print [hep-th/0012189].

${ }^{40}$ L. G. Almeida and C. Sturm, Phys. Rev. D82, 054017 (2010); e-print [arXiv:1004.4613 [hep-ph]]; J. A. Gracey, e-print arXiv:1104.5382 [hep-ph].

${ }^{41}$ C. Reutenauer, Free Lie Algebras (Calendron, Oxford, 1993).

${ }^{42}$ E. Witt, J. Reine Angew. Math. 177, 152 (1937); Math. Zeitschr. 64, 195 (1956).

${ }^{43}$ A. F. Möbius, J. Reine Angew. Math. 9, 105 (1832); G. W. Hardy and E. M. Wright, An Introduction to the Theory of Numbers, 5th ed. (Calendron, Oxford, 1979).

${ }^{44}$ E. Catalan, Mem. Acad. Imp. Sci. Saint-Pétersbourg, Ser. 7, 31 (1883); V. Adamchik, see http://www-2.cs.cmu.edu/ adamchik/articles/catalan/catalan.htm for Catalan numbers.

${ }^{45}$ A. Migotti, Sitzungsber Math.-Naturwiss. Kl Kaiser. Akad. Wiss. Wien 87, 7 (1883); P. Erdös, Bull. Am. Math. Soc. 52, 179 (1946); M. Endo, Comment. Math. Univ. St. Pauli 23, 121 (1974); R. Thangadurai, "On the coefficients of cyclotomic polynomials," in Cyclotomic Fields and Related Topics (Bhaskaracharya, Pune, 2000), p. 311.

${ }^{46}$ E. Landau, Sitzungsber. Math.-Naturw. Kl. Bayerische Akad. Wiss. München 36, 151 (1906).

${ }^{47}$ C. F. Gauss, Comment. Gotting. 2, 33 (1812); J. L. W. V. Jensen, Nyt Tidsskr Math. 2B, 33 (1891).

${ }^{48}$ C. F. Gauss, Discquisitiones Arithmeticae (Leipzig/Gerhard Fleischer, 1801), pp. 365, 366; É. Galois, (uvre mathématiques publiées en 1846 dans le Journal de Liouville), Tome XI 381-444 (1846). 
${ }^{49}$ See http://mathworld.wolfram.com/TrigonometryAnglesPi5.html and similar pages for trigonometric functions of cyclotomic angles.

${ }^{50}$ Ch. Hermite, Acad. Sci., Paris, C. R. 77, 18 (1873); F. Lindemann, Math. Ann. 20, 213 (1882); K. Weierstrass, Sitzungsber. K. Preuss. Akad. Wiss. 2, 1067 (1885); A. Baker, Transcendental Number Theory (Cambridge University Press, Cambridge, England, 1975), and references therein.

${ }^{51}$ H. M. Srivastave and J. Choi, Series Associated with the Zeta and Related Functions (Kluwer Academic Publishers, Dordrecht, 2001).

${ }^{52}$ A. Hurwitz, Z Math. Phys. 27, 86 (1882); T. M. Apostol, Introduction to Analytic Number Theory (Springer, Berlin, 1976), Chap. 12.

${ }^{53}$ M. W. Coffey, J. Math. Phys. 49, 043510 (2008); D. Cvijovic, J. Math. Phys. 50, 023515 (2009).

${ }_{55}^{54}$ S. Takakazu, Katsuyo Sampo (Syotsudo, Edo, 1712).

${ }^{55} \mathrm{~J}$. Bernoulli, Ars conjectandi, opus posthumum. Accedit Tractatus de seriebus infinitis, et epistola gallicé scripta de ludo pilae reticularis (Brüder Thurneysen, Basel, 1713).

${ }^{56}$ J. Miller and V. S. Adamchik, J. Comput. Appl. Math. 100, 201 (1998).

${ }^{57}$ K. S. Kölbig, J. Comput. Appl. Math. 75, 43 (1996).

${ }^{58}$ L. Saalschütz, Vorlesungen über die Bernoullischen Zahlen (Springer, Berlin, 1893).

${ }^{59}$ N. Nielsen, Traité élémentaire des nombres de Bernoulli (Gauthier-Villars, Paris, 1923).

${ }^{60} \mathrm{~L}$. Euler, Institutiones calculi differentialis cum eius usu in analysi finitorum ac doctrina serierum II (Typographeo Petro Galeatii, Ticini, 1787), Chap. VIII, pp. 224, 226, 1st issue (Academiae Imperialis Scientiarum, St. Petersburg, 1755).

${ }^{61}$ H. F. Scherk, "Erste Abhandlung - Betrifft die Bernoullischen und die Eulerschen Zahlen," in Mathematische Abhandlungen (G. Reimer, Berlin, 1825).

${ }^{62}$ M. Lerch, Enseign. Math. 5, 450 (1903); A. Laurinčikas and R. Garunkštis, The Lerch Zeta-function (Kluwer Academic Publishers, Dordrecht, 2010), and references therein.

${ }^{63}$ W. Spence, An Essay on Logarithmic Transcendents (John Murray, London and Archibald Constable and Company, Edinburgh, 1809).

${ }^{64}$ L. Lewin, Dilogarithms and Associated Functions (Macdonald, London, 1958); Polylogarithms and Associated Functions (North-Holland, New York, 1981); D. Zagier, in Frontiers in Number Theory, Physics, and Geometry II: On Conformal Field Theories, Discrete Groups and Renormalization, edited by P. Cartier et al. (Springer, Berlin, 2006), Vol. 2, p. 3.

${ }^{65}$ R. Clausen, J. Reine Angew. Math. 8, 298 (1832).

${ }^{66}$ D. Cvijović and J. Klinkowski, Math. Comput. 68, 1623 (1999); D. Cvijović, J. Math. Anal. Appl. 332, 1056 (2007).

${ }^{67}$ A. M. Legendre, Exercices de calcul intégral, tome 1, 247 (1811).

${ }^{68}$ See Ref. 60, p. 499 (edition of 1755) and Ref. 59.

${ }^{69}$ B. C. Berndt, Ramanujan's Notebooks, Part I (Springer, Berlin, 1985).

${ }^{70}$ R. Sitaramachandrarao, J. Number Theory 25, 1 (1987).

${ }^{71}$ N. Nielsen, "Der Eulersche Dilogarithmus und seine Verallgemeinerungen," in Nova Acta Leopoldina, (Halle, 1909), Vol. XC, Nr. 3; K. S. Kölbig, SIAM J. Math. Anal. 16, 1232 (1986).

${ }^{72}$ P. F. Jordan, Bull. Am. Math. Soc. 79, 681 (1973).

${ }^{73}$ M. S. Milgram, e-print arXiv:math/0406338v2.

${ }^{74}$ O. M. Ogreid and P. Osland, J. Comput. Appl. Math. 98, 245 (1998); e-print [hep-th/9801168]; M. W. Coffey, J. Comput. Appl. Math. 183, 84 (2005); e-print [math-ph/0505051].

${ }^{75}$ B. J. Laurenzi, e-print arXiv:1010.6229 [math-ph].

${ }^{76}$ P. Deligne, Publ. Math., Inst. Hautes Etud. Sci. 112, 101-141 (2010); See http://www.math.ias.edu/people/faculty/deligne/ preprints.

${ }^{77} \mathrm{P}$. Deligne and A. B. Gocharov, Groupes fondamentaux motivique de Tate mixte, IAS preprint, see http://www.math.ias.edu/people/faculty/deligne/preprints.

${ }^{78}$ N. J. A. Sloane, The On-Line Encyclopedia of Integer Sequences; see http://oeis.org/.

${ }^{79}$ H. R. P. Ferguson and D. H. Bailey, RNR Technical Report RNR-91-032, July 14, 1992.

${ }^{80}$ F. Vieta, Opera mathematica, 1579; reprinted (Officina Bonaventurae \& Abrahami Elzeviriorum, Leiden, 1646).

${ }^{81}$ H. Lugowski and J. Weinert, Grundzüge der Algebra (Teubner, Leipzig, 1960), Vol. III.

${ }^{82}$ G. Racinet, C. R. Math. Acad. Sci. 333(1), 5 (2001); e-print [arXiv:math.QA/0012024]

${ }^{83}$ F. W. Newman, Cambridge Dublin Math. J. II, 77-100 (1847); II, 172-191 (1847).

${ }^{84}$ A. N. Kirillov, Prog. Theor. Phys. Suppl. 118, 61 (1995); e-print [hep-th/9408113].

${ }^{85}$ M. Y. Kalmykov and B. A. Kniehl, Nucl. Phys. Proc. Suppl. 205-206, 129 (2010); e-print [arXiv:1007.2373 [math-ph]].

${ }^{86}$ T. Nagell, Introduction to Number Theory (Wiley, New York, 1951).

${ }^{87}$ Complex representations are related to the so-called colored harmonic sums $S_{b, \vec{a}}(p, \vec{r} ; N)=\sum_{k=1}^{N} \frac{p^{k}}{k^{b}} S_{\vec{a}}(\vec{r} ; k)$ with $b, a_{i} \in$ $\mathbb{N}_{+}, p, r_{i} \in \cup_{l=2}^{M}\{\exp [2 \pi i(n / l)], n \in\{1, \ldots, l-1\}\}$, cf. Refs. 18 and 19.

${ }^{88}$ Note that we defined here the second letter by $1 /(x-1)$ which differs in sign from the corresponding letter in Ref. ${ }^{26}$ Numerical implementations were given in Refs. 33 and 34. A few extensions of iterated integrals introduced in Ref. 26 based on linear denominator functions of different kind, which are used in quantum-field theoretic calculations, were made in Refs. 34 and 35.

${ }^{89}$ For the relations given in this section we mostly present the results, giving for a few cases the proofs in Appendix B. The other proofs proceed in a similar manner.

${ }^{90}$ See Ref. 49 for special values of the trigonometric functions occurring in (5.6).

${ }^{91}$ Special examples were also considered in Ref. 53.

${ }^{92}$ We corrected typos in Eq. (10) of Ref. 66.

${ }^{93}$ For similar sums see Ref. ${ }^{74}$.

${ }^{94}$ Relations between colored nested infinite harmonic sums have been investigated also in Refs. 76 and 77 recently.

${ }^{95}$ We would like to thank D. Broadhurst for communicating this relation to us. 